\title{
Summary of Gamma Spectrometry on Local Air Samples from 1985-1995
}

by

W. G. Winn

Westinghouse Savannah River Company

Savannah River Site

Aiken, South Carolina 29808

This paper was prepared in connection with work done under the above contract number with the U. S. Department of Energy. By acceptance of this paper, the publisher and/or recipient acknowledges the U.S. Government's right to retain a nonexclusive, royalty-free license in and to any copyright covering this paper, along with the right to reproduce and to authorize others to reproduce all or part of the copyrighted paper.<smiles>CC1CCC(C)C1</smiles> 


\section{DISCLAMIER}

Portions of this document may be illegible in electronic image products. Images are produced from the best available original document. 


\section{DISCLAIMER}

This report was prepared as an account of work sponsored by an agency of the United States Government. Neither the United States Government nor any agency thereof, nor any of their employees, makes any warranty, express or implied, or assumes any legal liability or -responsibility for the accuracy, completeness, or usefillness of any information, apparatus, product, or process disclosed, or represents that its use would not infringe privately owned rights. Reference herein to any specific commercial product, process, or service by trade name, trademark, manufacturer, or otherwise does not necessarily constitute or imply its endorsement, recommendation, or favoring by the United States Government or any agency thereof. The views and opinions of authors expressed herein do not necessarily state or reflect those of the United States Government or any agency thereof.

This report has been reproduced directly from the best available copy.

Available to DOE and DOE contractors from the Office of Scientific and Technical Information, P.O. Box 62, Oak Ridge, TN 3̦7831; prices available from (615) 57.6-8401.

Available to the public from the. National Technical-Information Service, U.S. Department of Commerce, 5285 Port Royal Road, Springfield, VA 22161. 
WESTINGHOUSE SAVANNAH RIVER SITE

WSRC-TR-97-0098

SAVANNAH RIVER TECHNOLOGY CENTER

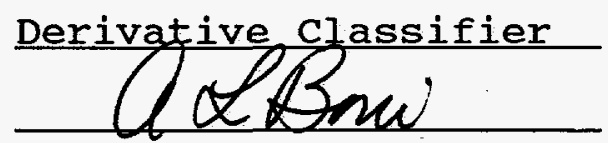

CC: WSRC/SRTC P.T. Deason, 773-A

W.A. Emel, 735-A

R.P. Addis, 773-A

D.W. Hayes, 735-A

J.R. Cadieux, 735-A

D.C. Carlson, 735-A

K.J. Hofstetter, 735-A

K.W. MacMurdo, 735-A

S.H. Reboul, 735-A

D.M. Beals, 735-A

W.H. Carlton, 773-A

G.T. Jannik, 773-A

A.L. Blancett, 773-A

P.A. Cloessner, 773-A

R.C. Hochel, 773-41A

R.A. Sigg, 773-41A

J.B. Gladden, 773-42A
WSRC/EPD M.D. Dukes, 742-A
WSRC/EMS J.D. Heffner, 735-16A
R. Lorenz, 735-16
R.H. Young, 735-16A

DOE W.J. Brumley, 703-47A

T.J. Spears, 703-46A

W.A. Richardson, 223-5H

T.L. Williams, 703-42A

E.C. Goodson, 703-46A

TIS File copies (4)

TO: A.L. BONI, 773-A

April 2, 1997

J.E. HALVERSON, 735-A

FROM: W.G. WINN, 735-A $/ \mathcal{H} /$

Summary of Gamma spectrometry on Local Air samples From 1985 to 1995 (U)

(Cover Sheet) 
WESTINGHOUSE SAVANNAH RIVER SITE SAVANNAH RIVER TECHNOLOGY CENTER

WSRC-TR-97-0098

CC: Distribution on Cover sheet

April 2, 1997

TO: A.L. BONI, 773-A

J.E. HALVERSON, 735-A

FROM: W.G. WINN, 735-A

\section{Summary of Gamma spectrometry on Local Air samples} From 1985 to 1995 (U)

\section{INTRODUCTION}

This report summarizes the 1985-1995 results of low-level HPGe gamma spectrometry analysis of high-volume air samples collected at the Aiken Airport, which is about 25 miles north of SRS as shown in Figure 1. The author began analyzing these samples with new calibrations using the newly developed GRABGAM code $^{1}$ in 1985. The air sample collections were terminated in 1995, as the facilities at the Aiken Airport were no longer available. Air sample measurements prior to 1985 were conducted with a different analysis system (and by others prior to 1984), and the data were not readily available.

The report serves to closeout this phase of local NTS air sample studies, while documenting the capabilities and accomplishments. Hopefully, the information will guide other applications for this technology, both locally and elsewhere. 


\section{MEASUREMENTS}

The air sampler system is shown in Figure 2, and one of its 20 "x20" cellulose collection filters is shown in Figure 3 . Typically about $100,000 \mathrm{~m}^{3}$ of air was passed through such a filter during a 3-day period, after which the filter was removed and bagged into a triangular geometry (6"-sides, 8.5"-hypotenuse,

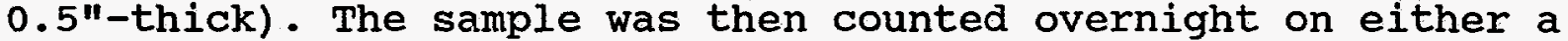
$10 \%$ or $30 \%$ HPGe detector, which had been calibrated for this geometry using source mapping techniques. The detectors were well-shielded within the ULLCF; however, current NTS capabilities with the larger $90 \%$ and $160 \%$ HPGe detectors of the Underground counting Facility offer much better sensitivity.

\section{MEASUREMENTS DATA}

Air sample isotopic data for gamma-emitting radionuclides are presented in Figures 4-16, where selected isotope intensities are plotted against time. The selected isotopes were either cosmogenic or man-made; however, not all of the chernobyl isotopes are included as they have been reported in detail elsewhere 2,3 .

The numerical values for time plots are given in Appendix B, which is saved as a LOTUS spreadsheet file. Except for Cs-137, only values that are above the detection limit are recorded. For Cs-137, "less-than" detection Iimits are denoted as negative values. Entries with only one significant digit are rough estimates for marginal detection. Other entries were detected with counting errors that were better than the determination limit of $\pm 33 \%$.

The original GRABGAM analysis data are archived in ordered notebooks, should anyone wish to study other information associated with the measurements. A gap in the data occurred in September-October of 1988, where repairs to the sampler were apparently being conducted.

COMMENTS - General Phenomena and Events

Both naturally and artificially produced isotopes are reported and discussed in detail in the next section. Atmospheric and solar correlations are considered for the naturally produced isotopes of $\mathrm{Be}-7$ and $\mathrm{Na}-22$. The artificially produced isotopes are discussed in relation to events at SRS and other nuclear sites, although not all observations could be correlated.

Eleven notable events concerning the observed man-made radionuclides and their correlation status were tabulated to guide the reader. Some correlations were identified using 
information from the annual SRS environmental reports ${ }^{4}$. Only two notable events occurred following 1987, after SRS nuclear production activities had been significantly reduced. The tabulation is given below:

Event-Date

A. $09 / 03-09 / 04 / 85$

B. $01 / 31-02 / 03 / 86$

C. $05 / 10-05 / 11 / 86$

D. $11 / 05-11 / 07 / 86$

E. $01 / 26-01 / 28 / 87$

F. $06 / 05-06 / 08 / 87$

G. $07 / 13-07 / 15 / 87$

H. $08 / 19-08 / 21 / 87$

I. $11 / 23-11 / 25 / 87$

J. $08 / 22-08 / 24 / 90$

K. $04 / 21-04 / 23 / 93$
Correlation

no

yes

yes

yes

no

no

no

no

yes

no

yes

\section{Description}

Maximum $\mathrm{Nb}-95, \mathrm{Zr}-95$

Releases from $A$ and $H$ areas

Chernobyl fallout maximum

$\mathrm{H}$ area $\mathrm{Ce}-144$ and 6 other isotopes

Cs-137 spike

Maximum Co-60 with Cs-137 spike

Ce-141 spike and Co-60; no Cs-137

Cs-137 spike

$\mathrm{H}$ area release per leaky valve

Ce-144 spike

Tomsk fallout observation per $\mathrm{Ru}-106$

\section{COMMENTS - Reported Isotopes}

Be-7 (53d). Be-7 is plotted in Figure 4 (raw data) and Figure 5 (smoothed data). The overall geometric average for the $\mathrm{Be}-7$ concentration was $131.5 \mathrm{fCi} / \mathrm{m}^{3}$, which is in good agreement with the EMS average 25 miles from SRS during this period ${ }^{4}$. The Be-7 levels appear anti-correlated with the 11-yr sunspot cycle, where a general dip in the data during 1990-1991 corresponds to the sunspot maximum. Jim Cadieux noted that this anti-correlation holds for C-14 also. Jim also noted fine structure oscillations that appear to peak near the equinoxes, which Dave Hayes noted as probable meteorological effects. Al Boni commented that a pronounced upper atmospheric dumping to the troposphere in Spring may cause some Be-7 peaking, and cliff Carlson noted a similar possibility for Fall.

Na-22 (2.6y). Na-22 is plotted in Figure 6. It is detected by its $1274 \mathrm{keV}$ gamma-ray. Although Eu-154 also has a $1274 \mathrm{keV}$ gamma, other Eu-154 gammas should have been detected; thus, the possibility of Eu-154 was discarded. Al Boni indicated that Na-22 does turn up in air samples from natural sources. He also noted that the data tend to show more $\mathrm{Na}-22$ observations in spring, consistent with upper atmosphere dumping to the troposphere. A possible cosmogenic origin is from the F-19 $(\alpha, n) \mathrm{Na}-22$ reaction. $\mathrm{Na}-22$ was detected in only $15.1 \%$ of the air samples, yielding a geometric average of $0.0224 \mathrm{fCi} / \mathrm{m}^{3}$. Assuming the distribution of Na-22 concentrations to be log-normal implies that the $15.1 \%$ observed values all exceed a value of $\mu+1.03 \sigma$, where $\mu$ is the true log-mean $\log (v)$ of the distribution. Using, a method developed in Appendix A yields $\mathrm{v}=0.0054 \mathrm{fCi} / \mathrm{m}^{3}$, which is significantly lower than the mean of the observed values. 
Co-60 (5.3y). Co-60 is plotted in Figure 7 , where its detection appears to have been sporadic over the years. Co-60 was only observed in $1.43 \%$ of the measurements, which ranged from a barely detected $0.01 \mathrm{fCi} / \mathrm{m}^{3}$ to $0.14 \mathrm{fCi} / \mathrm{m}^{3}$. No particular events have been correlated with the observations; however, the detection of both Co-60 gammas for much of the data strongly confirms its presence. Maximum Co-60 occurred for the 6/5-8/87 event, which also had elevated cs-137 at $0.78 \mathrm{fCi} / \mathrm{m}^{3}$.

$\mathrm{Nb}-95$ (35d). $\mathrm{Nb}-95$ is plotted in Figure 8; it normally occurs with Zr-95 as a fission product. Both were detected in the Chernobyl fallout; however, the largest detection was earlier in 1985, although no incident has been associated with the observation. For these larger $\mathrm{Nb}-95$ concentrations, Al Boni suggested that there may be a correlation with earlier Cf-252 activities at the site. Maximum $\mathrm{Nb}-95$ occurred in an early sample (9/3-4/85); other notable occurrences were with Chernobyl (5/10$11 / 86)$ and the high Ce-144 observation (11/5-7/86). For many of the smaller detections, Nb-95 was detected without Zr-95 (or vice versa); it is possible that these smaller occurrences were actually from high fluctuations in peaks of $\mathrm{Bi}-214$ (or Ac-228 for $\mathrm{Zr}-95)$, as these were observed in only about $3 \%$ of the samples (1\% for $\mathrm{Zr}-95$ ), while Bi-214 (and AC-228) were observed nearly $100 \%$ of the time.

Zr-95 (64d). Zr-95 is plotted in Figure 9; its features are described within the Nb-95 discussion above.

Ru-103 (39d). Ru-103 is plotted in Figure 10; it was mainly observed in the Chernobyl fallout (5/10-11/86). It was also among the 6 man-made isotopes accompanying the high Ce-144 event (11/5$7 / 86)$, although at levels too low to be observed in Figure 10 . The Ru-103 from Chernobyl fallout continued to be observed beyond the time frame covered in the earlier chernobyl reports ${ }^{2-3}$.

Ru-106 (362d): Ru-106 is plotted in Figure 11; it was observed in both the Chernobyl fallout (5/11-11/86) and Tomsk fallout (4/21-23/93). Ru-106 was also among the 6 man-made isotopes accompanying the high ce-144 event (11/5-7/86). The Chernobyl peak activity was $29.2 \mathrm{fCi} / \mathrm{m}^{3}$ in May of 1986 and the Tomsk fallout was barely detected at $0.13 \mathrm{fCi} / \mathrm{m}^{3}$ in April of 1993.

Confirmation of the Tomsk observation resulted from recounting samples on the $90 \%$ HPGe of the Underground counting Facility, whereby $12 \%$ counting statistics were observed for Ru106. Independent observation of $\mathrm{Ru}-106$ at EML in New York provided further confirmation. Unlike the Chernobyl reactor fallout, the Tomsk reprocessing facility fallout did not include the shorter-lived Ru-103. Dave Hayes speculates that the absence 
of other longer-lived fission products in the TOMSK fallout is due to the nature of the accident, whereby favorable oxidation of $\mathrm{Ru}$ causes it to be more readily airborne.

cs-134 (2.1Y). Cs-134 is plotted in Figure 12; it was mainly observed in the chernobyl fallout (5/10-11/86). It was also among the 6 man-made isotopes accompanying the high Ce-144 event (11/57/86), although at levels too low to be distinguished in Figure 12. Following Chernobyl, the $\mathrm{Cs}-134 / \mathrm{Cs}-137$ ratio gradually decreased from 0.5 to less than 0.1 , as pre-existing long-lived Cs-137 on the ground was resuspended and mixed in with the airborne Chernobyl fallout ${ }^{3}$. However, the Cs-134/Cs-137 ratio was 0.23 for the high Ce-144 event, which occurred six months after the peak of the Chernobyl fallout.

Cs-137 (30y) . Cs-137 is plotted in Figures 13 and 14, where Figure 13 has the larger scale to emphasize the chernobyl fallout and Figure 14 uses a smaller scale to illustrate non-Chernobyl levels. Both figures plot MDA "less than" values as negative values. Some relatively high cs-137 activity "spikes" were observed up to more than a year after Chernobyl (through 1987); however, these were generally less than $1 \%$ of the peak chernobyl activity.

Other than Chernobyl, the largest Cs-137 spike was correlated with an' $H$ Area release due to a leaky valve (11/23$25 / 87)$. For this spike and two smaller preceding ones $(1 / 26-28 / 87$ and 8/19-21/87), Cs-137 was the only observed man-made isotope, suggesting the possibility of a common source, namely the leaky valve.

Additional Cs-137 spikes occurred with $A$ and $H$ area releases (1/31-2/3/86), the high Ce-144 event (11/5-7/86), and the maximum Co-60 observation (6/5-8/87). A small Cs-137 spike also occurred with the $\mathrm{Nb}-95 / \mathrm{Zr}-95$ maximum (9/3-4/85).

Ce-141 (33d). Ce-141 is plotted in Figure 15. Like Cs-137, the major ce-141 activity occurred with chernobyl, with two smaller "spikes" being observed about a year later in 1987. One Ce-141 spike (7/13-15/87) was preceded and followed with a little Co-60, while the other ce-141 spike was barely detected.

Ce-144 (284d). Ce-144 is plotted in Figure 16. Ce-144 was observed with Chernobyl, and the only other observations were a spike near the end of 1986 and one in 1990. The larger spike (11/5-7/86) exceed the Ce-144 maximum for the chernobyl fallout and was accompanied by six of the isotopes discussed above, namely $\mathrm{Nb}-95, \mathrm{Zr}-95, \mathrm{Ru}-103, \mathrm{Ru}-106, \mathrm{Cs}-134$, and Cs-137. EMS also observed high Ce-144 (62 fCi/m $\mathrm{m}^{3}$ at $\mathrm{H}$ area during November 1986, but a more precise source correlation was not investigated since the level was within legal limits ${ }^{4}$. The other spike $(8 / 22-24 / 90)$ was relatively small, and its source has not been postulated. 


\section{COMMENTS - Unreported Isotopes}

This report does not provide detailed data for all of the isotopes that were detected. In particular, previously reported isotopes $^{2,3}$ that were observed only during the main Chernobyl fallout are excluded. Also natural isotopes associated with dust and radon are not given. Groupings of these isotopes are given below, should there be future interest in collating their data.

Isotopes only observed during main Chernobyl fallout:

$$
\begin{aligned}
& \text { Ag-110m (33d) } \\
& \text { Sb-125 (2.7y) } \\
& \text { Te-129m (33d) } \\
& \text { Te-132 (3d) } \\
& I-131 \quad(8 d) \\
& C s-136 \quad(13 d) \\
& B a-140 \quad(12 d) \\
& \text { La-140 (12d) }
\end{aligned}
$$

Natural isotopes from dust and radon:

$$
\begin{array}{ll}
\mathrm{K}-40 & \left(1.3 \times 10^{9} \mathrm{Y}\right) \\
\mathrm{Tl}-208 & (3 \mathrm{~m}+) \\
\mathrm{Pb}-210 & (22 \mathrm{y}) \\
\mathrm{Bi}-212 & (61 \mathrm{~m}+) \\
\mathrm{Pb}-212 & (11 \mathrm{~h}+) \\
\mathrm{Bi}-214 & (20 \mathrm{~m}+) \\
\mathrm{Pb}-214 & (27 \mathrm{~m}+) \\
\mathrm{Ra}-226 & (1600 \mathrm{y}) \\
\mathrm{Ac}-228 \quad(6.1 \mathrm{~h}+) \\
\mathrm{Pa}-234 \mathrm{~m} \quad(1.2 \mathrm{~m}+) \\
\mathrm{Th}-234 \quad(24 \mathrm{~d}+) \\
\mathrm{U}-235 \quad\left(7.0 \times 10^{8} \mathrm{Y}\right)
\end{array}
$$

Dave Hayes commented that the natural isotopes may have interesting meteorological correlations; however, one must use the results of the natural isotopes with care. Evidence suggested that a sizeable $K-40$ background existed within the filter, so that $\mathrm{K}-40$ levels are misrepresentative and should not be trusted. Many of the thorium and radium daughters have short half-lives but are denoted by a "+" to indicate that their chain equilibrium half-lives are much longer; probably the equilibrium half-lives exceed $1000 \mathrm{yr}$, as it is unlikely that much $\mathrm{Rn}-222$ (3.9d) was collected by the filter as a precursor, although Rn-222 could be present in the room air background. The infrequently detected Pa$234 \mathrm{~m}$ may be from room background fluctuations, and U-235 detections are most likely $\mathrm{Ra}-226$, as both are denoted by detection of the $186 \mathrm{keV}$ gamma and other less intense U-235 gammas were not observed. 
One should also be cautioned that some of the gammas from these natural sources are similar to those of various man-made radioisotopes. Among these are $\mathrm{Mn}-54, \mathrm{Nb}-95$ and $\mathrm{Zr}-95$. Finally, there were a few marginal detections that the GRABGAM code denoted as Cs-139; however, the associated $166 \mathrm{keV}$ gamma peak was somewhat broad in these cases, possibly suggesting background fluctuations from backscatter.

\section{ACKNOWLEDGEMENTS}

Interpretation: Assistance in data interpretation from Jim Cadieux, Dave Hayes, $\mathrm{Al}$ Boni, and Cliff Carlson have already been acknowledged in the text. Bob Taylor and cliff Carlson provided general information with data interpretations via their earlier oversight of these air collections.

Counting: Filter counting in the ULLCF was coordinated by worth Dalton for a number of years; assisting him were John Thomas, Jim Rushton, Andy Cheek, and Sandy stewart. Beginning in $1993 \mathrm{Jim}$ Cadieux coordinated ULLCF counting with assistance from by susie MacMurray and Jackie Medlin.

Collections: Various outside teams have conducted the field collections over the years. Participants included Richard Penix, clovis Summer, Jerxy Shuford, Raymond Roseberry, and Brian Antonicelli. 


\section{REFERENCES}

1. W.G. Winn, W.W. Bowman, A.L. Boni, "Ultra-Clean Underground Counting Facility for Low-Level Environmental Samples", DP1747 (1987).

2. M.V. Kantelo and W.G. Winn, "Summary of Chernobyl Fallout Monitoring by the Environmental Radiometrics Group", DPST86-624 (1986).

3. W.G. Winn and M.V. Kantelo, "Time-Dependent study of Chernobyl Fallout near Savannah River Site", Trans. Am. Nuc. Soc. 60, 75 (1989).

4. Savannah River Site Environmental Reports

\begin{tabular}{cc} 
Year & Report Number \\
\cline { 2 - 2 } 1985 & DPSPU 86-30-1 \\
1986 & DPSPU 87-30-1 \\
1987 & DPSPU 88-30-1 \\
1988 & WSRC-RP-89-59 \\
1989 & WSRC-IM-90-60 \\
1990 & WSRC-IM-91-28 \\
1991 & WSRC-TR-92-186 \\
1992 & WSRC-TR-93-075 \\
1993 & WSRC-TR-94-075 \\
1994 & WSRC-TR-95-075 \\
1995 & WSRC-TR-96-075
\end{tabular}

5. M. Stuiver and P.D. Quay, "Changes in Atmospheric Carbon-14 Attributed to a Variable Sun", Science 207, 11 (1980). 


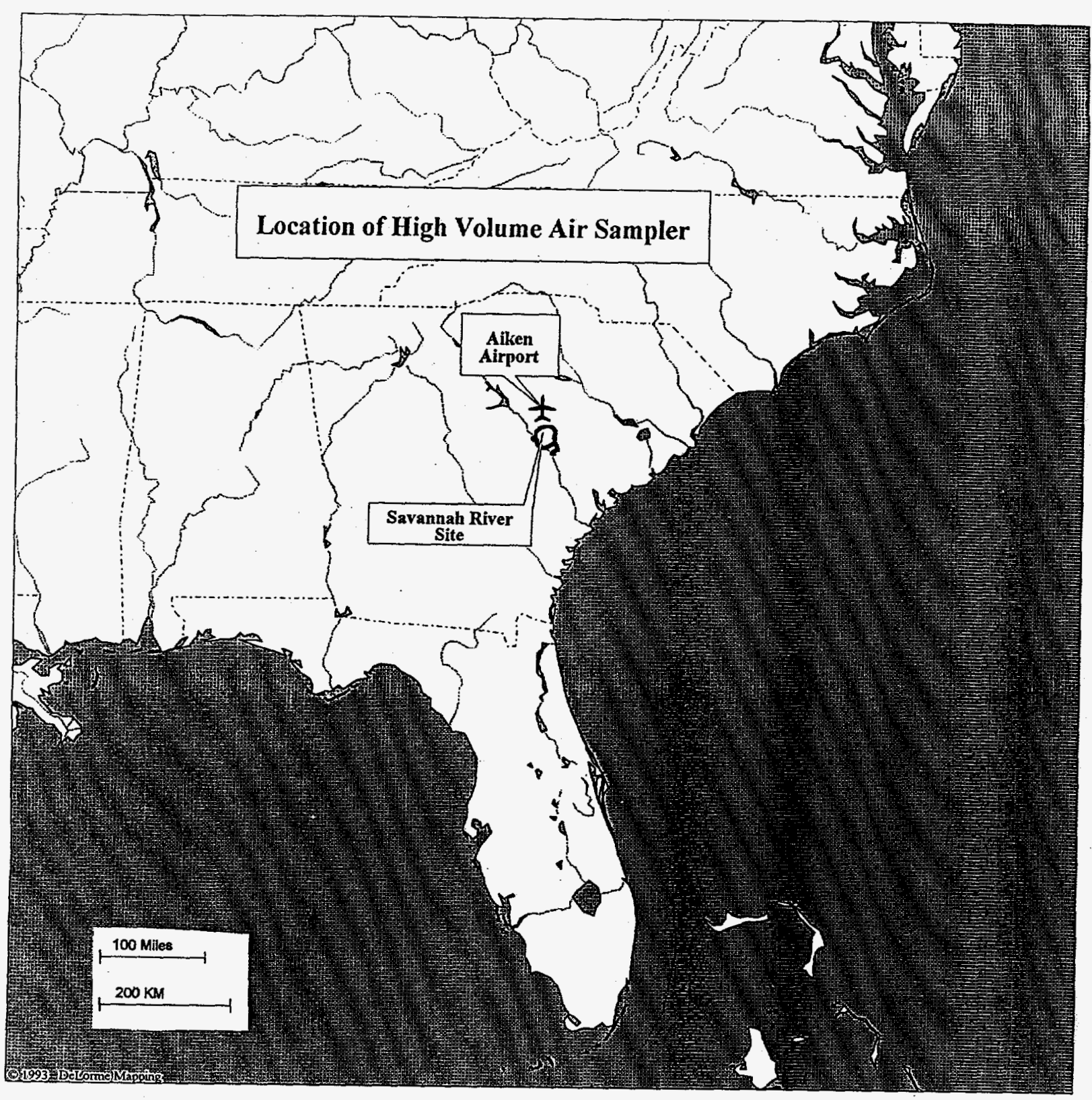

FIGURE 1. Location of Air Sampler 


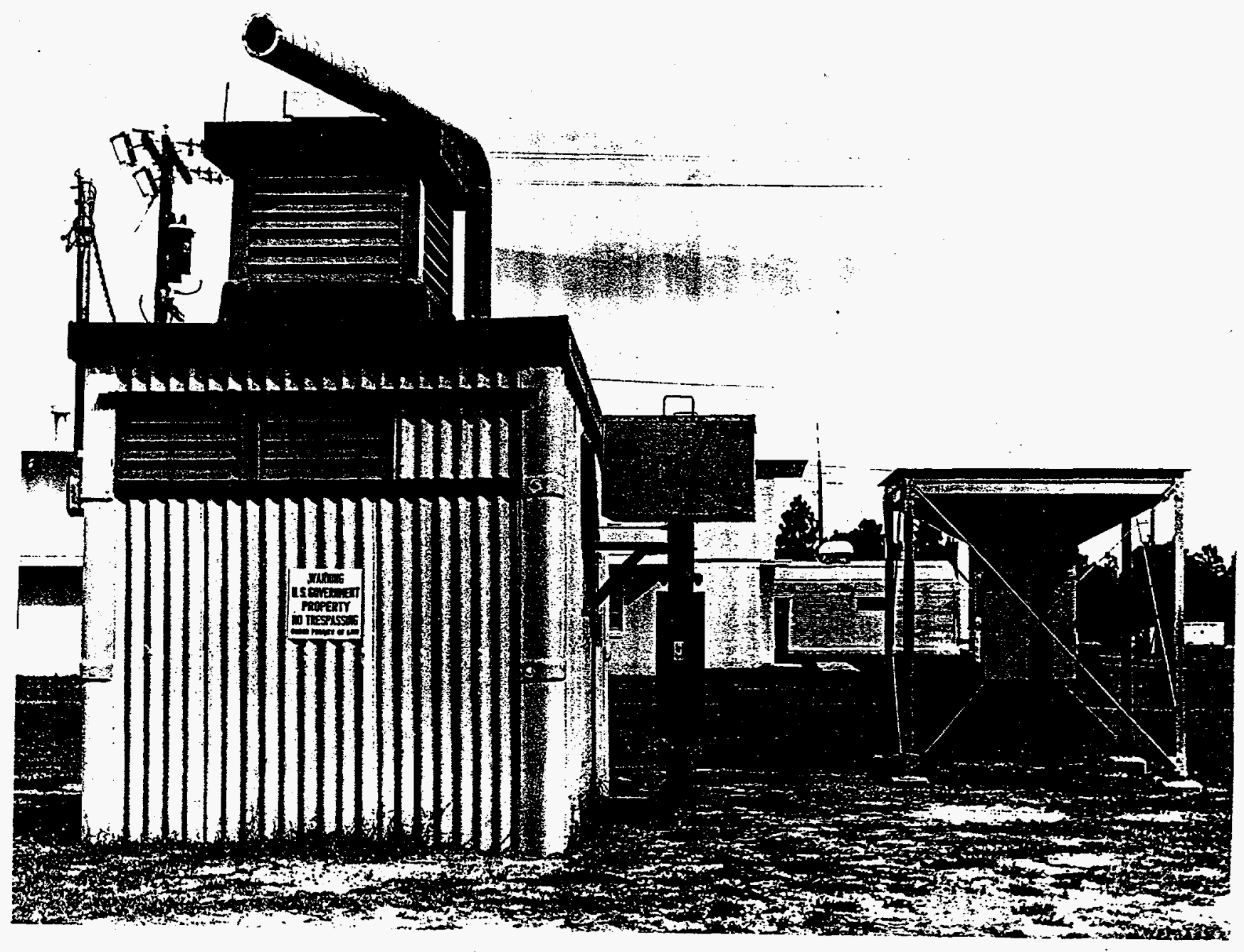

FIGURE 2. Air sampler system

The air sampler system consitiutes the corregated shed on left and associated equipment. Air passes through the

filter, which is located in the elevated box to the right of the shed; then the air passes through the pump within the shed and is exhausted from the pipe on top of the shed. 


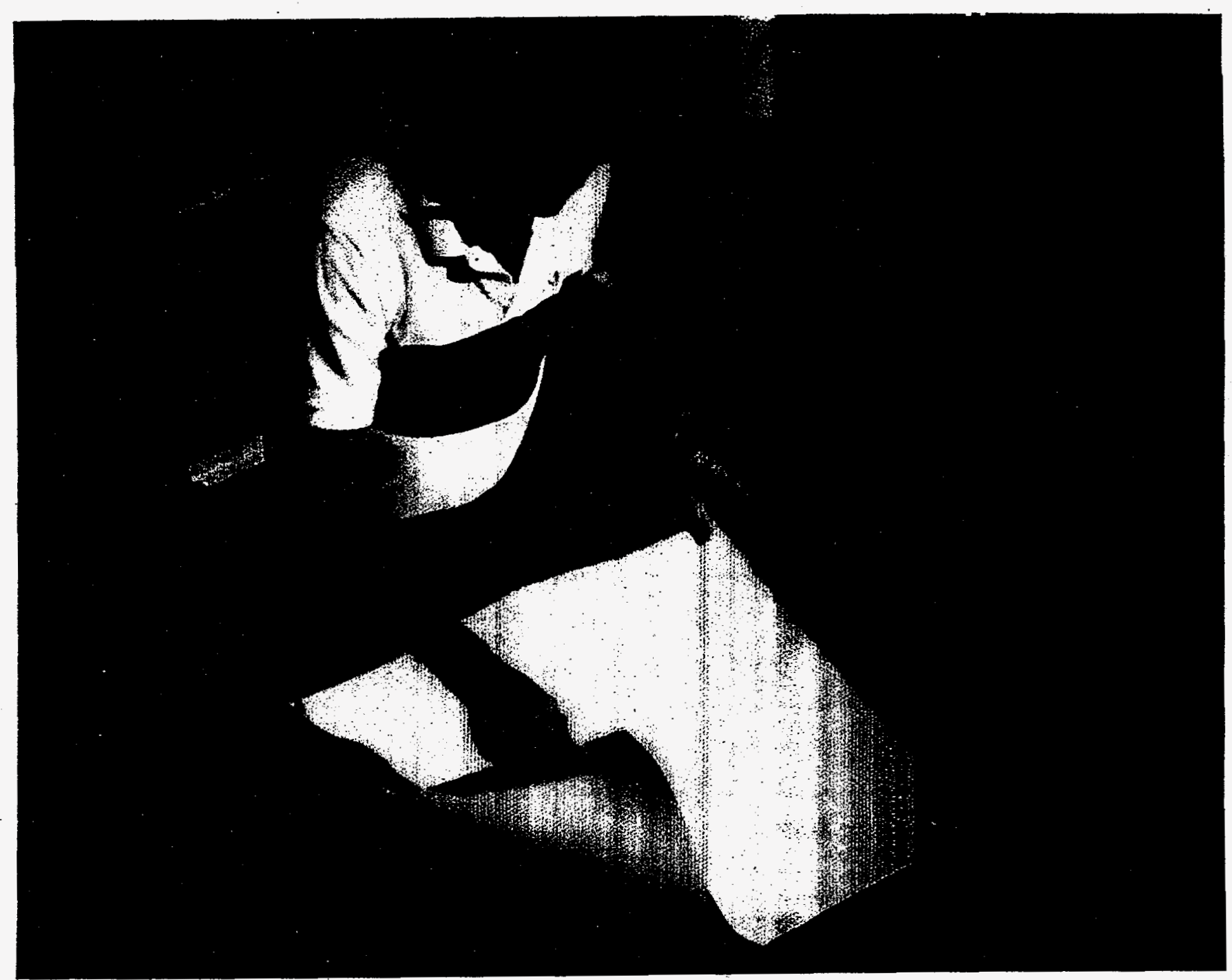

FIGURE 3. Cellulose Filter 


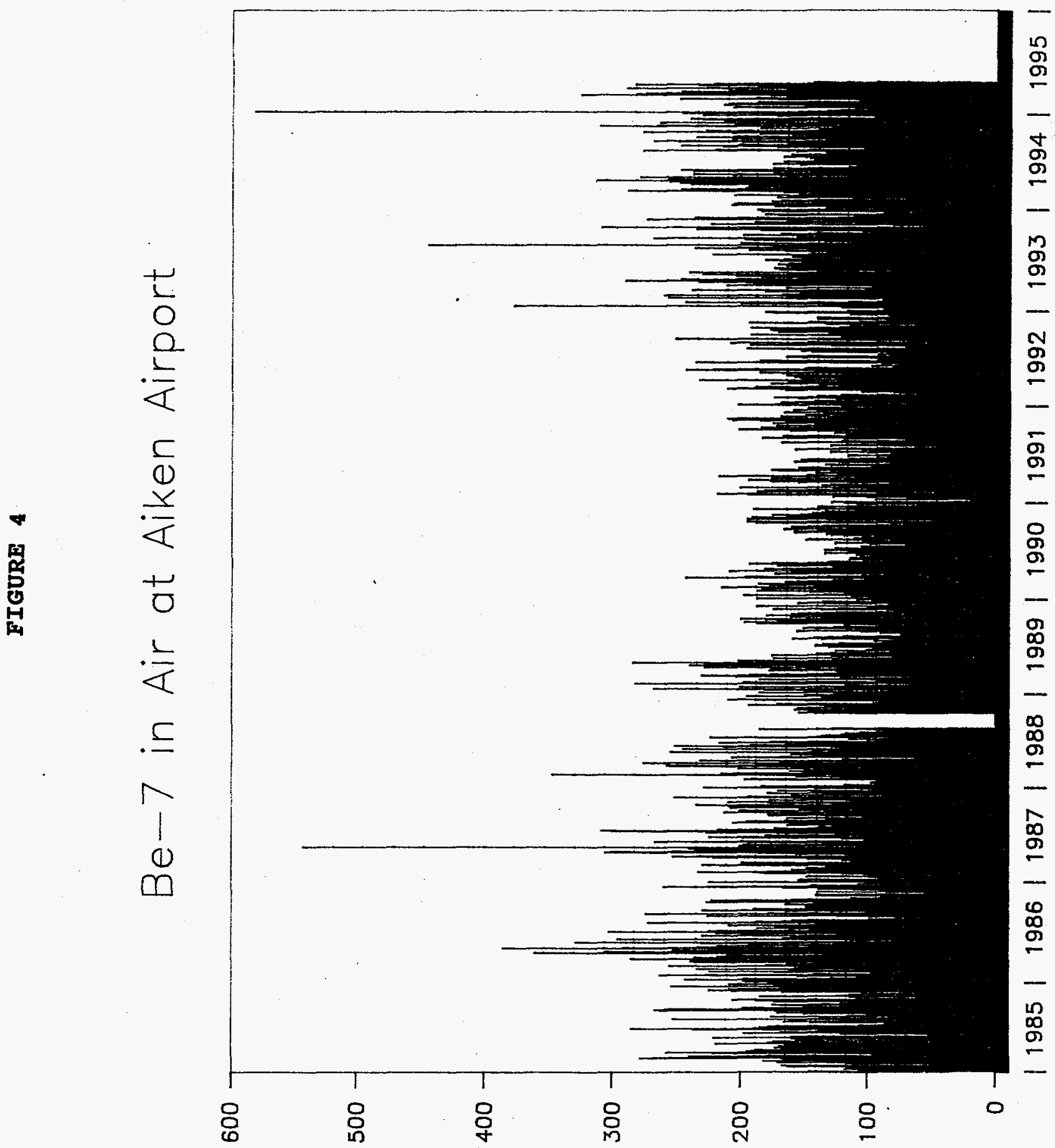

Eut/ot 


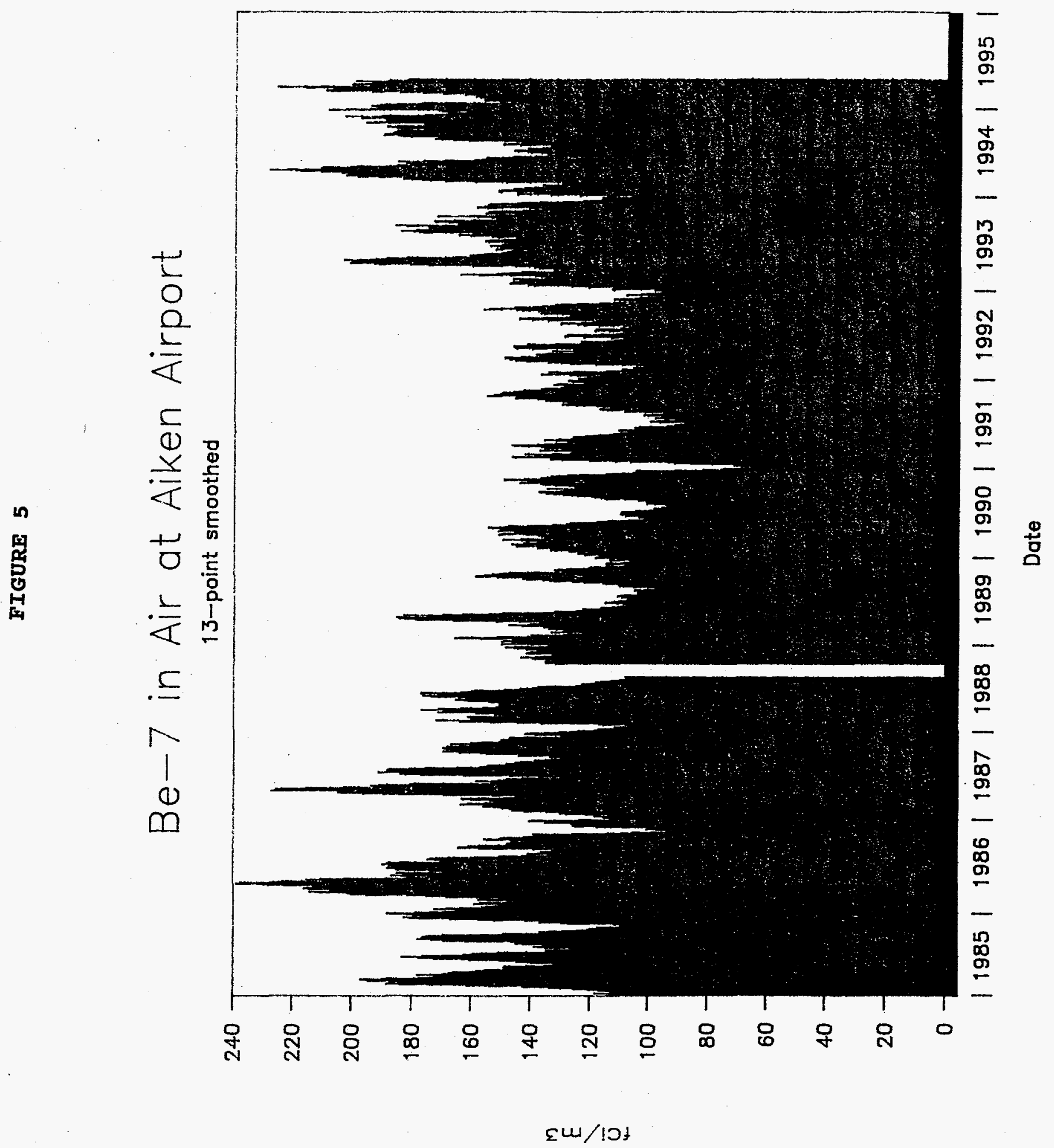


0

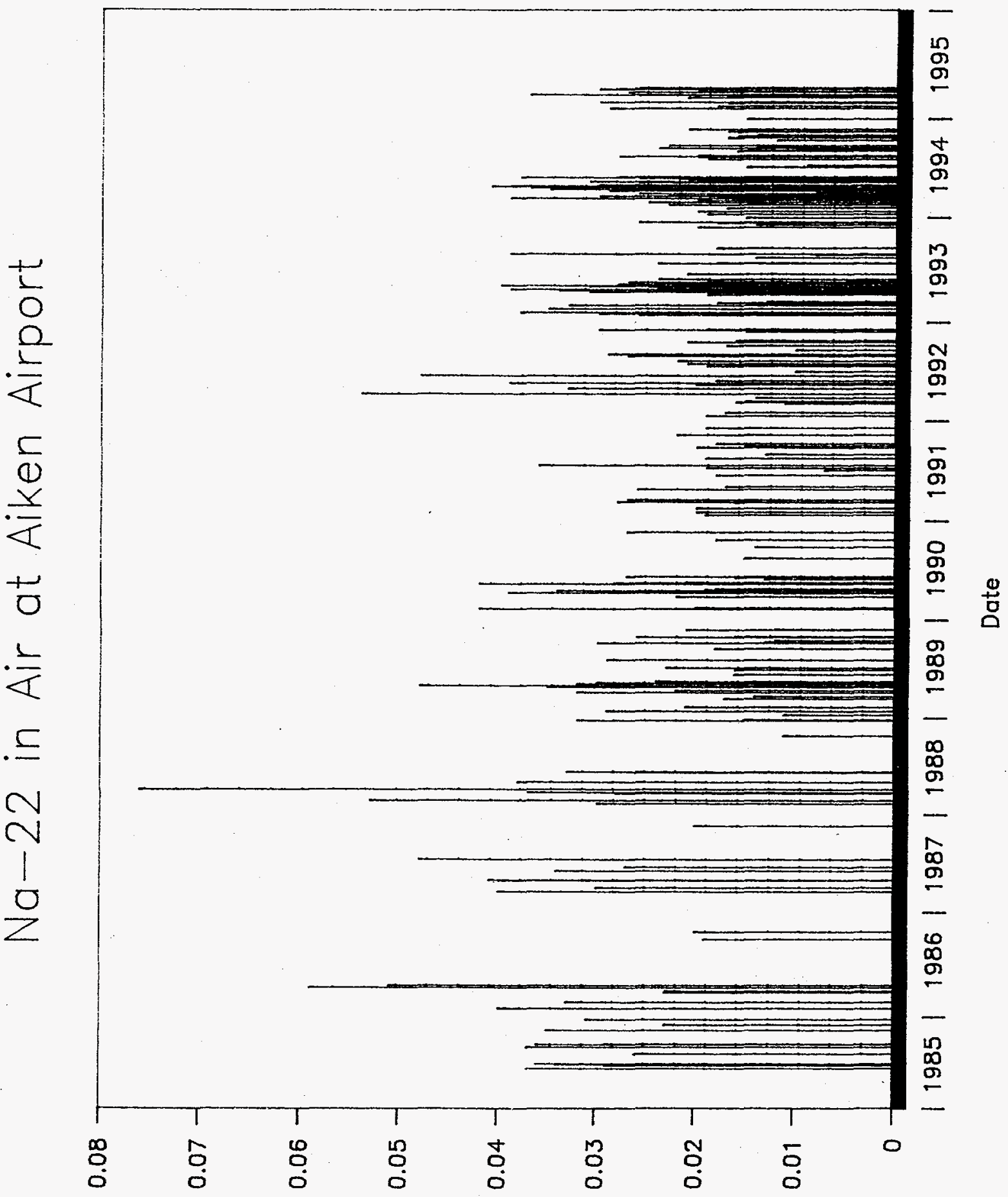

$\varepsilon u / ! O t$ 
FIGURE 7

\section{Co-60 in Air at Aiken Airport}

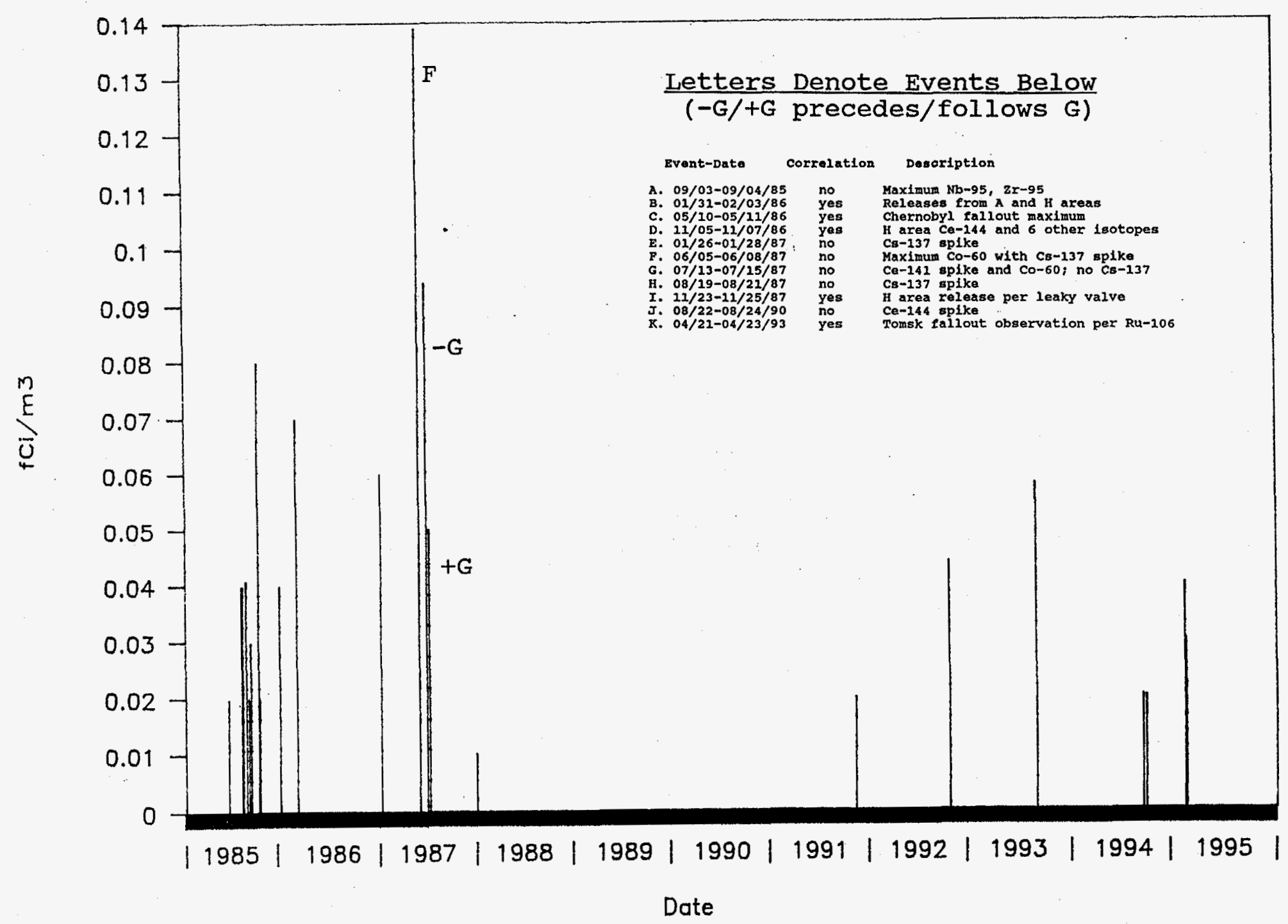


FIGURE 8

\section{Nb-95 in Air at Aiken Airport}

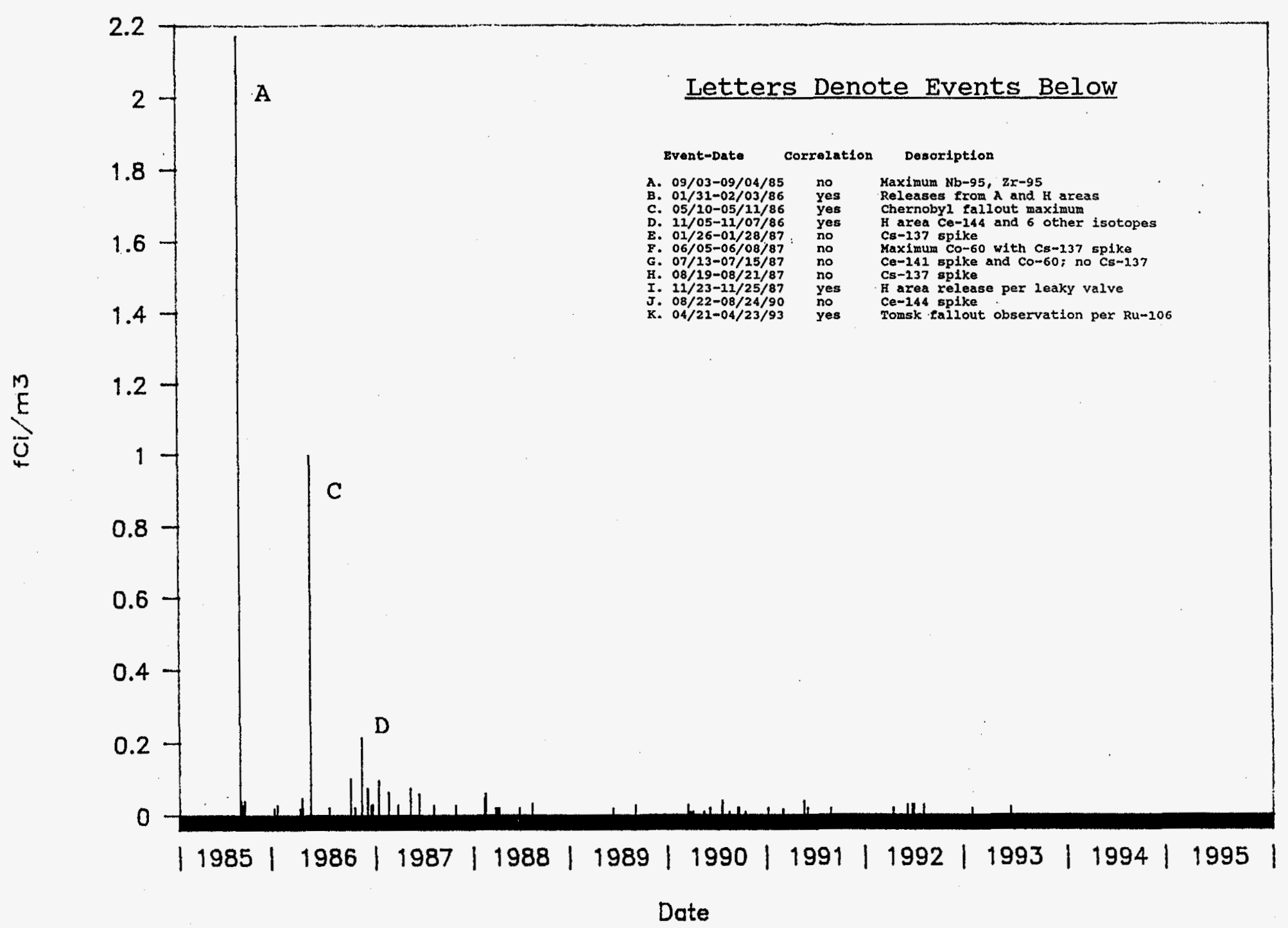


FIGURE 9

\section{Zr-95 in Air at Aiken Airport}

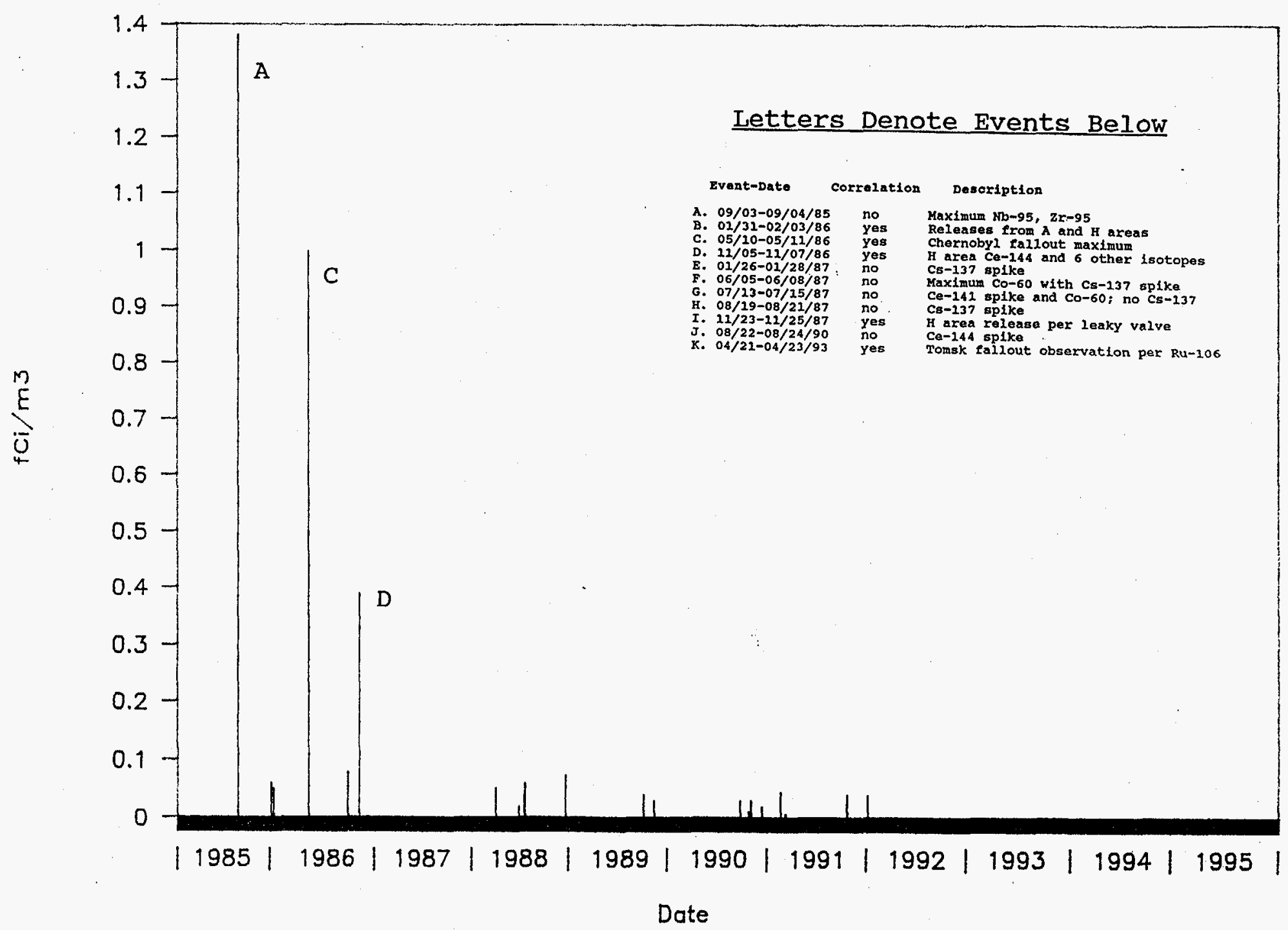


FIGURE 10

\section{Ru-103 in Air at Aiken Airport}

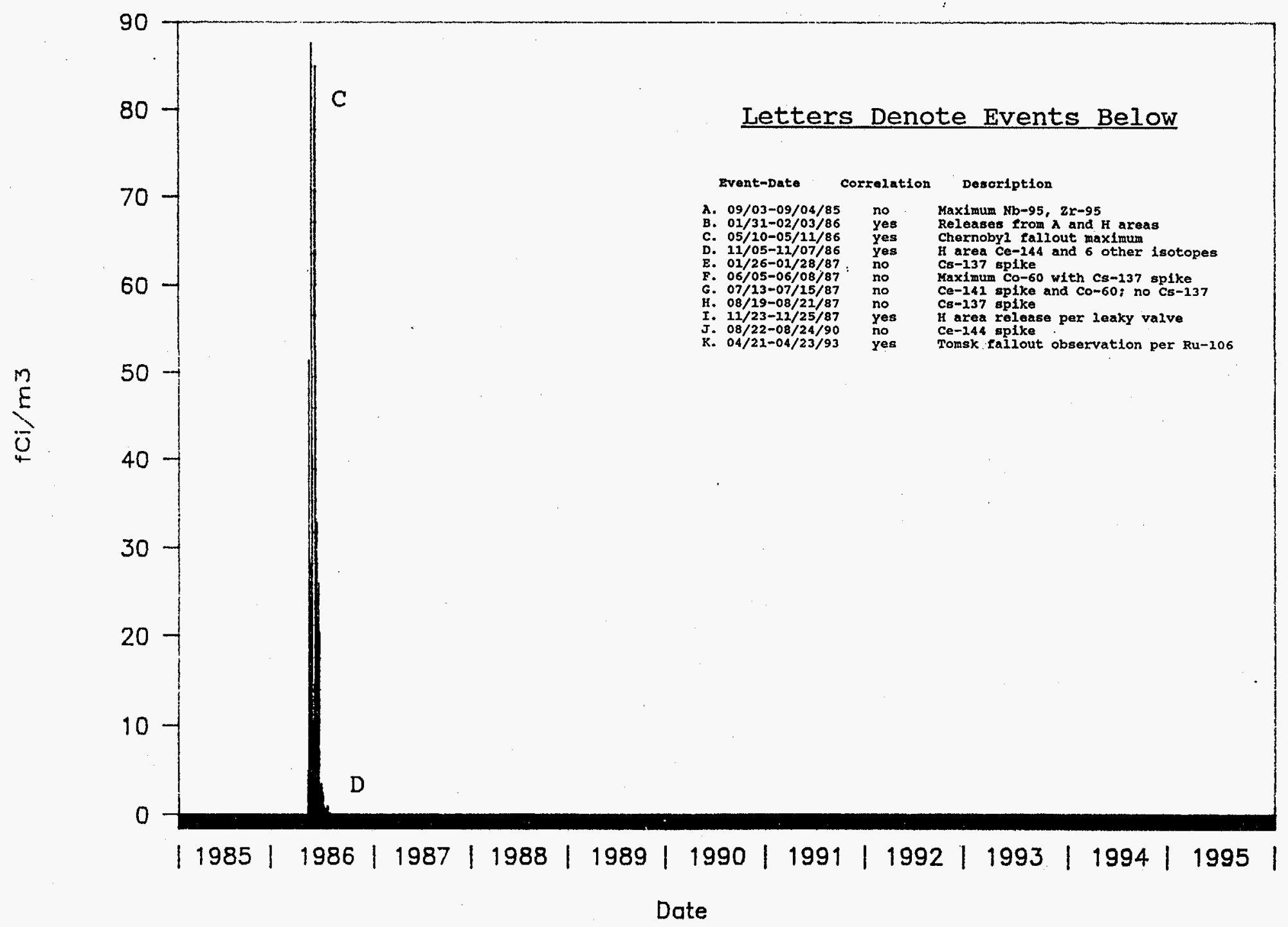


FIGURE 11

\section{Ru-106 in Air at Aiken Airport}

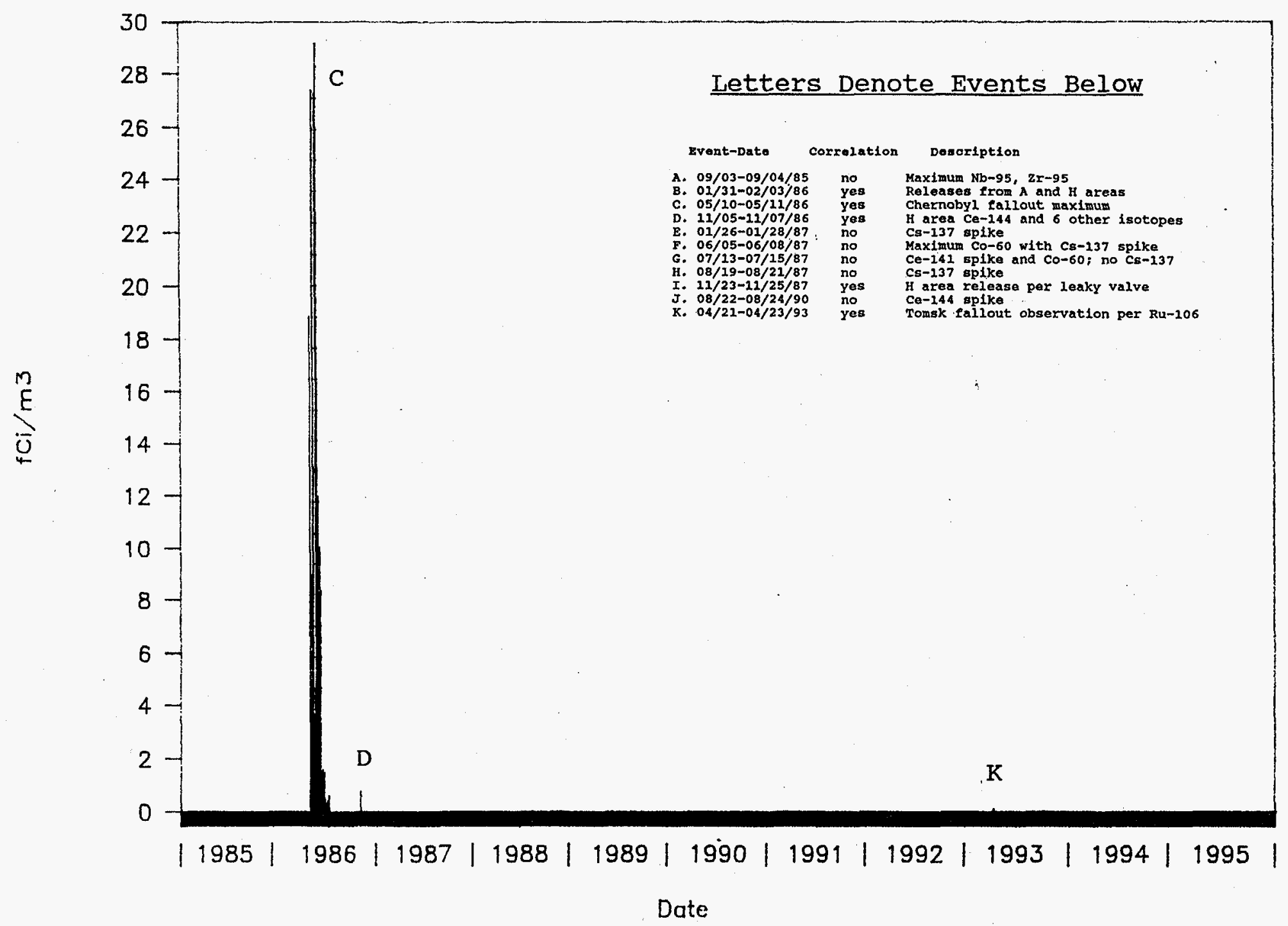


FIGURE 12

\section{Cs-134 in Air at Aiken Airport}

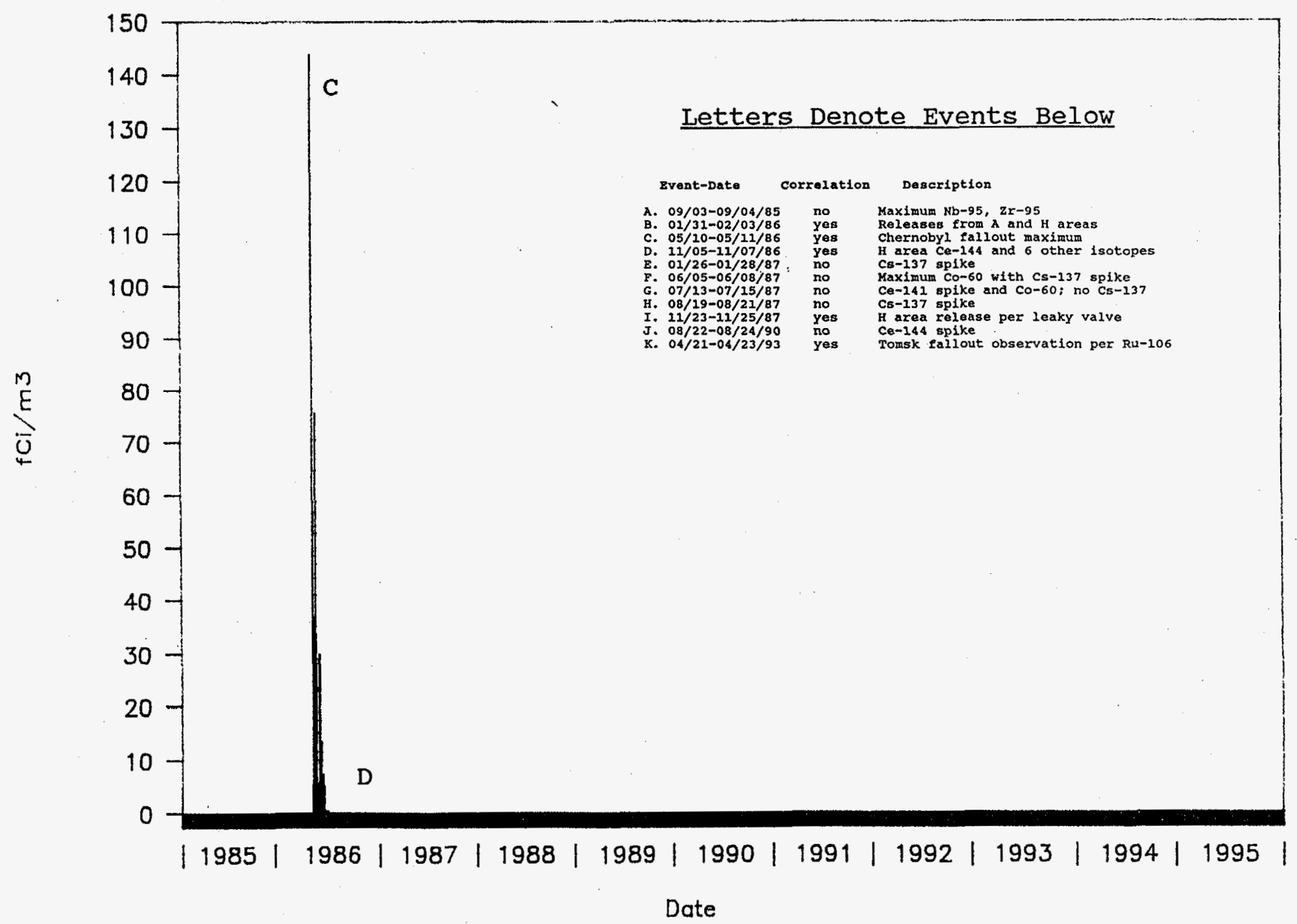


FIGURE 13

\section{Cs-137 in Air at Aiken Airport}

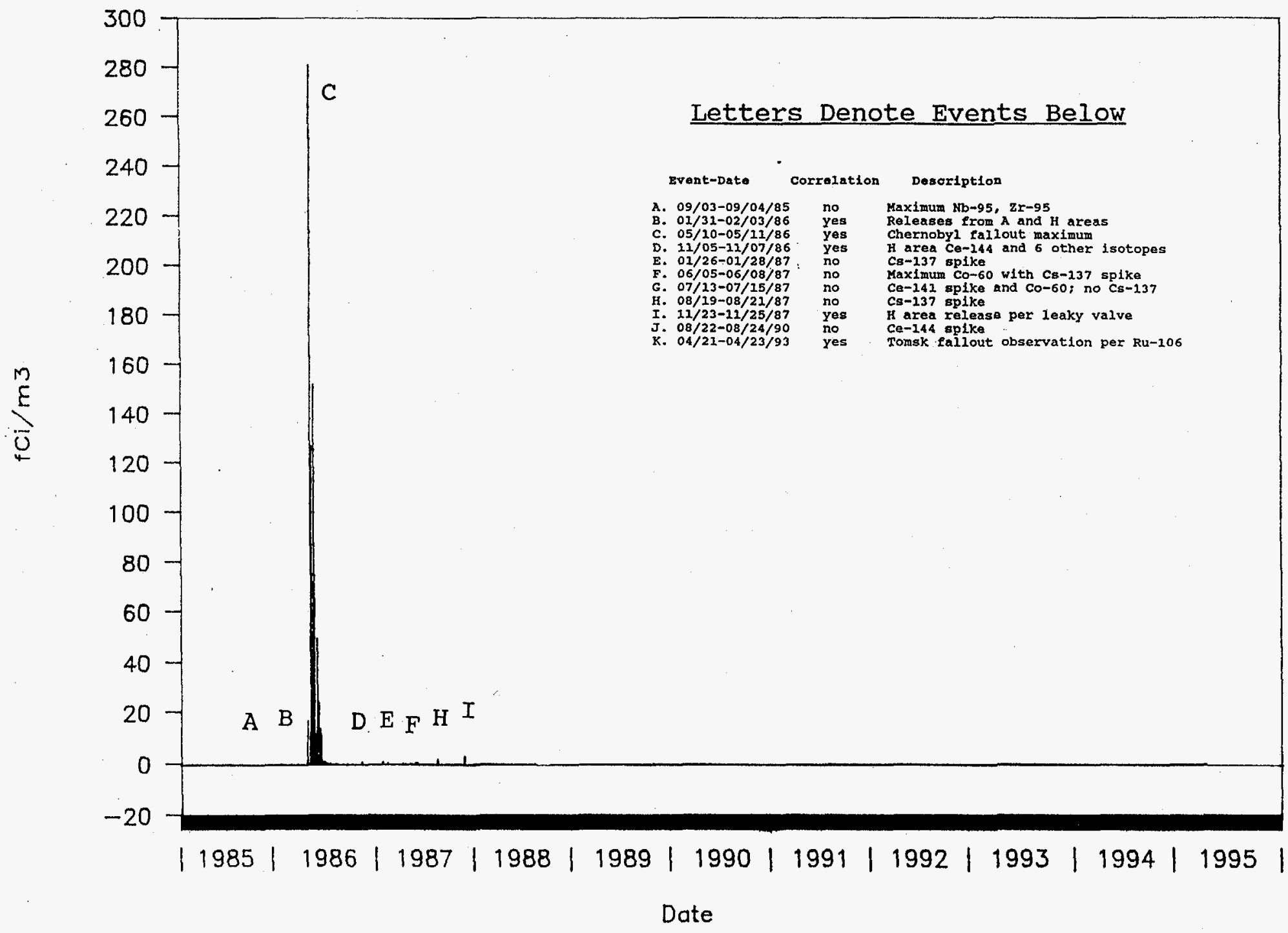




\section{Cs-137 in Air at Aiken Airport}

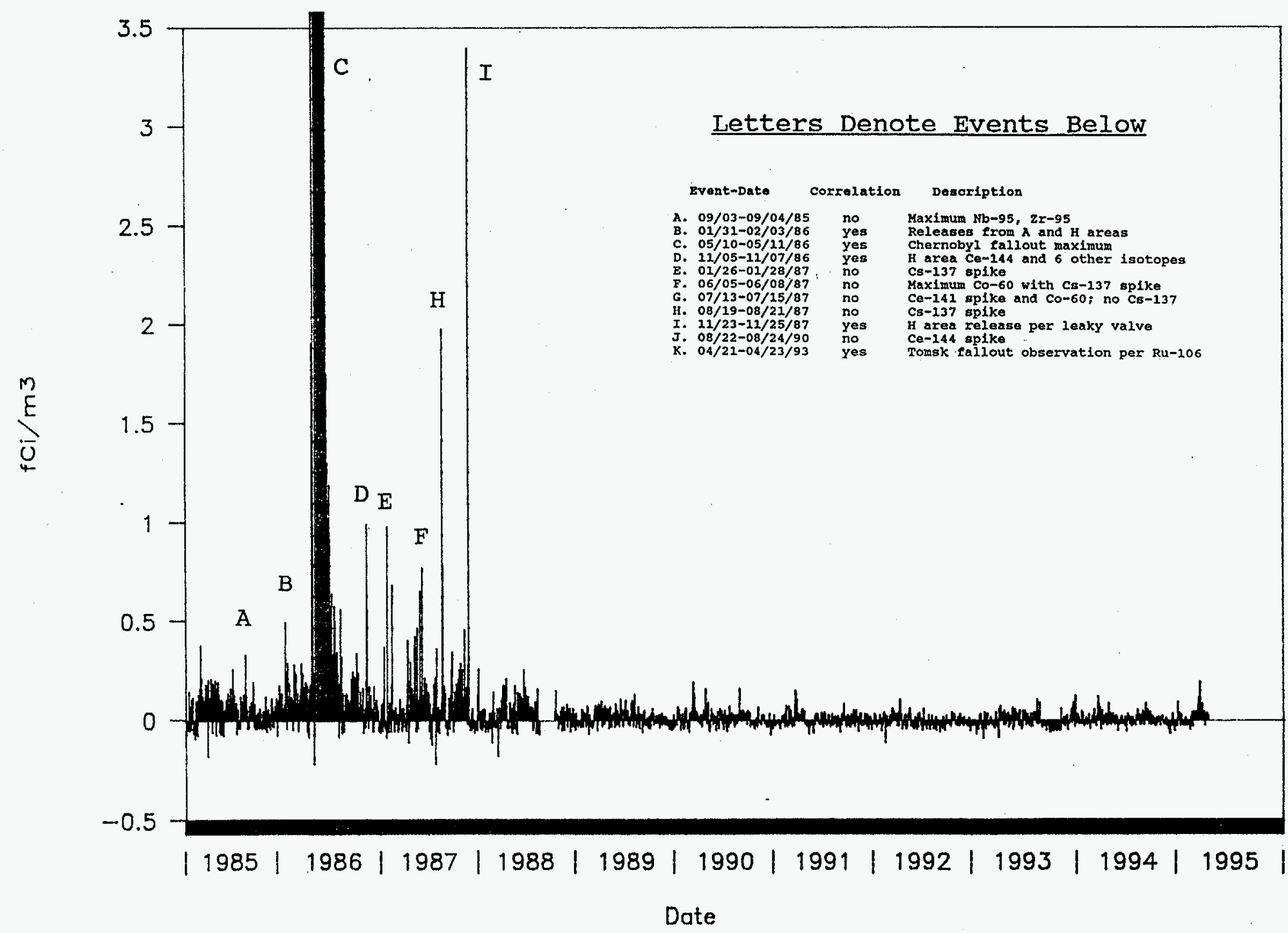


FIGURE 15

\section{Ce-141 in Air at Aiken Airport}

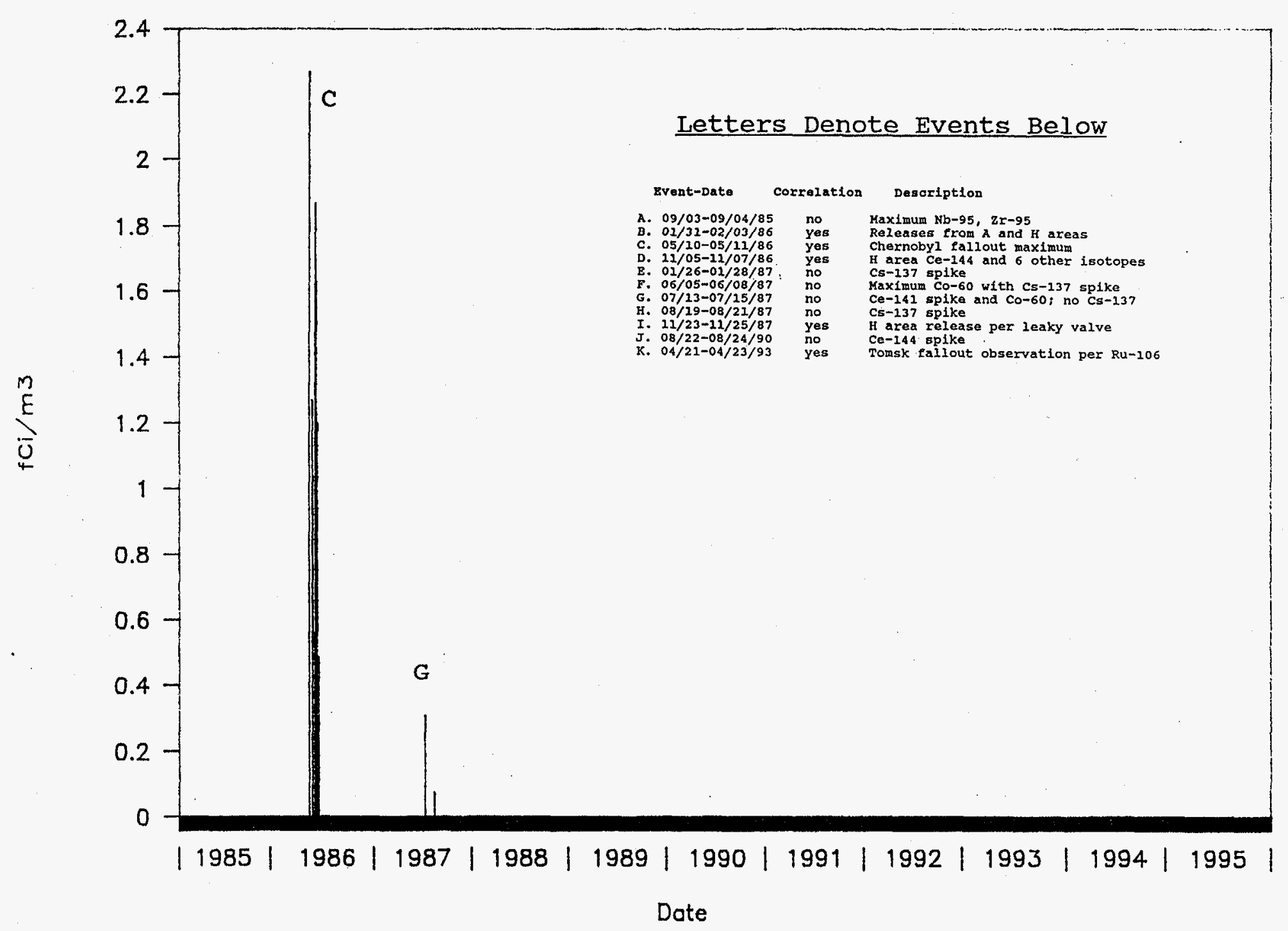




\section{Ce-144 in Air at Aiken Airport}

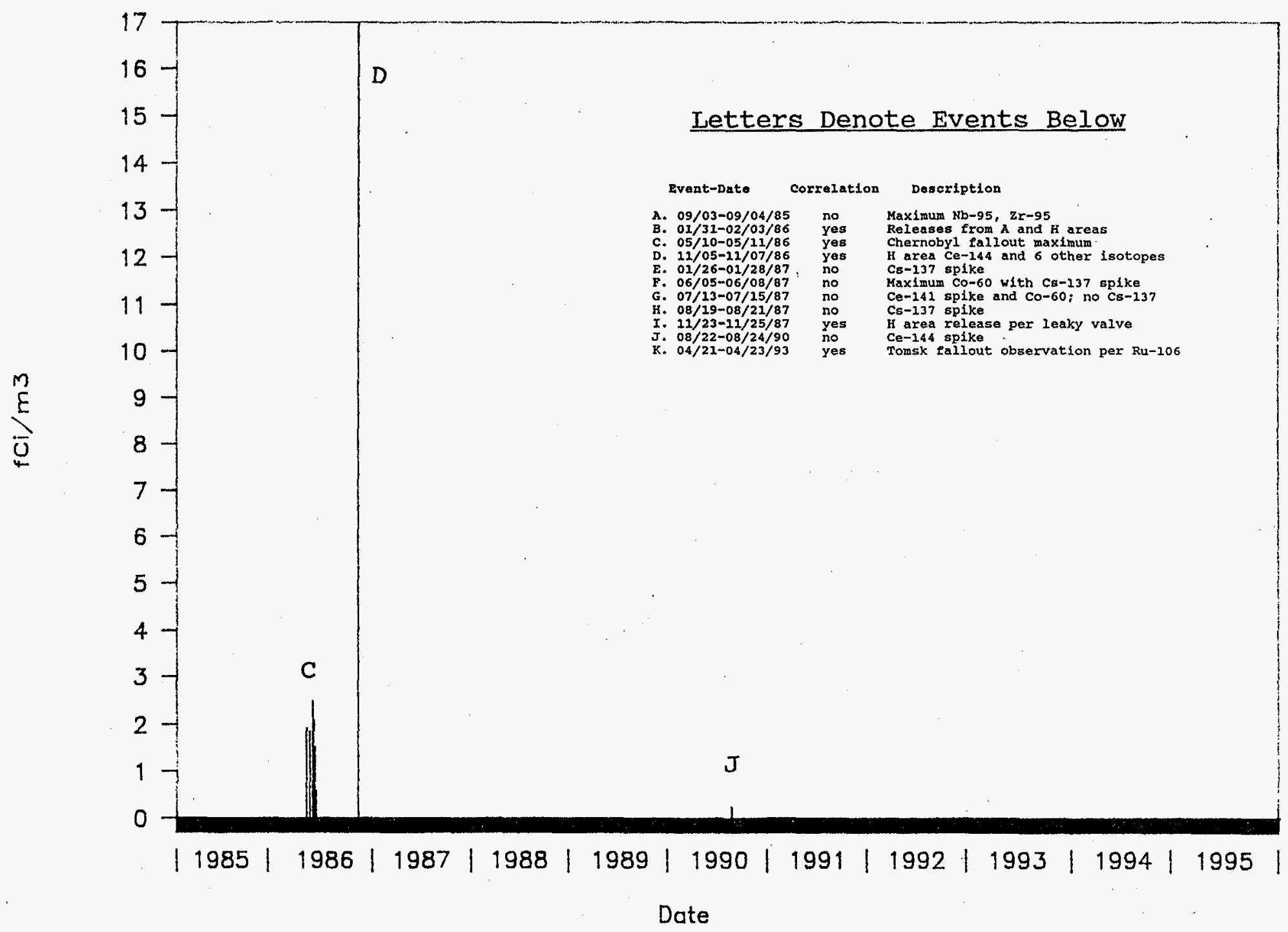




\section{Appendix A. Data Distribution Analysis}

The present work includes radioisotopic measurements that appear to be detected at random levels but only for a fraction of the observations. A normal probability distribution for such observations is shown below:

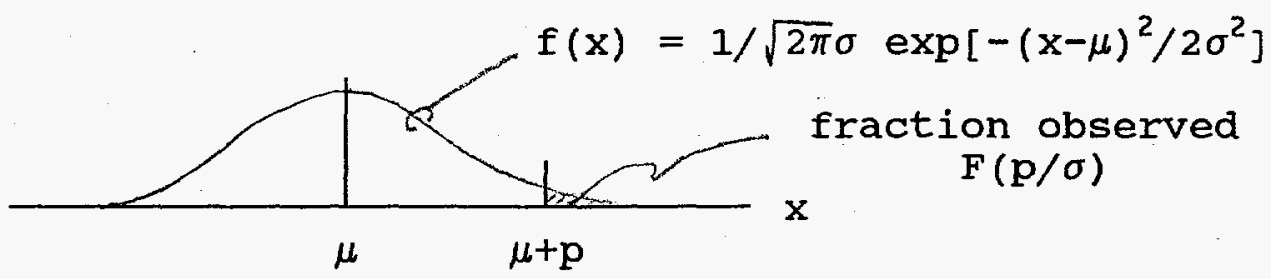

It is noted that the true mean $\mu$ of this distribution is below the detection threshold; it is obviously lower than the mean $U$ of the observed measurements, per

$$
\begin{aligned}
& \mathrm{U}={ }_{\mu f \mathrm{p}}^{\infty} \mathrm{xf}(\mathrm{x}) \mathrm{dx} / \mathrm{F}(\mathrm{p} / \sigma) \\
& \mathrm{U}=\mu+\sigma / \sqrt{2 \pi} \exp \left[-\mathrm{p}^{2} / 2 \sigma^{2}\right] / \mathrm{F}(\mathrm{p} / \sigma)
\end{aligned}
$$

If the sigma $S$ of the observed measurements is calculated, we may write

$$
\begin{aligned}
& \mathrm{U}=\mu+\mathrm{S}(\sigma / \mathrm{S}) 1 / \sqrt{2 \pi} \exp \left[-\mathrm{p}^{2} / 2 \sigma^{2}\right] / \mathrm{F}(\mathrm{p} / \sigma) \\
& \mathrm{U}=\mu+\mathrm{S} \mathrm{G}(\sigma / \mathrm{S}, \mathrm{p} / \sigma)
\end{aligned}
$$

where we note that we may calculate $G(\sigma / S, p / \sigma)$ simply from the knowledge of the fraction of fluctuations $F(p / \sigma)$ above the detection limit. A BASIC program for this is given with example calculations on pages $A-2$ and $A-3$.

As an example, we show the calculation for the $\mathrm{Na}-22$ data, assuming that it is log-normally distributed:

Data from observed detection:

$$
\begin{aligned}
\mathrm{F}(\mathrm{p} / \sigma) & =0.151 \text { or } 15.1 \% \\
\mathrm{U} & =-1.65=\log \left(.0224 \mathrm{fCi} / \mathrm{m}^{3}\right) \\
\mathrm{S} & =0.1785=\mathrm{std} \text { of } \log \left(\mathrm{x} \mathrm{fCi} / \mathrm{m}^{3}\right) \text { values }
\end{aligned}
$$

Calculation using $G$ vs $F$ Calculations (page $A-3$ )

$$
\mathrm{G}(\sigma / \mathrm{S}, \mathrm{p} / \sigma)=3.50
$$

Calculation of $\mu=\log (v)$ and $v$

$$
\begin{aligned}
& \mu=U-S G(\sigma / S, p / \sigma)=-1.65-0.1785 \times 3.50=-2.27 \\
& V=10^{-2.27}=0.0054 \mathrm{fCi} / \mathrm{m}^{3}
\end{aligned}
$$

$$
A-1
$$


5 REM gausslim.bas

10 CLS

100 REM - setup main loop

110 INPUT"First Limit PS (sigs)";PSF

120 INPUT"Last Limit PS (sigs)";PSL

130 INPUT"Step Limit PS (sigs)";PSS

135 PRINT:PRINT

140 PRINT" Iimit $F=\%$ Total

150

Pimit $F=\%$ Total

AvgL

SigL

G"

160 PRINT

PS $100 * \mathrm{DI}$

SD

AV/SD"

170 FOR PS $=$ PSF TO PSL STEP PSS

200 REM - calcs inner loop - probability

$240 \mathrm{DI}=0$

250 FOR $\mathrm{X}=$ PS+.01 TO PS+10 STEP .02

$260 \mathrm{DI}=\mathrm{DI}+\operatorname{EXP}\left(-.5 * \mathrm{X}^{\wedge} 2\right)$

265 REM PRINT X,DI

270 NEXT X

$280 \mathrm{DI}=.02 * \mathrm{SQR}(1 /(2 * 3.14159)) * \mathrm{DI}$

290 REM PRINT DI

300 REM - calcs inner loop - average and sigma

305 REM:IF PS $=0$ THEN 240

$310 \mathrm{NI}=\operatorname{SQR}(1 /(2 * 3.14159)) * \operatorname{EXP}\left(-.5 * \mathrm{PS}^{\wedge} 2\right)$

$320 \mathrm{AV}=\mathrm{NI} / \mathrm{DI}: \mathrm{REM}$ assumes sigma $=1$

330 REM $A V=0:$ REM test

$345 \mathrm{VR}=0$

350 FOR $\mathrm{X}=$ PS+.01 TO PS+10 STEP .02

$362 \mathrm{VR}=\mathrm{VR}+((\mathrm{X}-\mathrm{AV}) \wedge 2) * \operatorname{EXP}\left(-.5 * \mathrm{X}^{\wedge} 2\right)$

370 NEXT $X$

$385 \mathrm{VR}=.02 * \mathrm{SQR}(1 /(2 * 3.14159)) * \mathrm{VR} / \mathrm{DI}$

$386 \mathrm{SD}=\mathrm{SQR}(\mathrm{VR})$

400 PRINT USING"

, SD, AV/SD

500 NEXT PS

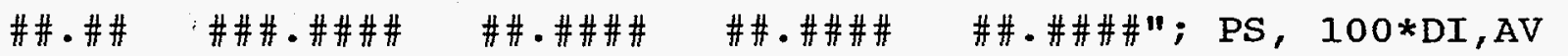


First Limit PS (sigs)? -5

Last Limit PS (sigs)? 5

Step Limit PS (sigs)? 1

$\begin{array}{crccc}\begin{array}{c}\text { Limit } \\ \text { PS }\end{array} & \begin{array}{c}\text { F=\%Total } \\ 100 * D I\end{array} & \begin{array}{c}\text { AVgL } \\ \text { AV }\end{array} & \begin{array}{c}\text { SigL } \\ \text { SD }\end{array} & \begin{array}{c}\text { G } \\ \text { AV } / S D\end{array} \\ -5.00 & 100.0000 & 0.0000 & 1.0000 & 0.0000 \\ -4.00 & 99.9969 & 0.0001 & 0.9997 & 0.0001 \\ -3.00 & 99.8651 & 0.0044 & 0.9933 & 0.0045 \\ -2.00 & 97.7252 & 0.0552 & 0.9415 & 0.0587 \\ -1.00 & 84.1349 & 0.2876 & 0.7935 & 0.3624 \\ 0.00 & 50.0000 & 0.7979 & 0.6028 & 1.3236 \\ 1.00 & 15.8651 & 1.5252 & 0.4462 & 3.4184 \\ 2.00 & 2.2748 & 2.3734 & 0.3380 & 7.0218 \\ 3.00 & 0.1350 & 3.2836 & 0.2656 & 12.3645 \\ 4.00 & 0.0032 & 4.2268 & 0.2160 & 19.5717 \\ 5.00 & 0.0000 & 5.1887 & 0.1807 & 28.7081\end{array}$

First Limit PS (sigs)? 1

Last Limit PS (sigs)? 1.1 step Limit PS (sigs)? .01

$\begin{array}{ccccc}\begin{array}{c}\text { Limit } \\ \text { PS }\end{array} & \begin{array}{c}\text { F=\%Total } \\ 100 * \mathrm{DI}\end{array} & \begin{array}{c}\text { AvgI } \\ \text { AV }\end{array} & \begin{array}{c}\text { SigL } \\ \text { SD }\end{array} & \begin{array}{c}\text { G } \\ \text { AV/SD }\end{array} \\ 1.00 & 15.8651 & 1.5252 & 0.4462 & 3.4184 \\ 1.01 & 15.6244 & 1.5332 & 0.4449 & 3.4464 \\ 1.02 & 15.3860 & 1.5412 & 0.4436 & 3.4746 \\ 1.03 & 15.1501 & 1.5493 & 0.4423 & 3.5030 \\ 1.04 & 14.9166 & 1.5573 & 0.4410 & 3.5315 \\ 1.05 & 14.6855 & 1.5654 & 0.4397 & 3.5602 \\ 1.06 & 14.4569 & 1.5734 & 0.4384 & 3.5891 \\ 1.07 & 14.2306 & 1.5815 & 0.4371 & 3.6180 \\ 1.08 & 14.0067 & 1.5896 & 0.4358 & 3.6472 \\ 1.09 & 13.7853 & 1.5977 & 0.4346 & 3.6765 \\ 1.10 & 13.5662 & 1.6058 & 0.4333 & 3.7059\end{array}$

ok 
The air filter data are tabulated in this appendix. The data associated with the events tabulated on page 3 are underlined and identified by event letters (A, B, ... K).

Except for Cs-137, only values that are above the detection limit are recorded. For Cs-137, "less-than" detection limits are denoted as negative values. Positive entries with only one significant digit are rough estimates for marginal detection. other positive entries have counting errors that are better than the determination ilmit of $\pm 33 \%$. Conservative counting error guidelines for detected values $V\left(\mathrm{fCi} / \mathrm{m}^{3}\right)$ for each isotope are given below.

\begin{tabular}{|c|c|c|c|}
\hline Isotope & Estim & at & $d \quad \sigma \quad\left(\mathrm{fci} / \mathrm{m}^{3}\right)$ \\
\hline $\mathrm{Be}-7$ & & $x$ & $(v / 100)^{1 / 2}$ \\
\hline $\mathrm{Na}-22$ & 0.004 & $\mathrm{x}$ & $(\mathrm{V} / 0.010)^{1 / 2}$ \\
\hline $\mathrm{Co}-6 \mathrm{O}$ & 0.004 & $\mathrm{x}$ & $(\mathrm{V} / 0.010)^{1 / 2}$ \\
\hline $\mathrm{Nb}-95 *$ & 0.005 & $\mathrm{x}$ & $(V / 0.010)^{1 / 2}$ \\
\hline $\operatorname{Zr}-95 *$ & 0.007 & $\mathrm{x}$ & $(\mathrm{V} / 0.010)^{1 / 2}$ \\
\hline $\mathrm{Ru}-103$ & 0.005 & $\mathrm{x}$ & $(\mathrm{V} / 0.010)^{1 / 2}$ \\
\hline $\mathrm{Ru}-106^{\circ}$ & 0.06 & $\mathrm{x}$ & $(v / 0.10)^{1 / 2}$ \\
\hline Cs -137 & 0.006 & $\mathrm{x}$ & $(\mathrm{V} / 0.010)^{1 / 2}$ \\
\hline $\mathrm{Ce}-141$ & 0.05 & $x$ & $(v / 0.10)^{1 / 2}$ \\
\hline $\mathrm{Ce}-144$ & 0.05 & $\mathrm{x}$ & $(v / 0.10)^{1 / 2}$ \\
\hline
\end{tabular}

* Estimated $\sigma$ can vary due to natural background effects 


$$
\begin{aligned}
& 861^{\circ} 0 \\
& \text { III.0 } \\
& 890^{\circ} 0 \\
& 000^{\circ} 0- \\
& 861 \% \\
& \text { औII0 } \\
& \text { हटा० } \\
& \text { SLIO } \\
& 681 \% \\
& 890^{\circ} 0- \\
& 980^{\circ} 0 \\
& 402^{\circ} 0 \\
& 660^{\circ} 0 \\
& 560 \% \\
& 011 \% \\
& 5020 \\
& 081^{\circ} \mathrm{O}- \\
& 891 \% \\
& 860^{\circ} 0 \\
& 381 \% \\
& \text { Q } 21 \% \\
& \angle 80^{\circ} 0 \\
& 960^{\circ} 0 \\
& 650^{\circ} 0 \\
& \text { LII'O } \\
& \text { OEI } 0 \\
& 0120 \\
& \text { I9E. } 0 \\
& 191^{\circ} 0
\end{aligned}
$$

\begin{tabular}{|c|c|}
\hline$t 6$ & $02 / 5-61 / 5$ \\
\hline 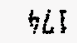 & $61 / 9-61 / 9$ \\
\hline EOI & $+1 / 5-01 / 5$ \\
\hline $8 L I$ & $01 / 5-8 / 5$ \\
\hline 022 & $8 / 5-9 / 5$ \\
\hline bit & $9 / \mathrm{s}-\mathrm{E} / \mathrm{S}$ \\
\hline 591 & $\varepsilon / 5-1 / 5$ \\
\hline $9 \mapsto$ & $58 / 1 / 5-b 2 / 4$ \\
\hline $0 L I$ & $6 z / t-9 z / t$ \\
\hline $6+1$ & $92 / h-6] / h$ \\
\hline 991 & $t 2 / t-2 己 / t$ \\
\hline$E L I$ & $22 / 4-66 / 4$ \\
\hline 891 & $6 / / t-L / / 7$ \\
\hline 60 t) & $C / / 4-g I / h$ \\
\hline B8I & $\xi I / h-z I / h$ \\
\hline 161 & $2 / / 4-01 / h$ \\
\hline 567 & $01 / t-8 / h$ \\
\hline 6? & $\theta / 6-8 / 6$ \\
\hline 892 & $\varepsilon / 4-1 / 4$ \\
\hline ટ己己 & $58 / 1 / h-b 2 / 8$ \\
\hline 502 & $6 己 / E-L 2 / E$ \\
\hline 96 & $4 己 / E-s] / E$ \\
\hline 8日 & $5 Z / E-Z Z / E$ \\
\hline $8 L I$ & $Z Z / E-1) Z / E$ \\
\hline In & $02 / E-8 I / E$ \\
\hline 812 & $\bar{B} / / E-G V / E$ \\
\hline 892 & $S T / E-E I / E$ \\
\hline BLE & $\varepsilon / / \varepsilon-11 / \varepsilon$ \\
\hline 691 & II/E-B/E \\
\hline 861 & $B / E-9 / E$ \\
\hline 991 & $9 / E-h / \varepsilon$ \\
\hline 691 & $\hbar / \varepsilon-1 / \varepsilon$ \\
\hline 281 & $58 / 1 / \varepsilon-L 2 / 2$ \\
\hline IEI & $L 己 / 2-52 / 2$ \\
\hline $0+1$ & 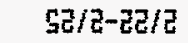 \\
\hline$U L I$ & $22 / 2-02 / 2$ \\
\hline 811 & $02 / 2-61 / 2$ \\
\hline हII & $6 / / 2-E V / 2$ \\
\hline is & $8 I / 2-I 1 / 2$ \\
\hline 891 & $11 / 2-8 / 2$ \\
\hline$\varepsilon E I$ & $8 / E-9 / 2$ \\
\hline 891 & $9 / 2-4 / 2$ \\
\hline $9 E$ & $\$ / 2-1 / 2$ \\
\hline LII & $58 / 1 / 2-0 E / 1$ \\
\hline 601 & DE/T-8ב/I \\
\hline 311 & $82 / 1-52 / 1$ \\
\hline 48 & $g z / I-\varepsilon z / I$ \\
\hline \multirow[t]{2}{*}{601} & E己/I- \\
\hline & $-81 / 1$ \\
\hline \multirow[t]{4}{*}{ 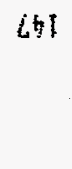 } & $91 / 1-0 \mathrm{H} / \mathrm{I}$ \\
\hline & $-9 / 1$ \\
\hline & $-8 / 1$ \\
\hline & $581-1 / 1$ \\
\hline
\end{tabular}

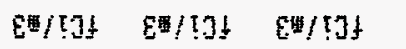

E11/II! $s h-q N$

EพII] 69-03 32-8n 


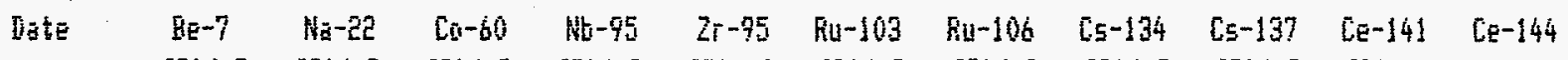

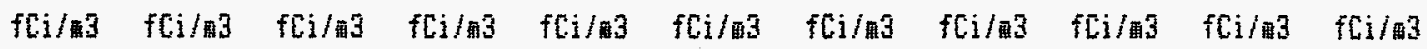

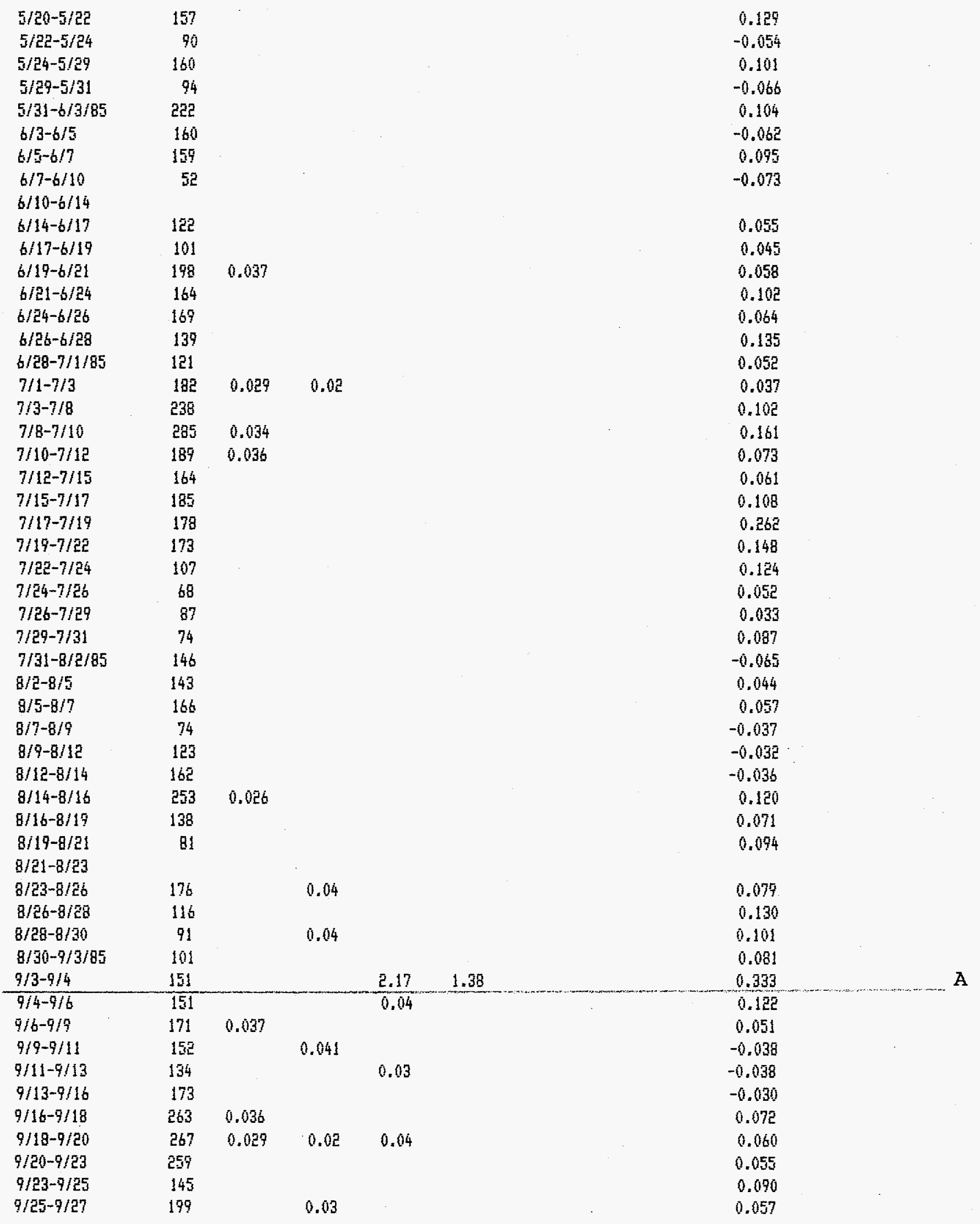




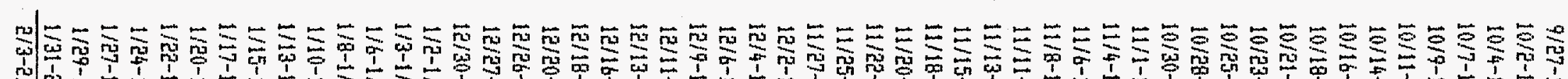

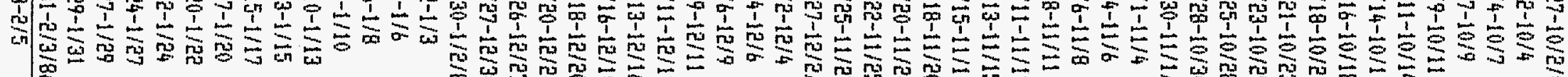

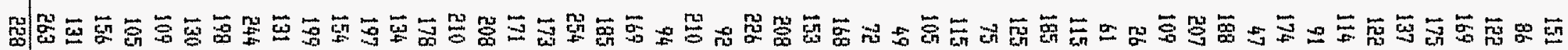

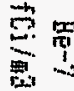




\begin{tabular}{|c|c|c|c|c|c|c|c|c|c|c|c|}
\hline Date & $\begin{array}{c}\mathrm{BE}-7 \\
\mathrm{fLi} \mathrm{i} / \mathbf{4} 3\end{array}$ & $\begin{array}{l}\mathrm{Na}-2 \overline{2} \\
\mathrm{i} \mathrm{C} / \mathrm{i} / \mathrm{m}\end{array}$ & $\begin{array}{l}c 0-60 \\
f C i / 43\end{array}$ & $\begin{array}{l}\mathrm{Mb}-95 \\
\mathrm{fCi} / \mathrm{m} 3\end{array}$ & $\begin{array}{l}2 \mathrm{r}-95 \\
\mathrm{fCi} / \mathrm{i} / 3\end{array}$ & $\begin{array}{l}\mathrm{Bu}-103 \\
\mathrm{fC \textrm {L } / \mathrm { A }} 3\end{array}$ & $\begin{array}{l}\mathrm{Ru}-10 \mathrm{6} \\
\mathrm{fCi} / \mathrm{it} 3\end{array}$ & $\begin{array}{l}{[\mathrm{s}-134} \\
\mathrm{f}[\mathrm{s} / \mathrm{m}]\end{array}$ & $\begin{array}{l}{[5-137} \\
f[1 / 1 / 3\end{array}$ & $\begin{array}{l}{[\mathrm{E}-141} \\
\mathrm{fCI} / \mathrm{A} / \mathrm{B}\end{array}$ & $\begin{array}{l}{[\mathrm{E}-144} \\
\mathrm{fCi} / \mathrm{A}^{-1} \mathrm{~B}\end{array}$ \\
\hline $2 / 5-2 / 7$ & 143 & & & & & & & & -0.039 & & \\
\hline $2 / 7-2 / 10$ & 97 & & & & & & & & 0.290 & & \\
\hline $2 / 10-2 / 12$ & 59 & & & & & & & & 0.043 & & \\
\hline $2 / 12-2 / 14$ & 174 & & & & & & & & 0.080 & & \\
\hline $2 / 14-2 / 18$ & 225 & & & & & & & & 0.187 & & \\
\hline $2 / 18-2 / 19$ & 162 & & & & & & & & 0.083 & & \\
\hline $2 / 19-2 / 21$ & 90 & & & & & & & & 0.116 & & \\
\hline $2 / 21-2 / 24$ & 107 & 0.033 & & & & & & & 0.113 & & \\
\hline $2 / 24-2 / 2 b$ & 235 & & & & & & & & 0.088 & & \\
\hline $2 / 26-2 / 29$ & 104 & & & & & & & & 0.100 & & \\
\hline $2 / 28-3 / 3 / 86$ & 158 & & & & & & & & -0.047 & & \\
\hline $3 / 3-3 / 5$ & 106 & & & & & & & & 0.082 & & \\
\hline $3 / 5-3 / 7$ & 154 & & & & & & & & 0.281 & & \\
\hline $3 / 7-3 / 10$ & $25 b$ & & & & & & & & 0.246 & & \\
\hline $3 / 10-3 / 12$ & 160 & & & & & & & & 0.233 & & \\
\hline $3 / 12-3 / 14$ & 101 & & & & & & & & 0.076 & & \\
\hline $3 / 14-3 / 17$ & 164 & & & & & & & & 0.037 & & \\
\hline $3 / 17-3 / 19$ & 141 & & & & & & & & 0.136 & & \\
\hline $3 / 19-3 / 21$ & 117 & & 0.07 & & & & & & -0.048 & & \\
\hline $3 / 21-3 / 24$ & 237 & & & & & & & & 0.082 & & \\
\hline $3 / 24-3 / 26$ & 240 & & & & & & & & 0.104 & & \\
\hline $3 / 2 b-3 / 27$ & 234 & & & & & & & & 0.125 & & \\
\hline $3 / 27-3 / 51$ & 193 & 0.023 & & & & & & & 0.160 & & \\
\hline $3 / 31-4 / 2 / 86$ & 240 & & & & & & & & 0.284 & & \\
\hline $4 / 2-4 / 4$ & 285 & .0 .023 & & & & & & & 0.237 & & \\
\hline $4 / 4-4 / 7$ & 219 & & & & & & & & 0.234 & & \\
\hline $4 / 7-4 / 9$ & 237 & & & & & & & & 0.167 & & \\
\hline $4 / 9-4 / 11$ & 215 & & & & & & & & 0.042 & & \\
\hline $4 / 11-4 / 14$ & 219 & & & 0.02 & & & & & 0.154 & & \\
\hline $4 / 14-4 / 18$ & 212 & & & & & & & & 0.190 & & \\
\hline $4 / 16-4 / 18$ & 91 & 0.059 & & & & & & & 0.126 & & \\
\hline $4 / 18-4 / 21$ & 156 & & & & & & & & 0.172 & & \\
\hline $4 / 21-4 / 23$ & 211 & & & 0.05 & & & & & -0.056 & & \\
\hline $4 / 23-4 / 25$ & 361 & 0.051 & & 0.02 & & & & & 0.103 & & \\
\hline $4 / 2 c_{-}-4 / 28$ & 143 & & & & & & & & -0.082 & & \\
\hline $4 / 2 g-4 / 30$ & 305 & & & & & & & & 0.161 & & \\
\hline $4 / 30-5 / 2 / 86$ & 227 & & & & & & & & 0.176 & & \\
\hline $5 / 2-5 / 3$ & 253 & & & & & & & & 0.145 & & \\
\hline $5 / 3-5 / 4$ & 255 & & & & & & & & 0.185 & & \\
\hline $5 / 4-5 / 5$ & 188 & & & & & & & & 1.910 & & \\
\hline $5 / 5-5 / 6$ & 386 & & & & & & & 0.243 & 17.000 & & \\
\hline $5 / 6-5 / 7$ & 291 & & & & & & & & 0.234 & & \\
\hline $5 / 7-5 / 8$ & 234 & & & & & & & & -0.220 & & \\
\hline $5 / 8-5 / 9$ & 146 & & & & & 0.54 & & & 0.201 & & \\
\hline $5 / 9-5 / 10$ & $11 !$ & & & & & 5.00 & & 5.6 & 11.500 & & \\
\hline $5 / 10-5 / 11$ & 219 & & & 1 & 1 & 51.4 & 18.9 & 144.0 & 281.000 & 2.27 & 1.94 \\
\hline $5 / 11-5 / 12$ & 193 & & & & & 28.6 & 10.1 & 57.0 & 127.000 & & \\
\hline $5 / 12-5 / 13$ & 127 & & & & & 10.7 & 3.9 & 8.8 & 17.800 & & \\
\hline $5 / 13-5 / 14$ & 120 & & & & & 13.8 & 3.4 & 16.6 & 37.400 & & \\
\hline $5 / 14-5 / 15$ & 232 & & & & & 28.1 & 9.0 & 37.0 & 72.300 & & \\
\hline $5 / 15-5 / 16$ & $32 \bar{y}$ & & & & & 97.6 & 27.4 & 75.8 & 152.000 & 1.27 & 1.86 \\
\hline $5 / 16-5 / 17$ & 164 & & & & & 40.4 & 13.1 & 34.0 & 65.300 & 1.04 & \\
\hline $5 / 17-5 / 18$ & 81 & & & & & 6.0 & 3.6 & 5.4 & 10.700 & & \\
\hline
\end{tabular}




\begin{tabular}{|c|c|c|c|c|c|c|c|c|c|c|c|}
\hline Date & $\begin{array}{c}8 \mathrm{E}-7 \\
\mathrm{f}[\mathrm{ij} / \mathrm{w} 3\end{array}$ & $\begin{array}{l}\mathrm{Na}-22 \\
\mathrm{HCl} / \mathrm{i}^{-3} 3\end{array}$ & $\begin{array}{r}60-60 \\
\mathrm{fCi} / \mathrm{m} 3\end{array}$ & $\begin{array}{r}\mathrm{Nb}-95 \\
\mathrm{fCi} / \mathrm{F}^{-3}\end{array}$ & $\begin{array}{l}2 \mathrm{r}-95 \\
\mathrm{iCi} / \mathrm{m}\end{array}$ & $\begin{array}{l}8 u-103 \\
\mathrm{fCi} / \mathrm{i} 3 \mathrm{~s}\end{array}$ & $\begin{array}{l}\text { Fu }-106 \\
\mathrm{fCi} / \mathrm{i} \text { 的 }\end{array}$ & $\begin{array}{l}{[E-134} \\
\mathrm{fLi} / \text { 的 }\end{array}$ & $\begin{array}{l}{[5-137} \\
f[\mathrm{j} / 1 \times 3]\end{array}$ & $\begin{array}{l}\text { Ce-141 } \\
\{[\mathrm{Ci} / \mathbf{T}\}\end{array}$ & $\begin{array}{l}\mathrm{Ce}-144 \\
\mathrm{fCi} / \mathbf{- 3} 3\end{array}$ \\
\hline $5 / 18-5 / 19$ & 114 & & & & & 10.6 & 3.6 & 4.7 & 11.600 & & \\
\hline $5 / 19-5 / 21$ & 150 & & & & & 14.7 & 4.7 & 5.0 & 11.100 & 0.56 & \\
\hline $5 / 21-5 / 23$ & 296 & & & & & 80.3 & 27.5 & 27.2 & 50.000 & 1.87 & 2.50 \\
\hline $5 / 23-5 / 25$ & 553 & & & & & 85.0 & 29.2 & $30 . \bar{z}$ & 54.800 & 1.63 & 2.10 \\
\hline $5 / 25-5 / 27$ & 154 & & & & & 29.1 & 10.1 & 10.5 & 22.900 & 0.91 & 1.25 \\
\hline $5 / 27-5 / 30$ & 167 & & & & & 32.9 & 12.0 & 13.7 & 24.200 & 1.20 & 1.53 \\
\hline $5 / 30-6 / 2 / 86$ & 161 & & & & & 8.2 & 3.6 & 2.3 & 4.800 & & \\
\hline $6 / 2-6 / 4$ & 158 & & & & & 22.1 & 8.3 & 5.7 & 12.300 & 0.29 & 0.58 \\
\hline $8 / 4-6 / 6$ & 149 & & & & & 26.0 & 10.1 & 7.7 & 14.200 & 0.49 & \\
\hline$b / 6-b / 9$ & 291 & & & & & 20.5 & 8.4 & 5.3 & 11.700 & & \\
\hline $6 / 9-6 / 11$ & 123 & & & & & 2.6 & 1.5 & 0.7 & 1.750 & & \\
\hline $6 / 11-6 / 13$ & 106 & & & & & 3.1 & & 0.5 & 1.300 & & \\
\hline $6 / 13-b / 16$ & 200 & & & & & 3.5 & 1.6 & 0.4 & 1.020 & & \\
\hline $6 / 16-6 / 18$ & 220 & & & & & 3.1 & 1.3 & 0.3 & 0.770 & & \\
\hline $6 / 19-6 / 23$ & 187 & & & & & $\tilde{E} .5$ & 1.2 & 0.5 & 1.190 & & \\
\hline $6 / 23-6 / 25$ & 303 & & & & & 2.1 & 1.5 & 0.4 & 0.920 & & \\
\hline $6 / 25-6 / 27$ & 235 & & & & & 0.9 & 0.5 & 0.17 & 0.480 & & \\
\hline $6 / 27-6 / 30$ & 219 & & & & & 0.7 & & 0.15 & 0.560 & & \\
\hline $6790-7 / 2 / 86$ & 145 & & & & & & & 0.26 & 0.640 & & \\
\hline $7 / \varepsilon-7 / 7$ & 133 & & & & & 0.319 & 0.328 & 0.07 & 0.213 & & \\
\hline $7 / 7-7 / 9$ & 162 & & & & & 0.634 & 0.331 & 0.13 & 0.319 & & \\
\hline $7 / 9-7 / 11$ & 187 & & & 0.02 & & 0.666 & 0.418 & 0.10 & 0.338 & & \\
\hline $7 / 11-7 / 14$ & 151 & & & & & 0.941 & 0.611 & 0.15 & 0.576 & & \\
\hline $7 / 14-7 / 16$ & 173 & & & & & 0.758 & & 0.22 & 0.502 & & \\
\hline $7 / 16-7 / 18$ & 164 & & & & & & & & 0.325 & & \\
\hline $7 / 15-7 / 21$ & 182 & & & & & 0.202 & & & 0.107 & & \\
\hline $7 / 21-7 / 23$ & 210 & & & & & 0.080 & & & 0.344 & & \\
\hline $7 / 23-7 / 25$ & 140 & & & & & 0.171 & & 0.10 & 0.237 & & \\
\hline $7 / 25-7 / 28$ & 124 & & & & & & & & 0.186 & & \\
\hline $7 / 28-7 / 30$ & 155 & & & & & & & & -0.081 & & \\
\hline $7 / 30-8 / 1 / 86$ & 272 & & & & & & & 0.03 & 0.179 & & \\
\hline $8 / 1-8 / 4$ & 187 & & & & & & & & 0.147 & & \\
\hline $8 / 4-8 / 6$ & 168 & & & & & & & 0.04 & 0.564 & & \\
\hline $8 / 6-8 / 8$ & 150 & & & & & & & & 0.320 & & \\
\hline $8 / 8-8 / 11$ & 160 & & & & & & & & -0.061 & & \\
\hline $8 / 11-6 / 13$ & 99 & & & & & & & & 0.140 & & \\
\hline $8 / 13-8 / 15$ & 56 & & & & & & & & 0.048 & & \\
\hline $8 / 15-8 / 18$ & 57 & & & & & & & & -0.052 & & \\
\hline $8 / 18-8 / 20$ & 79 & & & & & & & & 0.039 & & \\
\hline $8 / 20-8 / 22$ & 99 & & & & & & & 0.026 & 0.130 & & \\
\hline $8 / 2 R^{2}-8 / 25$ & 125 & & & & & & & 0.024 & 0.131 & & \\
\hline $8 / 25-8 / 27$ & हले? & & & & & & & & 0.132 & & \\
\hline $8 / 27-8 / 29$ & 111 & & & & & & & & 0.087 & & \\
\hline $8 / 29-9 / 2 / 86$ & 161 & & & & & & & 0.035 & 0.102 & & \\
\hline $9 / 2-9 / 3$ & 274 & & & & & & & & 0.060 & & \\
\hline $9 / 3-9 / 5$ & 178 & & & & & & & & 0.081 & & \\
\hline $9 / 5-9 / 8$ & 102 & & & & & & & 0.015 & 0.038 & & \\
\hline $9 / 8-9 / 10$ & 100 & & & & & & & & 0.098 & & \\
\hline $9 / 10-9 / 12$ & 130 & & & & & 0.025 & & 0.022 & 0.210 & & \\
\hline $9 / 12-9 / 15$ & 165 & & & & & & & & 0.117 & & \\
\hline $9 / 15-9 / 17$ & 231 & & & & & & & & 0.247 & & \\
\hline $9 / 17-9 / 19$ & 146 & & & & & & & & 0.134 & & \\
\hline $9 / 19-9 / 22$ & 190 & 0.017 & & & & & & & 0.111 & & \\
\hline
\end{tabular}




\begin{tabular}{|c|c|c|c|c|c|c|c|c|c|c|c|c|}
\hline Date & $\begin{array}{c}\text { EE-7 } \\
\mathrm{fEi} / \text { r }^{3} 3\end{array}$ & $\begin{array}{l}\mathrm{Ha}-\overline{z z} \\
\mathrm{fCi} / \text { - } 33\end{array}$ & $\begin{array}{l}\mathrm{Cu}-60 \\
\mathrm{fCi} / 3\end{array}$ & $\begin{array}{l}\text { Hit-95 } \\
\mathrm{fCi} / \mathbf{i} 3\end{array}$ & $\begin{array}{l}2 \mathrm{r}-4 \mathrm{~s} \\
\mathrm{fCH} / \mathbf{m}\end{array}$ & $\begin{array}{l}\text { Ru-103 } \\
\text { fCis/mi }\end{array}$ & $\begin{array}{l}\text { Ru-106 } \\
\text { fCi mas }\end{array}$ & $\begin{array}{l}\mathrm{C} 5-134 \\
\mathrm{fCi} / \overline{\mathrm{m}}\end{array}$ & $\begin{array}{l}\mathrm{C} 5-137 \\
\mathrm{iCi} / \mathrm{m}\end{array}$ & $\begin{array}{l}{[\mathrm{e}-14]} \\
\mathrm{f}[\mathrm{i} / \mathrm{m}]\end{array}$ & $\begin{array}{l}{[\mathrm{E}-144} \\
\mathrm{fCi} / \text { 政 } 3\end{array}$ & \\
\hline $9 / 22-9 / 24$ & 121 & & & & & & & 0.090 & 0.221 & & & \\
\hline $9 / 24-9 / 26$ & 150 & & & 0.102 & 0.079 & & & & 0.178 & & & \\
\hline $9 / 26-9 / 29$ & 147 & & & & & & & & 0.061 & & & \\
\hline $9 / 29-10 / 1 / 86$ & 113 & & & & & & & 0.018 & 0.343 & & & \\
\hline $10 / 1-10 / 3$ & 150 & & & & & & & & 0.103 & & & \\
\hline $10 / 3-10 / 6$ & 117 & & & & & & & & 0.241 & & & \\
\hline $10 / 6-10 / 8$ & 165 & & & & & & & & 0.059 & & & \\
\hline $10 / 8-10 / 10$ & 71 & & & & & & & & 0.048 & & & \\
\hline $10 / 10-10 / 13$ & 144 & & & 0.02 & & & & & 0.037 & & & \\
\hline $10 / 13-10 / 15$ & 103 & & & & & & & & 0.146 & & & \\
\hline $10 / 15-10 / 17$ & 228 & & & & & & & & 0.041 & & & \\
\hline $10 / 17-10 / 20$ & 204 & 0.020 & & & & & & & 0.053 & & & \\
\hline $10 / 20-10 / 22$ & 200 & & & & & & & & 0.055 & & & \\
\hline $10 / 22-10 / 24$ & 224 & & & & & & & & 0.163 & & & \\
\hline $10 / 24-10 / 2 ?$ & 160 & & & & & & & & -0.060 & & & \\
\hline $10 / 27-10 / 29$ & 74 & & & & & & & & -0.034 & & & \\
\hline $10 / 29-10 / 31$ & 114 & & & & & & & & 0.061 & & & \\
\hline $10 / 31-11 / 3 / 86$ & 51 & & & & & & & & -0.029 & & & \\
\hline $11 / 3-11 / 5$ & 90 & & & & & & & 0.026 & 0.201 & & & \\
\hline $11 / 5-11 / 7$ & 73 & & & 0.216 & 0.390 & 0.083 & 0.779 & 0.226 & 0.998 & & 17.0 & D \\
\hline $11 / 7-11 / 10$ & 141 & & & & & & & & 0.558 & & & \\
\hline $11 / 10-11 / 12$ & 142 & & & & & & & & 0.092 & & & \\
\hline $11 / 12-11 / 17$ & 110 & & & & & & & & -0.034 & & , & \\
\hline $11 / 17-11 / 19$ & 55 & & & & & & & & $0.17 \mathrm{E}$ & & & \\
\hline $11 / 19-11 / 21$ & 52 & & & & & & & & 0.061 & & & \\
\hline $11 / 21-11 / 24$ & 86 & & & & & & & & 0.084 & & & \\
\hline $11 / 24-11 / 26$ & 141 & & & & & & & & 0.127 & & & \\
\hline $11 / 26-12 / 1 / 86$ & 90 & & & & & & & & 0.057 & & & \\
\hline $12 / 1-12 / 3$ & 36 & & & 0.073 & & & & & 0.054 & & & \\
\hline $12 / 3-12 / 5$ & 112 & & & & & & & & -0.052 & & & \\
\hline $12 / 5-12 / 8$ & 140 & & & & & & & & -0.050 & & & \\
\hline $12 / 8-12 / 10$ & 133 & & & & & & & & 0.173 & & & \\
\hline $12 / 10-12 / 12$ & 37 & & & & & & & & 0.036 & & & \\
\hline $12 / 12-12 / 16$ & 167 & & & 0.03 & & & & & 0.039 & & & \\
\hline $12 / 16-12 / 17$ & 260 & & & & & & & & 0.503 & & & \\
\hline $12 / 17-12 / 19$ & 227 & & & 0.03 & & & & & 0.072 & & & \\
\hline $12 / 19-12 / 22$ & 145 & & & & & & & & 0.049 & & & \\
\hline $12 / 2 \overline{2}-12 / 2 b$ & 61 & & & & & & & & 0.051 & & & \\
\hline $12 / 26-12 / 29$ & 99 & & & & & & & & -0.045 & & & \\
\hline $12 / 29-12 / 31$ & 108 & & & & & & & & -0.053 & & & \\
\hline $12 / 31-1 / 2 / 87$ & 102 & & & & & & & & -0.059 & & & \\
\hline $1 / 2-1 / 5$ & 65 & & & & & & & & 0.035 & & & \\
\hline $1 / 5-1 / 7$ & 226 & & & & & & & & 0.081 & & & \\
\hline $1 / 7-1 / 9$ & 135 & & & & & & & & -0.050 & & & \\
\hline $1 / 9-1 / 12$ & 90 & & & 0.02 & & & & & 0.094 & & & \\
\hline $1 / 1 \overline{2}-1 / 14$ & 155 & & 0.06 & 0.095 & & & & & 0.101 & & & \\
\hline $1 / 14-1 / 16$ & 154 & & & & & & & & 0.372 & & & \\
\hline $1 / 16-1 / 19$ & $5 \hat{z}$ & & & & & & & & -0.038 & & & \\
\hline$|/ 17-1 / 2|$ & 92 & & & & & & & & -0.031 & & & \\
\hline $1 / 21-1 / 23$ & $7 !$ & & & & & & & & -0.050 & & & \\
\hline $1 / 23-1 / 26$ & 99 & & & & & & & & -0.084 & & & \\
\hline $1 / 2 b-1 / 2 \mathrm{a}$ & 149 & & & & & & & & 0.794 & & & $\mathbf{E}$ \\
\hline
\end{tabular}




\begin{tabular}{|c|c|c|c|c|c|c|c|c|c|c|c|}
\hline Date & $\begin{array}{c}\mathrm{BE}-7 \\
\mathrm{fCi} / \mathrm{i} 3\end{array}$ & $\begin{array}{c}\mathrm{Na}-22 \\
\mathrm{fCi} / \mathbf{1} 3\end{array}$ & $\begin{array}{l}{[0-60} \\
\mathrm{fCi} / \overline{-1} 3\end{array}$ & $\begin{array}{l}\mathrm{Hb}-95 \\
\mathrm{fCi} / \mathrm{mi}\end{array}$ & $\begin{array}{l}2 r-95 \\
f C i / 8-3\end{array}$ & $\begin{array}{l}\mathrm{Ru}-103 \\
\mathrm{fCi} / \mathbf{1 0 3}\end{array}$ & $\begin{array}{l}\text { Ru-106 } \\
\mathrm{fCi} / \text { 的 } 3\end{array}$ & $\begin{array}{l}\mathrm{Cs-134} \\
\mathrm{fCi} / \mathbf{- 1} 3\end{array}$ & $\begin{array}{l}{[5-137} \\
\mathrm{i}\left[\mathrm{i} /{ }_{-6} 3\right.\end{array}$ & $\begin{array}{l}\text { [e-141 } \\
\text { fCi/m }\end{array}$ & $\begin{array}{l}\text { CE-144 } \\
\mathrm{fCi} / \mathrm{m} 3\end{array}$ \\
\hline $1 / 30-2 / 2 / 97$ & 99 & & & & & & & & -0.057 & & \\
\hline $2 / 2-2 / 4$ & 103 & & & & & & & & 0.089 & & \\
\hline $2 / 4-2 / 6$ & 121 & & & & & & & & 0.039 & & \\
\hline $2 / 6-2 / 9$ & 181 & & & & & & & & 0.061 & & \\
\hline $2 / 9-2 / 11$ & 185 & & & & & & & & -0.053 & & \\
\hline $2 / 11-2 / 13$ & 234 & & & & & & & & 0.181 & & \\
\hline $2 / 13-2 / 17$ & 178 & & & & & & & & 0.687 & & \\
\hline $2 / 17-2 / 18$ & 54 & & & & & & & & 0.050 & & \\
\hline $2 / 18-2 / 2 \mathrm{c}$ & 146 & & & 0.063 & & & & & 0.044 & & \\
\hline ह/22-2/25 & 160 & & & & & & & & -0.053 & & \\
\hline อ/Е5-ट/:7 & 149 & & & & & & & & -0.053 & & \\
\hline $2 / 27-3 / 2 / 87$ & 124 & & & & & & & & 0.068 & & \\
\hline $3 / 2-3 / 4$ & 149 & & & & & & & & 0.041 & & \\
\hline $3 / 4-3 / 6$ & 133 & & & & & & & & -0.019 & & \\
\hline $3 / 6-3 / 9$ & 134 & & & & & & & & 0.030 & & \\
\hline $3 / 4-3 / 11$ & 123 & & & & & & & & -0.056 & & \\
\hline $3 / 11-3 / 13$ & 217 & & & & & & & & -0.042 & & \\
\hline $3 / 13-3 / 16$ & 231 & & & & & & & & 0.044 & & \\
\hline $3 / 16-3 / 1 \overline{4}$ & 187 & & & & & & & & 0.104 & & \\
\hline $3 / 18-3 / 20$ & 179 & & & & & & & & 0.054 & & \\
\hline $3 / 20-3 / 23$ & 200 & 0.040 & & & & & & & 0.058 & & \\
\hline $3 / 23-3 / 25$ & 114 & & & & & & & & -0.051 & & \\
\hline $3 / 25-3 / 27$ & 85 & & & 0.03 & & & & & -0.029 & & \\
\hline $3 / 27-3 / 30$ & 109 & & & & & & & & 0.082 & & \\
\hline $3 / 30-4 / 1 / 87$ & 121 & & & & & & & & 0.036 & & \\
\hline \multicolumn{12}{|l|}{$4 / 1-4 / 3$} \\
\hline $4 / 3-4 / 6$ & 148 & 0.030 & & & & & & & -0.020 & & \\
\hline $4 / 6-4 / 8$ & 111 & & & & & & & & $-0.05 !$ & & \\
\hline $4 / 8-4 / 10$ & हृ? & & & & & & & & 0.090 & & \\
\hline $4 / 10-4 / 13$ & 25.3 & & & & & & & & 0.150 & & \\
\hline $4 / 13-4 / 15$ & 234 & & & & & & & & 0.408 & & \\
\hline $4 / 15-4 / 16$ & 115 & & & & & & & & -0.110 & & \\
\hline $4 / 16-4 / 20$ & 33 & & & & & & & & -0.027 & & \\
\hline $4 / 20-4 / 22$ & 157 & & & & & & & & 0.200 & & \\
\hline $4 / 22-4 / 24$ & 149 & & & & & & & & 0.296 & & \\
\hline $4 / 24-4 / 27$ & 186 & & & & & & & & 0.067 & & \\
\hline $4 / 27-4 / 2 ?$ & 306 & & & & & & & & 0.163 & & \\
\hline $4 / 29-5 / 1 / 87$ & 301 & 0.040 & & & & & & & 0.071 & & \\
\hline $5 / 1-5 / 4$ & 253 & 0.041 & & & & & & & 0.128 & & \\
\hline $5 / 4-5 / 6$ & 237 & & & & & & & & 0.124 & & \\
\hline $5 / 6-5 / \overline{8}$ & 205 & & & & & & & & 0.386 & & \\
\hline $5 / 8-5 / 11$ & 236 & & & 0.077 & & & & & 0.426 & & \\
\hline $5 / 11-5 / 13$ & 241 & & & & & & & & 0.093 & & \\
\hline $5 / 13-5 / 15$ & 108 & & & & & & & & -0.052 & & \\
\hline $5 / 15-5 / 18$ & 543 & & & & & & & & 0.468 & & \\
\hline \multicolumn{12}{|l|}{$5 / 18-5 / 20$} \\
\hline $5 / 20-5 / 22$ & 14 & & & & & & & & -0.035 & & \\
\hline $5 / 22-5 / 26$ & 104 & & & & & & & & 0.105 & & \\
\hline $5 / 26-5 / 27$ & 202 & & & & & & & & 0.094 & & \\
\hline $5 / 27-5 / 29$ & 160 & & & & & & & & 0.120 & & \\
\hline $5 / 29-8 / 1 / 87$ & 199 & & & & & & & & 0.652 & & \\
\hline $6 / 1-6 / 3$ & 136 & & & & & & & & 0.130 & & \\
\hline $6 / 3-6 / 5$ & 117 & & & & & & & & 0.105 & & \\
\hline
\end{tabular}




\begin{tabular}{|c|c|c|c|c|c|c|c|c|c|c|c|}
\hline Date & $\begin{array}{c}\mathrm{Be}-7 \\
\mathrm{fCi} / \mathbf{m}^{3}\end{array}$ & $\begin{array}{l}\mathrm{Na}-22 \\
\mathrm{fCi} / \mathrm{m} 3\end{array}$ & $\begin{array}{r}c 0-60 \\
i[\mathrm{i} / \mathrm{i} / \mathrm{i}\end{array}$ & $\begin{array}{l}\mathrm{Nb}-9 \mathrm{~s} \\
\mathrm{f}[\mathrm{i} / \mathrm{g} / \mathrm{3}\end{array}$ & $\begin{array}{r}2 r-95 \\
f \mathrm{fi} / \mathbf{w}^{-3}\end{array}$ & $\begin{array}{l}\text { fu }-103 \\
\mathrm{fci} / \mathrm{i} 3\end{array}$ & $\begin{array}{l}\mathrm{Fu}-106 \\
\mathrm{fCi} / \overline{3}\end{array}$ & $\begin{array}{l}{[5-134} \\
f\left[1 /{ }_{1}\right]\end{array}$ & $\begin{array}{l}{[5-137} \\
f[\mathrm{~L} / \mathrm{m}]\end{array}$ & $\begin{array}{l}{[E-141} \\
{[C \mathrm{~L} / \mathrm{i} / \mathrm{A}}\end{array}$ & $\begin{array}{l}{[\mathrm{E}-144} \\
\mathrm{fCi} / \mathrm{in}]\end{array}$ \\
\hline $6 / 5-6 / 8$ & 195 & 0.034 & 0.139 & & & & & & 0.775 & & \\
\hline $6 / 8-b / 10$ & 367 & & & 0.06 & & & & & 0.120 & & \\
\hline $3 / 10-6 / 12$ & 200 & & & & & & & & 0.110 & & \\
\hline$b / 12-b / 15$ & 103 & & & & & & & & 0.214 & & \\
\hline $6 / 15-6 / 17$ & 95 & & & & & & & & 0.061 & & \\
\hline $6 / 17-6 / 17$ & 100 & & & & & & & & 0.047 & & \\
\hline $6 / 19-6 / 22$ & 121 & 0.027 & & & & & & & 0.185 & & \\
\hline $6 / 22-6 / 2 b$ & 104 & & & & & & & & 0.066 & & \\
\hline $6 / 26-6 / 25$ & 200 & & & & & & & & 0.081 & & \\
\hline 6/29-7/1/87 & 226 & & & & & & & & 0.142 & & \\
\hline $7 / 1-7 / 2$ & 103 & & & & & & & & 0.129 & & \\
\hline $7 / 2-7 / 7$ & 35 & & & & & & & & $-0.05 \mathrm{~s} 2$ & & \\
\hline $7 / 7-7 / 9$ & 181 & & 0.094 & & & & & & 0.086 & & \\
\hline $7 / 9-7 / 10$ & 126 & & & & & & & & -0.092 & & \\
\hline $7 / 10-7 / 13$ & 95 & & & & & & & & 0.033 & & \\
\hline $7 / 13-7 / 15$ & 172 & & & & & & & & -0.120 & 0.308 & \\
\hline $7 / 15-7 / 17$ & 228 & & 0.05 & & & & & & 0.064 & & \\
\hline $7 / 17-7 / 20$ & 225 & & & & & & & & 0.046 & & \\
\hline $7 / 20-7 / 22$ & 301 & 0.348 & & & & & & & 0.180 & & \\
\hline $7 / 22-7 / 24$ & 309 & & & & & & & & 0.078 & & \\
\hline $7 / 24-7 / 27$ & 174 & & & & & & & & 0.215 & & \\
\hline $7 / 27-7 / 29$ & 219 & & & & & & & & -0.220 & & \\
\hline $7 / 29-7 / 31$ & 159 & & & & & & & & 0.368 & & \\
\hline $7 / 31-8 / 3 / 67$ & 174 & & & & & & & & -0.052 & & \\
\hline $8 / 3-8 / 5$ & 126 & & & 0.03 & & & & & 0.041 & & \\
\hline $8 / 5-8 / 7$ & 140 & & & & & & & & 0.066 & & \\
\hline $8 / 7-8 / 10$ & 95 & & & & & & & & -0.060 & & \\
\hline $8 / 10-8 / 12$ & 129 & & & & & & & & 0.049 & & \\
\hline $8 / 12-8 / 14$ & 164 & & & & & & & & 0.049 & & \\
\hline $8 / 14-8 / 17$ & 141 & & & & & & & & 0.049 & & \\
\hline $8 / 17-8 / 19$ & 153 & & & & & & & & 0.091 & 0.074 & \\
\hline$B / 19-8 / 21$ & 162 & & & & & & & & 1.780 & & \\
\hline $8 / 21-8 / 24$ & 207 & & & & & & & & 0.773 & & \\
\hline $8 / 24-8 / 26$ & 159 & & & & & & & & 0.130 & & \\
\hline $8 / 26-8 / 28$ & 196 & & & & & & & & 0.173 & & \\
\hline $8 / 28-8 / 31$ & 165 & & & & & & & & 0.136 & & \\
\hline $9 / 31-9 / 2 / 87$ & 86 & & & & & & & & -0.033 & & \\
\hline $7 / 2-9 / 4$ & 134 & & & & & & & & -0.038 & & \\
\hline $9 / 4-9 / 8$ & 33 & & & & & & & & -0.040 & & \\
\hline $9 / 8-9 / 9$ & 164 & & & & & & & & -0.070 & & \\
\hline $9 / 9-7 / 11$ & 104 & & & & & & & & -0.037 & & \\
\hline $9 / 11-9 / 14$ & 83 & & & & & & & & 0.116 & & \\
\hline $9 / 14-9 / 16$ & 134 & & & & & & & & -0.040 & & \\
\hline $9 / 16-9 / 18$ & 178 & & & & & & & & 0.110 & & \\
\hline $9 / 18-9 / 21$ & 118 & & & & & & & & 0.124 & & \\
\hline $9 / 21-9 / 23$ & 179 & & & & & & & & 0.260 & & \\
\hline $9 / 23-9 / 25$ & 96 & & & & & & & & 0.349 & & \\
\hline $9 / 25-9 / 28$ & 169 & & & & & & & & 0.092 & & \\
\hline $9 / 20-7 / 30$ & 214 & & & & & & & & -0.053 & & \\
\hline $9 / 30-10 / 2 / 87$ & 134 & & & & & & & & 0.029 & & \\
\hline $10 / 2-10 / 5$ & 104 & & & & & & & & 0.146 & & \\
\hline $10 / 5-10 / 7$ & 202 & & & & & & & & 0.085 & & \\
\hline $10 / 7-10 / 9$ & 108 & & & & & & & & 0.072 & & \\
\hline
\end{tabular}




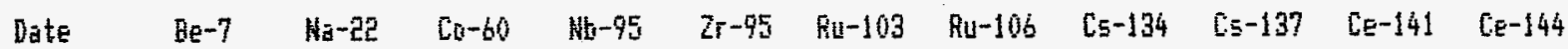

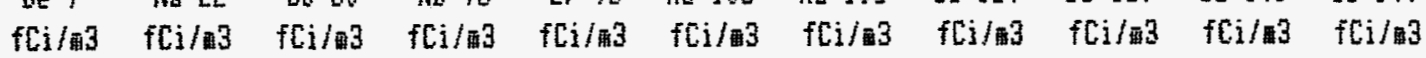

\begin{tabular}{|c|c|c|c|c|}
\hline $10 / 9-10 / 12$ & 155 & & & 0.185 \\
\hline $10 / 12-10 / 14$ & 153 & & & 0.103 \\
\hline $10 / 14-10 / 16$ & 209 & & & 0.153 \\
\hline $10 / 16-10 / 19$ & 203 & & & 0.213 \\
\hline $10 / 19-10 / 21$ & 210 & & & 0.258 \\
\hline $10 / 21-10 / 23$ & 112 & & 0.03 & 0.157 \\
\hline $10 / 23-10 / 26$ & 236 & & & 0.291 \\
\hline $10 / 26-10 / 28$ & 128 & & & 0.066 \\
\hline $10 / 28-10 / 30$ & 121 & & & 0.065 \\
\hline $10 / 30-11 / 2 / 87$ & 177 & & & 0.258 \\
\hline $11 / 2-11 / 4$ & 179 & & & 0.107 \\
\hline $11 / 4-11 / 6$ & 211 & & & 0.113 \\
\hline $11 / 6-11 / 9$ & 114 & & & 0.093 \\
\hline $11 / 9-11 / 11$ & 114 & & & 0.460 \\
\hline $11 / 11-11 / 13$ & 178 & & & 0.041 \\
\hline $11 / 13-11 / 16$ & 152 & & & 0.088 \\
\hline $11 / 16-11 / 18$ & $15 \mathrm{i}$ & & & 0.165 \\
\hline $11 / 18-11 / 20$ & 159 & 0.020 & & -0.019 \\
\hline $11 / 20-11 / 23$ & 252 & & & 0.281 \\
\hline $11 / 23-11 / 25$ & 195 & & & 3.400 \\
\hline $11 / 25-11 / 30$ & 82 & & & -0.027 \\
\hline $11 / 30-12 / 2 / 97$ & 的 & & & -0.033 \\
\hline $12 / 2-12 / 4$ & 161 & & & -0.048 \\
\hline $12 / 4-12 / 7$ & 95 & & & -0.046 \\
\hline $12 / 7-12 / 9$ & 137 & & & 0.036 \\
\hline $12 / 9-12 / 11$ & 179 & & & 0.058 \\
\hline $12 / 11-12 / 14$ & 123 & & & -0.036 \\
\hline $12 / 14-12 / 16$ & 145 & & & -0.033 \\
\hline $12 / 16-12 / 18$ & 129 & & & -0.059 \\
\hline $12 / 18-12 / 21$ & 171 & & & -0.023 \\
\hline $12 / 21-12 / 23$ & 96 & & & -0.046 \\
\hline $12 / 23-12 / 29$ & 90 & & & -0.022 \\
\hline $12 / 28-12 / 30$ & 106 & & & -0.049 \\
\hline $12 / 30-1 / 4 / 88$ & 143 & & & 0.132 \\
\hline $1 / 4-1 / 6$ & 230 & & & 0.262 \\
\hline $1 / 6-1 / 11$ & 117 & & 0.01 & 0.022 \\
\hline $1 / 11-1 / 13$ & 184 & & & 0.042 \\
\hline $1 / 13-1 / 15$ & 112 & & & -0.035 \\
\hline $1 / 15-1 / 18$ & 119 & & & -0.041 \\
\hline $1 / 18-1 / 20$ & 58 & & & 0.048 \\
\hline $1 / 20-1 / 22$ & 95 & & & -0.047 \\
\hline $1 / 2 \overline{2}-1 / 25$ & 97 & & & 0.057 \\
\hline $1 / 25-1 / 27$ & 98 & & & -0.030 \\
\hline $1 / 27-1 / 29$ & 97 & & & 0.058 \\
\hline $1 / 29-2 / 1 / 80$ & 72 & & & 0.052 \\
\hline $2 / 1-2 / 3$ & 93 & & & -0.056 \\
\hline $2 / 3-2 / 5$ & 41 & & & -0.024 \\
\hline $2 / 5-2 / 8$ & 198 & & & -0.030 \\
\hline $2 / 8-2 / 10$ & 192 & & & -0.039 \\
\hline $2 / 10-2 / 12$ & 115 & & & -0.053 \\
\hline $2 / 12-2 / 16$ & 121 & & 0.05 & -0.029 \\
\hline $2 / 16-2 / 19$ & 130 & 0.030 & & 0.038 \\
\hline 2/17-2/22 & 157 & & 0.06 & -0.038 \\
\hline
\end{tabular}




\begin{tabular}{|c|c|c|}
\hline $\begin{array}{c}\mathrm{Be}-7 \\
\mathrm{fCi} / \text { 醇 }\end{array}$ & 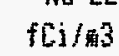 & $\mathrm{fCi} / \overline{\mathrm{m}}$ \\
\hline
\end{tabular}

\begin{tabular}{|c|c|c|c|c|}
\hline $2 / 22-2 / 24$ & 114 & & & -0.037 \\
\hline $2 / 24-2 / 26$ & 348 & & & 0.039 \\
\hline $2 / 26-2 / 29$ & 203 & & & -0.110 \\
\hline $2 / 29-3 / 2 / 8 \mathrm{~B}$ & 216 & 0.053 & & 0.055 \\
\hline $3 / 2-3 / 4$ & 206 & & & 0.146 \\
\hline $3 / 4-3 / 7$ & 84 & & & -0.031 \\
\hline $3 / 7-3 / 9$ & 152 & & & 0.039 \\
\hline $3 / 9-3 / 11$ & 41 & & & -0.033 \\
\hline $3 / 11-3 / 14$ & 162 & & & -0.025 \\
\hline $3 / 14-3 / 16$ & 151 & & & -0.033 \\
\hline $3 / 16-3 / 18$ & 84 & & & 0.062 \\
\hline $3 / 18-3 / 21$ & 134 & & & -0.037 \\
\hline $3 / 21-3 / 23$ & 203 & & & -0.180 \\
\hline $3 / 23-3 / \mathrm{E}^{5}$ & 91 & & & 0.065 \\
\hline $3 / 25-3 / 28$ & 161 & 0.028 & 0.02 & 0.056 \\
\hline $3 / 2 \theta-3 / 30$ & 256 & 0.037 & & -0.038 \\
\hline $3 / 30-3 / 31$ & 258 & & & -0.011 \\
\hline $3 / 31-4 / 4 / 88$ & 187 & & 0.02 & 0.103 \\
\hline $4 / 4-4 / 6$ & 184 & & & 0.112 \\
\hline $4 / 6-4 / 8$ & 83 & & 0.05 & 0.171 \\
\hline $4 / 8-4 / 11$ & 276 & 0.076 & 0.02 & 0.178 \\
\hline $4 / 11-4 / 13$ & 107 & & & 0.024 \\
\hline $4 / 13-4 / 15$ & 92 & & & 0.061 \\
\hline $4 / 15-4 / 18$ & 170 & & & 0.150 \\
\hline $4 / 18-4 / 20$ & 233 & & & 0.213 \\
\hline $4 / 20-4 / 22$ & 99 & & & 0.070 \\
\hline $4 / 22-4 / 25$ & 121 & & & -0.034 \\
\hline $4 / 25-4 / 27$ & 63 & & & -0.034 \\
\hline \multicolumn{5}{|l|}{$4 / 22^{-}$} \\
\hline $4 / 29-5 / 2 / 88$ & 208 & & & 0.092 \\
\hline $5 / 2-5 / 4$ & 169 & & & 0.051 \\
\hline $5 / 4-5 / 6$ & 144 & 0.038 & & -0.034 \\
\hline $5 / 6-5 / 9$ & 130 & & & 0.064 \\
\hline $5 / 9-5 / 11$ & 154 & & & -0.059 \\
\hline $5 / 11-5 / 13$ & 161 & & & 0.046 \\
\hline $5 / 13-5 / 16$ & 154 & & & 0.392 \\
\hline $5 / 16-5 / 18$ & 157 & & & -0.038 \\
\hline $5 / 18-5 / 20$ & 255 & & & 0.177 \\
\hline $5 / 20-5 / 23$ & 127 & & & $0.04 \varepsilon$ \\
\hline $5 / 23-5 / 25$ & 132 & & & -0.043 \\
\hline $5 / 25-5 / 27$ & 145 & & & -0.067 \\
\hline $5 / 27-5 / 31$ & 178 & & & 0.143 \\
\hline $5 / 31-6 / 3 / 88$ & 246 & & & 0.078 \\
\hline$b / 3-b / 6$ & 123 & & & 0.101 \\
\hline $6 / 6-6 / 8$ & 118 & & & 0.135 \\
\hline $6 / 8-6 / 10$ & 130 & & & 0.095 \\
\hline $6 / 10-6 / 14$ & 215 & 0.026 & & 0.055 \\
\hline $6 / 14-6 / 17$ & $2 \pm 2$ & 0.033 & & 0.117 \\
\hline $6 / 17-6 / 22$ & 183 & & & 0.109 \\
\hline $6 / 22-6 / 24$ & 193 & & & 0.094 \\
\hline $6 / 24-6 / 27$ & 165 & & & 0.069 \\
\hline $6 / 27-6 / 29$ & 128 & & 0.02 & 0.259 \\
\hline $6 / 29-7 / 5 / 88$ & 156 & & & 0.147 \\
\hline
\end{tabular}




\begin{tabular}{|c|c|c|c|c|c|c|c|c|c|c|c|}
\hline Date & $\begin{array}{l}\mathrm{HE}-7 \\
\mathrm{fCi} / \mathrm{m} 3\end{array}$ & 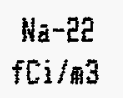 & $\begin{array}{c}C 0-60 \\
\text { fCi//醇 }\end{array}$ & $\begin{array}{l}\text { Wh- }-95 \\
\mathrm{fCi} / \mathrm{i} / \mathrm{T}\end{array}$ & $\begin{array}{r}2 \mathrm{r}-95 \\
\mathrm{fCi} / \mathrm{m} 3\end{array}$ & $\begin{array}{l}\mathrm{Fu}-103 \\
\mathrm{fCi} / \text { I }^{-3} 3\end{array}$ & $\begin{array}{l}\text { Ru-106 } \\
\mathrm{fCi} / \mathrm{A} \text { 的 }\end{array}$ & $\begin{array}{l}C_{5}-134 \\
\mathrm{fCi} / \mathrm{I}^{3} 3\end{array}$ & $\begin{array}{l}{[5-137} \\
f C 1 / 43\end{array}$ & 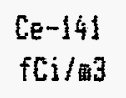 & $\begin{array}{l}{[\mathrm{E}-144} \\
\mathrm{f}[\mathrm{Ci} / \mathrm{m}\end{array}$ \\
\hline $7 / 5-5 / 8$ & 218 & & & & & & & & 0.168 & & \\
\hline $7 / 8-7 / 11$ & 177 & & & & 0.02 & & & & 0.064 & & \\
\hline $7 / 11-7 / 13$ & 72 & & & & & & & & 0.122 & & \\
\hline $7 / 13-7 / 15$ & 93 & & & & & & & & 0.110 & & \\
\hline $7 / 15-7 / 18$ & 101 & & & & & & & & 0.120 & & \\
\hline $7 / 18-7 / 20$ & 116 & & & & & & & & 0.081 & & \\
\hline $7 / 20-7 / 22$ & 82 & & & & & & & & 0.054 & & \\
\hline \multicolumn{12}{|l|}{$7 / 27-$} \\
\hline $7 / 25-7 / 27$ & 225 & & & & & & & & 0.066 & & \\
\hline $7 / 27-7 / 29$ & 141 & & & & & & & & 0.067 & & \\
\hline $7 / 29-8 / 1 / 88$ & 138 & & & & 0.04 & & & & 0.044 & & \\
\hline $8 / 1-8 / 3$ & 134 & & & & 0.06 & & & & 0.065 & & \\
\hline $8 / 3-8 / 5$ & 122 & & & & & & & & -0.046 & & \\
\hline $8 / 5-8 / 6$ & 98 & & & & & & & & -0.033 & & \\
\hline $6 / 8-8 / 10$ & 119 & & & & & & & & 0.047 & & \\
\hline $8 / 10-8 / 12$ & 95 & & & & & & & & 0.091 & & \\
\hline $8 / 12-8 / 15$ & 107 & & & & & & & & 0.028 & & \\
\hline $8 / 15-8 / 17$ & 63 & & & & & & & & -0.061 & & \\
\hline $8 / 17-8 / 19$ & 88 & & & 0.033 & & & & & 0.055 & & \\
\hline $8 / 19-8 / 22$ & 92 & & & & & & & & 0.158 & & \\
\hline $8 / 22-8 / 24$ & 94 & & & & & & & & -0.061 & & \\
\hline $8 / 24-8 / 26$ & 186 & & & & & & & & -0.055 & & \\
\hline $8 / 26-8 / 29$ & 129 & & & & & & & & -0.069 & & \\
\hline \multirow{2}{*}{\multicolumn{12}{|c|}{$\begin{array}{l}\text { 9/29- } \\
9 / 2-186\end{array}$}} \\
\hline & & & & & & & & & & & \\
\hline \multicolumn{12}{|l|}{$9 / 4-$} \\
\hline \multicolumn{12}{|l|}{$9 / 6-$} \\
\hline \multicolumn{12}{|l|}{$9 / 8-$} \\
\hline \multicolumn{12}{|l|}{$9 / 10^{-}$} \\
\hline \multicolumn{12}{|l|}{$9 / 12-$} \\
\hline \multicolumn{12}{|l|}{$9 / 14-$} \\
\hline \multicolumn{12}{|l|}{$9 / 16-$} \\
\hline \multirow{2}{*}{\multicolumn{12}{|c|}{ Ho samples cullected fru $8 / 29 / 80$ to $10 / 12 / 88$}} \\
\hline & & & & & & & & & & & \\
\hline \multicolumn{12}{|l|}{ 9/22- } \\
\hline \multicolumn{12}{|l|}{$9 / 24-$} \\
\hline \multicolumn{12}{|l|}{$9 / 26-$} \\
\hline \multicolumn{12}{|l|}{$9 / 28-$} \\
\hline \multicolumn{12}{|l|}{$7 / 30-$} \\
\hline \multicolumn{12}{|l|}{$10 / 1-18$ 日 } \\
\hline $10 / 2-$ & & & & & & & & & & & \\
\hline $10 / 4-$ & & & & & & & & & & & \\
\hline $10 / 6-$ & & & & & & & & & & & \\
\hline $10 / 8-$ & & & & & & & & & & & \\
\hline $10 / 10-$ & & & & & & & & & & & \\
\hline $10 / 12-10 / 14$ & 109 & & & & & & & & 0.030 & & \\
\hline $10 / 14-10 / 17$ & 143 & & & & & & & & 0.043 & & \\
\hline $50 / 17-10 / 19$ & 155 & & & & & & & & 0.149 & & \\
\hline $10 / 19-10 / 21$ & 121 & & & & & & & & 0.026 & & \\
\hline $10 / 21-10 / 24$ & 148 & 0.011 & & & & & & & 0.015 & & \\
\hline $10 / 24-10 / 26$ & 150 & & & & & & & & 0.028 & & \\
\hline $10 / 26-10 / 28$ & 118 & & & & & & & & -0.037 & & \\
\hline $10 / 28-10 / 31$ & 158 & & & & & & & & 0.020 & & \\
\hline
\end{tabular}




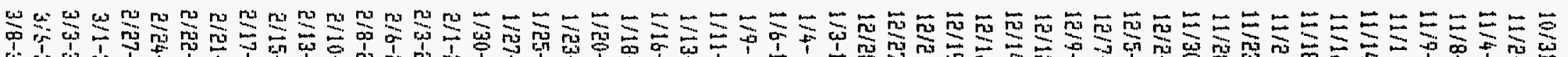

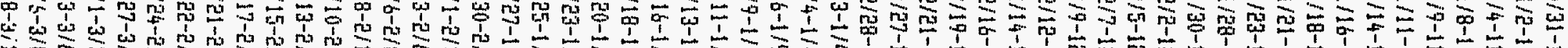

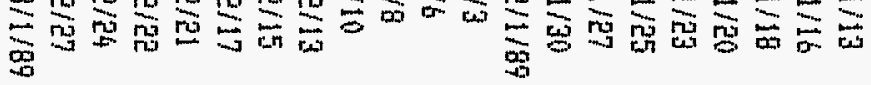

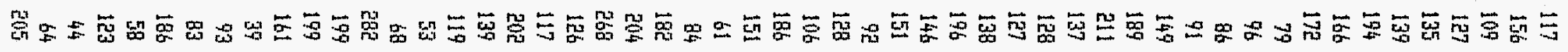

兽

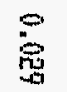

$\stackrel{\circ}{\Xi}$

空密

롤

害

意量

总

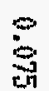

害点

总逽

空害

密密

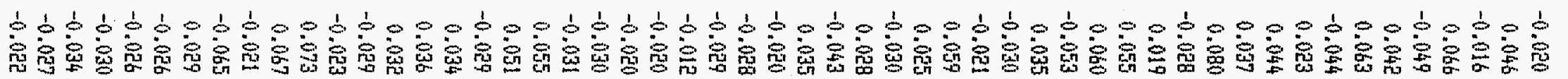
密要 


\begin{tabular}{|c|c|c|c|c|c|c|c|c|c|c|c|}
\hline Date & $\begin{array}{c}\mathrm{Be}-7 \\
\mathrm{fCi} / \mathrm{i} 3\end{array}$ & $\begin{array}{l}\mathrm{Aa}-2 \mathrm{2e} \\
\mathrm{fCi} / \mathrm{A} 3\end{array}$ & 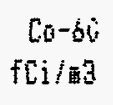 & 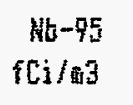 & $\begin{array}{r}2 r-95 \\
\mathrm{fCi} / 1 / 3\end{array}$ & $\begin{array}{l}R u-103 \\
\text { fTi } / \text { T. }\end{array}$ & 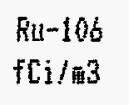 & $\begin{array}{l}{[5-134} \\
f[\mathrm{i} / \mathrm{w} 3\end{array}$ & $\begin{array}{l}\mathrm{Cs}-137 \\
\mathrm{fCi} / \mathrm{m}\end{array}$ & $\begin{array}{l}\mathrm{Ce}-14] \\
\mathrm{CLi} / \mathrm{m}\end{array}$ & 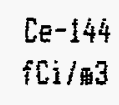 \\
\hline $3 / 10-3 / 13$ & 232 & 0.017 & & & & & & & 0.027 & & \\
\hline $3 / 13-3 / 15$ & 193 & & & & & & & & 0.057 & & \\
\hline $3 / 15-3 / 17$ & 177 & & & & & & & & 0.073 & & \\
\hline $3 / 17-3 / 20$ & 136 & 0.014 & & & & & & & 0.053 & & \\
\hline $3 / 20-3 / \mathrm{Ez}$ & 120 & & & & & & & & 0.069 & & \\
\hline $3 / 22-3 / 23$ & 125 & & & & & & & & -0.049 & & \\
\hline $3 / 23-3 / 27$ & 43 & & & & & & & & 0.042 & & \\
\hline $3 / 27-3 / 27$ & 127 & & & & & & & & 0.660 & & \\
\hline $3 / 29-3 / 31$ & 178 & & & & & & & & 0.048 & & \\
\hline $3 / 31-4 / 3 / 89$ & 87 & & & & & & & & 0.036 & & \\
\hline $4 / 3-4 / 5$ & 177 & 0.032 & & & & & & & 0.041 & & \\
\hline $4 / 5-4 / 7$ & $12 ?$ & & & & & & & & 0.098 & & \\
\hline $4 / 7-4 / 10$ & 113 & & & & & & & & -0.025 & & \\
\hline $4 / 10-4 / 12$ & 230 & 0.022 & & & & & & & 0.060 & & \\
\hline $4 / 12-4 / 14$ & 152 & & & & & & & & -0.017 & & \\
\hline $4 / 14-4 / 17$ & 73 & & & & & & & & -0.018 & & \\
\hline $4 / 17-4 / 19$ & 241 & & & & & & & & 0.072 & & \\
\hline $4 / 19-4 / 21$ & 208 & & & & & & & & 0.042 & & \\
\hline $4 / 21-4 / 24$ & 226 & 0.035 & & & & & & & 0.037 & & \\
\hline $4 / 24-4 / 26$ & 230 & & & & & & & & 0.056 & & \\
\hline $4 / 2 b-4 / 28$ & 284 & 0.048 & & & & & & & 0.058 & & \\
\hline $4 / 28-5 / 1 / 89$ & 197 & & & & & & & & 0.047 & & \\
\hline $5 / 1-5 / 3$ & 133 & & & & & & & & -0.038 & & \\
\hline $5 / 3-5 / 5$ & 203 & 0.032 & & & & & & & -0.029 & & \\
\hline $5 / 5-5 / 8$ & 129 & & & & & & & & 0.068 & & \\
\hline $5 / 8-5 / 10$ & 115 & 0.030 & & & & & & & -0.027 & & \\
\hline $5 / 10-5 / 12$ & 67 & & & & & & & & -0.030 & & \\
\hline $5 / 12-5 / 15$ & 175 & 0.024 & & & & & & & 0.025 & & \\
\hline $5 / 15-$ & & & & & & & & & & & \\
\hline $5 / 17-5 / 19$ & 81 & & & & & & & & 0.037 & & \\
\hline $5 / 19-5 / 21$ & 130 & & & & & & & & 0.032 & & \\
\hline $5 / 21-5 / 2^{4}$ & 119 & & & & & & & & -0.030 & & \\
\hline $5 / 24-5 / 26$ & 176 & & & & & & & & 0.084 & & \\
\hline $5 / 26-5 / 30$ & 119 & & & & & & & & -0.024 & & \\
\hline $5 / 30-5 / 31$ & 142 & & & & & & & & 0.034 & & \\
\hline $5 / 31-6 / 2 / 8 ?$ & 131 & & & & & & & & 0.048 & & \\
\hline $6 / 2-6 / 5$ & 117 & & & & & & & & 0.092 & & \\
\hline $6 / 5-6 / 7$ & 83 & & & 0.02 & & & & & -0.021 & & \\
\hline $6 / 7-6 / 9$ & 75 & 0.016 & & & & & & & -0.028 & & \\
\hline$b / 9-b / 12$ & 127 & & & & & & & & 0.016 & & \\
\hline $6 / 12-6 / 14$ & 102 & & & & & & & & 0.104 & & \\
\hline$b / 14-b / 1 b$ & 101 & & & & & & & & 0.050 & & \\
\hline $6 / 16-6 / 19$ & 96 & & & & & & & & 0.052 & & \\
\hline $6 / 19-6 / 21$ & 80 & & & & & & & & -0.026 & & \\
\hline $6 / 21-6 / 23$ & 97 & & & & & & & & -0.025 & & \\
\hline 6/23-6/26 & 132 & 0.016 & & & & & & & 0.020 & & \\
\hline$b / 2 b-6 / 20$ & 123 & & & & & & & & 0.052 & & \\
\hline $6 / 28-6 / 30$ & 143 & & & & & & & & 0.070 & & \\
\hline $6 / 30-7 / 5 / 89$ & 143 & 0.023 & & & & & & & 0.099 & & \\
\hline $7 / 5-7 / 7$ & 93 & & & & & & & & 0.045 & & \\
\hline $7 / 7-7 / 10$ & 137 & & & & & & & & 0.018 & & \\
\hline $7 / 10-7 / 12$ & 124 & & & & & & & & 0.048 & & \\
\hline $7 / 1 \overline{\mathrm{e}}-7 / 14$ & 89 & & & & & & & & -0.019 & & \\
\hline
\end{tabular}




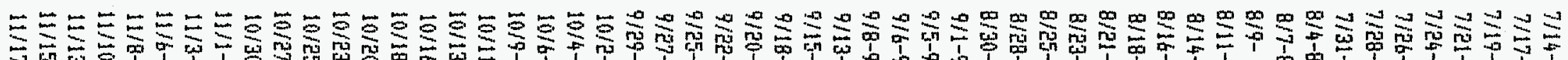

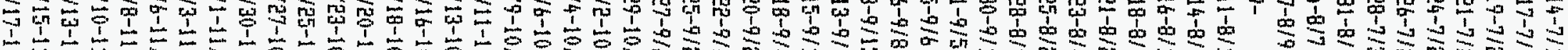

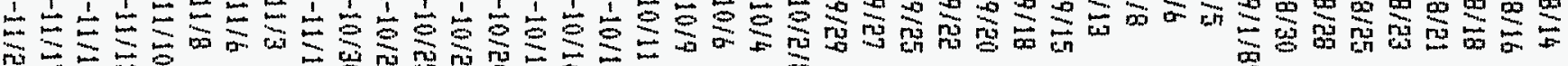

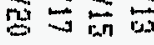
$\underbrace{}_{-\infty}$

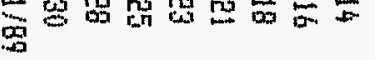

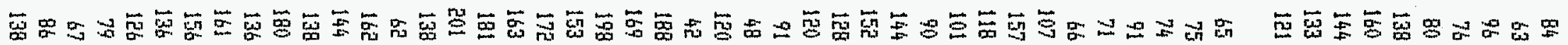

突

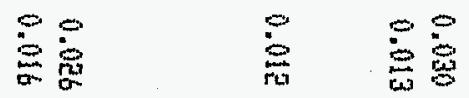

产

承

蛋蛋

:

总暑

范票

密点

8

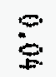

密

蛞害

总要

苛

害要

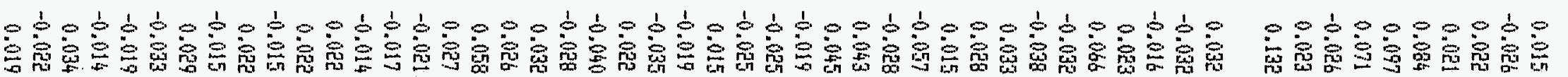
密要畜 害密 空 


\begin{tabular}{|c|c|c|c|c|c|c|c|c|c|c|}
\hline Date & 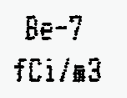 & 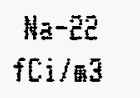 & 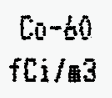 & 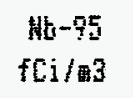 & 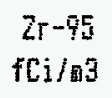 & 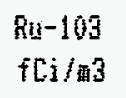 & $\begin{array}{l}R u-106 \\
\text { fCi } /-\overline{6} 3\end{array}$ & $\begin{array}{l}{[\mathrm{c}-134} \\
\{\mathrm{fi} / \mathrm{i} / \mathrm{S}\}\end{array}$ & 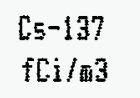 & $\begin{array}{l}{[\mathrm{E}-141} \\
\mathrm{fCi} / \mathrm{m}]\end{array}$ \\
\hline $11 / 20-11 / 22$ & 175 & 0.021 & & & & & & & -0.020 & \\
\hline $11 / 22-11 / 27$ & 124 & $0.01 \bar{z}$ & & & & & & & 0.019 & \\
\hline $11 / 27-11 / 29$ & 101 & & & & & & & & -0.022 & \\
\hline $11 / 29-12 / 1 / 89$ & 91 & & & & & & & & -0.013 & \\
\hline $12 / 1-12 / 4$ & 100 & & & & & & & & 0.015 & \\
\hline $12 / 4-12 / 6$ & 198 & & & & & & & & -0.024 & \\
\hline $12 / 6-12 / 8$ & 84 & & & & & & & & -0.027 & \\
\hline $12 / 8-12 / 11$ & 53 & & & & & & & & -0.025 & \\
\hline $12 / 11-12 / 13$ & 63 & & & & & & & & -0.028 & \\
\hline $12 / 13-12 / 15$ & 136 & & & & & & & & -0.041 & \\
\hline $12 / 15-12 / 18$ & 156 & & & & & & & & -0.027 & \\
\hline $12 / 18-12 / 20$ & 珀 & & & & & & & & -0.025 & \\
\hline $12 / 20)-12 / 22$ & 137 & & & & & & & & -0.046 & \\
\hline $12 / 22-12 / 27$ & 97 & & & & & & & & -0.020 & \\
\hline $12 / 27-12 / 29$ & 50 & & & & & & & & -0.042 & \\
\hline $12 / 29-1 / 2 / 90$ & 110 & & & & & & & & 0.025 & \\
\hline $1 / 2-1 / 3$ & 100 & & & & & & & & -0.031 & \\
\hline $1 / 3-1 / 5$ & 189 & & & & & & & & -0.027 & \\
\hline $1 / 5-1 / 8$ & 78 & & & & & & & & -0.018 & \\
\hline $1 / 8-1 / 10$ & 100 & & & & & & & & -0.017 & \\
\hline $1 / 10-1 / 12$ & 114 & & & & & & & & -0.024 & \\
\hline $1 / 12-1 / 15$ & 98 & & & & & & & & 0.045 & \\
\hline $1 / 15-1 / 17$ & 197 & & & & & & & & 0.064 & \\
\hline $1 / 17-1 / 19$ & 136 & & & & & & & & 0.050 & \\
\hline $1 / 19-1 / 2 E$ & 91 & & & & & & & & 0.030 & \\
\hline $1 / 22-1 / 24$ & 120 & & & & & & & & 0.025 & \\
\hline $1 / 24-1 / 26$ & 109 & & & & & & & & -0.044 & \\
\hline $1 / 26-1 / 29$ & 147 & & & & & & & & -0.021 & \\
\hline $1 / 29-1 / 31$ & 49 & & & & & & & & -0.010 & \\
\hline $1 / 31-2 / 2 / 90$ & 188 & & & & & & & & 0.030 & \\
\hline $2 / 2-2 / 5$ & 73 & & & & & & & & -0.025 & \\
\hline $2 / 5-2 / 7$ & 141 & & & & & & & & -0.042 & \\
\hline $2 / 7-2 / 9$ & 184 & 0.042 & & & & & & & -0.031 & \\
\hline $2 / 9-2 / 12$ & 123 & 0.020 & & & & & & & 0.043 & \\
\hline $2 / 12-2 / 14$ & 160 & & & & & & & & 0.060 & \\
\hline $2 / 14-2 / 16$ & 216 & & & & & & & & 0.059 & \\
\hline $2 / 16-2 / 20$ & 85 & & & & & & & & -0.014 & \\
\hline $2 / 20-2 / 21$ & 144 & & & & & & & & -0.039 & \\
\hline $2 / 21-2 / 23$ & 178 & & & & & & & & -0.023 & \\
\hline $2 / 23-2 / 26$ & 51 & & & & & & & & -0.058 & \\
\hline $2 / 2 b-3 / 1 / 90$ & 187 & & & & & & & & 0.037 & \\
\hline $3 / 1-3 / 2$ & 159 & & & & & & & & 0.032 & \\
\hline $3 / 2-3 / 5$ & 98 & & & & & & & & 0.031 & \\
\hline $3 / 5-3 / 7$ & 166 & & & & & & & & 0.034 & \\
\hline $3 / 7-3 / 9$ & 157 & & & & & & & & 0.047 & \\
\hline $3 / 9-3 / 12$ & 129 & & & & & & & & 0.100 & \\
\hline $3 / 12-3 / 14$ & 128 & & & & & & & & 0.192 & \\
\hline $3 / 14-3 / 16$ & 103 & & & 0.03 & & & & & 0.132 & \\
\hline $3 / 1 t-3 / 19$ & 90 & & & & & & & & 0.060 & \\
\hline $3 / 19-3 / 21$ & 158 & & & & & & & & 0.024 & \\
\hline $3 / 21-3 / 23$ & 244 & 0.022 & & & & & & & 0.044 & \\
\hline $3 / 23-3 / 26$ & 197 & & & 0.01 & & & & & 0.032 & \\
\hline $3 / 26-3 / 28$ & 133 & & & & & & & & 0.024 & \\
\hline
\end{tabular}




\begin{tabular}{|c|c|c|c|c|c|c|c|c|c|c|}
\hline Date & $\begin{array}{c}\mathrm{Be}-7 \\
\mathrm{fCl} / \mathrm{Ag}\end{array}$ & 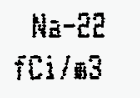 & $\begin{array}{r}{[0-60} \\
\{[\mathrm{i} / \mathrm{i}-\mathrm{s}\end{array}$ & 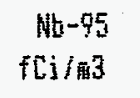 & $\begin{array}{r}2 r-95 \\
\mathrm{fCi} / \text { 俰 }\end{array}$ & $\begin{array}{l}\text { Ru-103 } \\
\mathrm{iC \textrm {i } / \mathrm { II }}\end{array}$ & $\begin{array}{l}\text { Fu- } 106 \\
\mathrm{fCi} / \text { F }^{3} 3\end{array}$ & $\begin{array}{l}C_{5}-134 \\
\mathrm{fCi} / \mathrm{F}^{3}\end{array}$ & 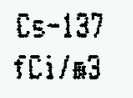 & $\begin{array}{l}{[E-14]} \\
{[C \mathrm{i} / \mathrm{E}]}\end{array}$ \\
\hline $3 / 29-3 / 30$ & 106 & & & & & & & & -0.011 & \\
\hline $3 / 30-4 / 2 / 90$ & 57 & & & & & & & & 0.014 & \\
\hline $4 / 2-4 / 4$ & 88 & & & 0.01 & & & & & -0.016 & \\
\hline $4 / 4-4 / 9$ & 173 & 0.012 & & & & & & & 0.021 & \\
\hline $4 / 9-4 / 11$ & 174 & 0.039 & & & & & & & $0.02 b$ & \\
\hline $4 / 11-4 / 12$ & 109 & & & & & & & & -0.028 & \\
\hline $4 / 12-4 / 16$ & 161 & 0.030 & & & & & & & 0.022 & \\
\hline $4 / 16-4 / 18$ & 156 & 0.034 & & & & & & & 0.025 & \\
\hline $4 / 18-4 / 20$ & 210 & & & & & & & & 0.048 & \\
\hline $4 / 20-4 / 23$ & 144 & 0.019 & & & & & & & 0.032 & \\
\hline $4 / 23-4 / 25$ & 162 & 0.019 & & & & & & & 0.035 & \\
\hline $4 / 25-4 / 27$ & 182 & & & & & & & & 0.158 & \\
\hline $4 / 27-4 / 30$ & 168 & & & & & & & & 0.030 & \\
\hline $4 / 30-5 / 2 / 90$ & 114 & & & & & & & & -0.018 & \\
\hline $5 / 2-5 / 4$ & 129 & & & & & & & & $0.08 \mathrm{z}$ & \\
\hline $5 / 4-5 / 7$ & 78 & & & & & & & & 0.087 & \\
\hline $5 / 7-5 / 0$ & 172 & & & & & & & & -0.038 & \\
\hline $5 / 8-5 / 9$ & 144 & & & & & & & & -0.052 & \\
\hline $5 / 9-5 / 11$ & 141 & 0.042 & & & & & & & -0.088 & \\
\hline $5 / 11-5 / 14$ & 194 & 0.029 & & 0.01 & & & & & $0.0 \mathrm{e}$ & \\
\hline $5 / 14-5 / 16$ & 176 & 0.021 & & & & & & & 0.056 & \\
\hline $5 / 16-5 / 18$ & 133 & & & & & & & & 0.029 & \\
\hline $5 / 19-5 / 21$ & 131 & & & & & & & & 0.032 & \\
\hline $5 / 21-5 / 23$ & 62 & & & & & & & & -0.022 & \\
\hline $5 / 23-5 / 25$ & 89 & & & & & & & & 0.026 & \\
\hline $5 / 25-5 / 29$ & 105 & 0.013 & & & & & & & 0.021 & \\
\hline $5 / 29-5 / 31$ & 116 & & & & & & & & -0.015 & \\
\hline $5 / 31-6 / 1 / 90$ & 76 & & & & & & & & -0.049 & \\
\hline $6 / 1-6 / 4$ & 115 & 0.016 & & 0.020 & & & & & 0.019 & \\
\hline $6 / 4-6 / 6$ & 90 & 0.027 & & & & & & & -0.019 & \\
\hline $6 / 6-6 / 9$ & 107 & & & & & & & & 0.043 & \\
\hline $6 / 9-6 / 12$ & 36 & & & & & & & & -0.040 & \\
\hline $6 / 12-6 / 13$ & 99 & & & & & & & & -0.036 & \\
\hline $6 / 13-6 / 15$ & 110 & & & & & & & & 0.057 & \\
\hline $6 / 15-6 / 18$ & 78 & & & & & & & & 0.019 & \\
\hline $6 / 18-6 / 20$ & 107 & & & & & & & & 0.022 & \\
\hline $6 / 20-6 / 22$ & 135 & & & & & & & & 0.045 & \\
\hline $6 / 22-6 / 25$ & 116 & & & & & & & & -0.018 & \\
\hline $6 / 25-6 / 27$ & 107 & & & & & & & & 0.024 & \\
\hline $6 / 27-6 / 29$ & 116 & & & & & & & & -0.043 & \\
\hline $6 / 29-7 / 2 / 90$ & 135 & & & & & & & & 0.038 & \\
\hline $7 / 2-7 / 3$ & 102 & & & & & & & & 0.045 & \\
\hline $7 / 3-7 / 6$ & 97 & & & & & & & & 0.022 & \\
\hline $7 / 6-7 / 11$ & 127 & & & & & & & & -0.032 & \\
\hline $7 / 11-7 / 13$ & 103 & & & & & & & & -0.052 & \\
\hline $7 / 13-7 / 16$ & $9 \mathrm{~h}$ & & & & & & & & 0.025 & \\
\hline $7 / 1 b-7 / 18$ & 106 & & & 0.01 & & & & & -0.018 & \\
\hline $7 / 18-7 / 20$ & 55 & & & 0.04 & & & & & -0.031 & \\
\hline $7 / 20-7 / 23$ & 71 & & & & & & & & 0.071 & \\
\hline 7/23-7/25 & 71 & & & & & & & & -0.020 & \\
\hline 7/25-7/E7 & 99 & & & & & & & & -0.014 & \\
\hline $7 / 27-7 / 30$ & 94 & & & & & & & & 0.030 & \\
\hline $7 / 30-8 / 1 / 90$ & 127 & & & & & & & & 0.034 & \\
\hline
\end{tabular}




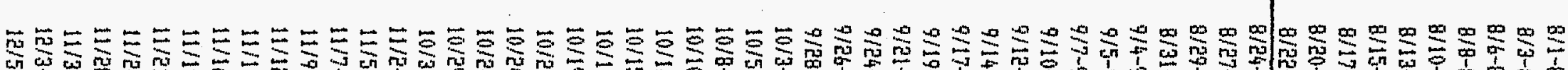

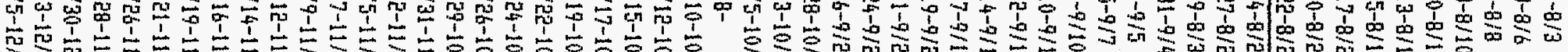

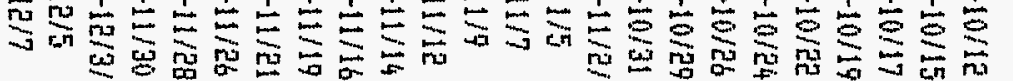

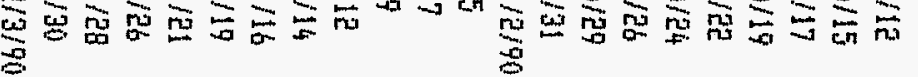

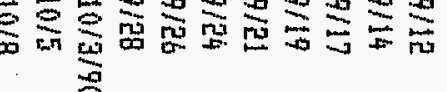

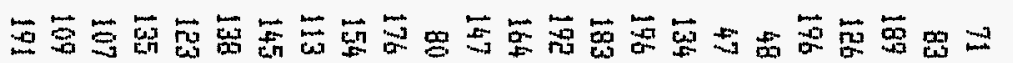

$\stackrel{8}{\overparen{G}}$

$\stackrel{8}{0}$

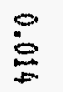

$\sum_{0 \rightarrow 1}^{0}$

量

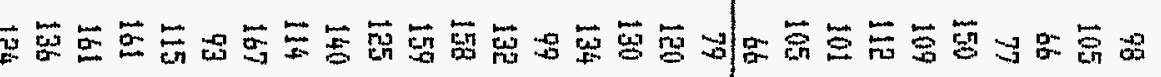

量

$\stackrel{\circ}{8} \stackrel{\circ}{\circ}$

융

:

要然

焉罟

혼 중

善蛊

离

灾

空

要步

蟔空

点璋

窇空

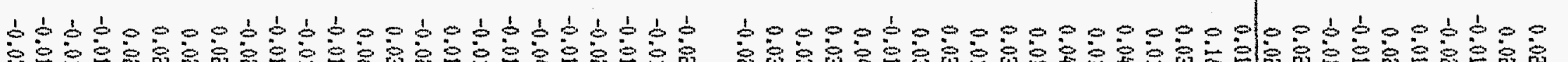

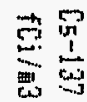
害营 


\begin{tabular}{|c|c|c|c|c|c|c|c|c|c|c|}
\hline & & $\begin{array}{l}\mathrm{Na}- \\
\mathrm{fEi}\end{array}$ & $\begin{array}{l}C \bar{a}-60 \\
f[i / m s\end{array}$ & $\begin{array}{l}\text { Hb-95 } \\
\mathrm{fCi} / \text { - }^{3}\end{array}$ & $\begin{array}{r}2 \mathrm{~T}-95 \\
\mathrm{fCi} / \mathrm{E}\end{array}$ & & & & & $\begin{array}{l}\text { Ce-141 } \\
\text { fCi } / \mathrm{E} 3\end{array}$ \\
\hline
\end{tabular}

\begin{tabular}{|c|c|c|c|c|}
\hline $1 \mathrm{e} / 7-1 \mathrm{e} / 10$ & 140 & & & -0.017 \\
\hline $12 / 10-12 / 12$ & 97 & & & -0.018 \\
\hline $12 / 12-12 / 14$ & 110 & & & -0.030 \\
\hline $12 / 14-12 / 17$ & 141 & & 0.02 & -0.017 \\
\hline $12 / 17-12 / 19$ & 117 & & & 0.032 \\
\hline $12 / 19-12 / 21$ & 61 & & & -0.016 \\
\hline $12 / 21-12 / 26$ & 29 & & & -0.068 \\
\hline $12 / 2 b-12 / 29$ & 110 & & & -0.025 \\
\hline $12 / 27-12 / 31$ & 31 & & & -0.033 \\
\hline $12 / 31-1 / 2 / 41$ & 120 & & & -0.039 \\
\hline $1 / 2-1 / 4$ & 87 & & & -0.028 \\
\hline $1 / 4-1 / 7$ & 190 & & 0.02 & 0.026 \\
\hline $1 / 7-1 / 9$ & 20 & & & -0.013 \\
\hline $1 / 9-1 / 11$ & 15 & & & -0.025 \\
\hline $1 / 11-1 / 14$ & 19 & & & $-0.01 \overline{2}$ \\
\hline $1 / 14-1 / 16$ & 95 & & & -0.019 \\
\hline $1 / 16-1 / 18$ & 68 & & & -0.016 \\
\hline $1 / 18-1 / 21$ & 53 & & & -0.013 \\
\hline $1 / 21-1 / 23$ & 52 & & & -0.018 \\
\hline $1 / 23-1 / 25$ & 102 & 0.019 & & -0.023 \\
\hline $1 / 25-1 / 29$ & 129 & & & -0.014 \\
\hline $1 / 29-1 / 30$ & 43 & & & -0.036 \\
\hline $1 / 30-3 / 1 / 91$ & 111 & & & -0.015 \\
\hline $2 / 1-2 / 4$ & 196 & 0.020 & & -0.013 \\
\hline $2 / 4-\tilde{c} / 6$ & 220 & & & 0.023 \\
\hline $2 / 6-2 / 8$ & 92 & & & -0.013 \\
\hline $2 / 8-2 / 11$ & 41 & & & -0.010 \\
\hline $2 / 11-2 / 13$ & 188 & & & 0.030 \\
\hline $2 / 13-2 / 15$ & 163 & & & -0.027 \\
\hline $2 / 15-2 / 19$ & 182 & 0.020 & & 0.038 \\
\hline $2 / 19-2 / 20$ & 173 & & & 0.074 \\
\hline $2 / 20-2 / 22$ & 101 & & & -0.027 \\
\hline $2 / 22-3 / 25$ & 101 & & 0.046 & -0.018 \\
\hline $2 / 25-2 / 27$ & 129 & & & -0.025 \\
\hline $2 / 27-3 / 1 / 91$ & 202 & & & 0.073 \\
\hline $3 / 1-3 / 4$ & 123 & & 0.016 & -0.013 \\
\hline $3 / 4-3 / 6$ & $9 \dot{b}$ & & & -0.019 \\
\hline $3 / 6-3 / 8$ & 138 & & & 0.050 \\
\hline $3 / 8-3 / 11$ & 128 & 0.016 & & 0.020 \\
\hline $3 / 11-3 / 13$ & 131 & 0.028 & 0.09 & -0.029 \\
\hline $3 / 13-3 / 15$ & 67 & & & -0.015 \\
\hline $3 / 15-3 / 18$ & 84 & & & 0.014 \\
\hline $3 / 18-3 / 20$ & 116 & 0.027 & & -0.026 \\
\hline $3 / 20-3 / 22$ & 156 & & & $0.04 \theta$ \\
\hline $3 / 22-3 / 25$ & 176 & & & 0.149 \\
\hline $3 / 25-3 / 27$ & 178 & & & 0.140 \\
\hline $3 / 27-3 / 28$ & 195 & & & 0.106 \\
\hline $3 / 28-4 / 1 / 91$ & 103 & & & 0.029 \\
\hline $4 / 1-4 / 3$ & 120 & & & 0.034 \\
\hline $4 / 3-4 / 5$ & 177 & & & 0.030 \\
\hline $4 / 5-4 / 8$ & 92 & & & 0.027 \\
\hline $4 / 8-4 / 10$ & 102 & & & 0.021 \\
\hline $4 / 10-4 / 12$ & $21 \%$ & & & 0.016 \\
\hline
\end{tabular}




\begin{tabular}{|c|c|c|c|c|c|c|c|c|c|c|c|}
\hline Date & $\begin{array}{c}B E-7 \\
f[j / 63\end{array}$ & $\begin{array}{r}\mathrm{Na}-2 \mathrm{Z} \\
\mathrm{fCi} /{ }_{-3} 3\end{array}$ & $\begin{array}{r}C a-60 \\
+5 i / 43\end{array}$ & $\begin{array}{r}\mu b-95 \\
5 \mathrm{C} i / \text { 而 } 3\end{array}$ & $\begin{array}{l}2 \mathrm{~T}-95 \\
\mathrm{FCH} / \overline{4} 3\end{array}$ & $\begin{array}{l}8 \mathrm{xu}-103 \\
\mathrm{fCi} / \mathrm{T} 3\end{array}$ & $\begin{array}{l}\mathrm{Ru}-106 \\
\mathrm{~F}[\mathrm{~S} / \overline{\mathrm{M}} 3\end{array}$ & $\begin{array}{l}{[5-134} \\
\mathrm{fCi} / \mathbf{3} 3\end{array}$ & $\begin{array}{l}\text { C5-137 } \\
\{[\mathrm{C}=3 \mathrm{~F}\end{array}$ & $\begin{array}{l}\mathrm{Ce}-141 \\
\mathrm{fCi} / \mathrm{m} 3\end{array}$ & $\begin{array}{l}\mathrm{CE}-144 \\
\mathrm{fCi} / \mathbf{1} 3\end{array}$ \\
\hline $4 / 12-4 / 15$ & 150 & & & & & & & & 0.017 & & \\
\hline $4 / 15-4 / 17$ & 119 & & & & & & & & 0.644 & & \\
\hline $4 / 17-4 / 19$ & 126 & & & & & & & & 0.058 & & \\
\hline $4 / 19-4 / 22$ & 44 & & & & & & & & $0.0 \overline{11}$ & & \\
\hline $4 / 22-4 / 24$ & 143 & & & & & & & & 0.033 & & \\
\hline $4 / 24-4 / 26$ & 131 & 0.026 & & & & & & & 0.046 & & \\
\hline $4 / 2 b-4 / 29$ & 103 & & & & & & & & -0.020 & & \\
\hline $4 / 29-5 / 1 / 91$ & 91 & & & & & & & & -0.017 & & \\
\hline $5 / 1-5 / 3$ & 172 & & & & & & & & -0.017 & & \\
\hline $5 / 3-5 / 6$ & 177 & 0.017 & & & & & & & -0.029 & & \\
\hline $5 / 6-5 / 8$ & 143 & & & & & & & & -0.018 & & \\
\hline $5 / 8-5 / 10$ & 151 & & & & & & & & -0.016 & & \\
\hline $5 / 10-5 / 13$ & 156 & & & & & & & & -0.063 & & \\
\hline $5 / 13-5 / 15$ & 99 & & & & & & & & -0.026 & & \\
\hline $5 / 15-5 / 17$ & 77 & & & 0.04 & & & & & -0.028 & & \\
\hline $5 / 17-5 / 20$ & 71 & & & & & & & & -0.011 & & \\
\hline $5 / 20-5 / 22$ & 131 & & & & & & & & -0.021 & & \\
\hline $5 / 22-5 / 24$ & 135 & & & & & & & & -0.016 & & \\
\hline $5 / 24-5 / 28$ & 83 & & & & & & & & -0.021 & & \\
\hline $5 / 28-5 / 29$ & 69 & & & & & & & & -0.060 & & \\
\hline $5 / 29-5 / 31$ & 69 & & & 0.02 & & & & & -0.021 & & \\
\hline $5 / 31-6 / 3 / 91$ & 125 & & & & & & & & -0.020 & & \\
\hline $6 / 3-6 / 5$ & 159 & & & & & & & & 0.026 & & \\
\hline $6 / 5-6 / 7$ & 124 & & & & & & & & 0.031 & & \\
\hline $6 / 7-6 / 10$ & 90 & & & & & & & & 0.025 & & \\
\hline $6 / 10-6 / 12$ & 154 & & & & & & & & 0.032 & & \\
\hline $6 / 12-6 / 14$ & 116 & & & & & & & & 0.015 & & \\
\hline $6 / 14-6 / 17$ & 107 & 0.018 & & & & & & & -0.013 & & \\
\hline $6 / 17-6 / 19$ & 81 & & & & & & & & -0.019 & & \\
\hline $6 / 17-6 / 21$ & 49 & & & & & & & & -0.015 & & \\
\hline $6 / 21-6 / 24$ & 86 & & & & & & & & -0.018 & & \\
\hline $6 / 24-6 / 26$ & 93 & & & & & & & & 0.040 & & \\
\hline $6 / 26-6 / 28$ & 117 & & & & & & & & 0.032 & & \\
\hline $6 / 28-7 / 1 / 91$ & 50 & & & & & & & & -0.011 & & \\
\hline $7 / 1-7 / 3$ & 79 & & & & & & & & -0.027 & & \\
\hline $7 / 3-7 / 8$ & 83 & 0.007 & & & & & & & 0.015 & & \\
\hline $7 / 8-7 / 10$ & 80 & & & & & & & & 0.039 & & \\
\hline $7 / 10-7 / 12$ & 101 & & & & & & & & 0.032 & & \\
\hline $7 / 12-7 / 15$ & 131 & 0.017 & & & & & & & -0.019 & & \\
\hline $7 / 15-7 / 17$ & 81 & & & & & & & & -0.024 & & \\
\hline $7 / 17-7 / 19$ & 96 & & & & & & & & -0.013 & & \\
\hline $7 / 19-7 / 2 \mathrm{a}$ & 105 & & & & & & & & -0.017 & & \\
\hline $7 / 22-7 / 24$ & 158 & 0.036 & & & & & & & 0.030 & & \\
\hline $7 / 24-7 / 26$ & 124 & & & & & & & & 0.023 & & \\
\hline $7 / 26-7 / 29$ & 64 & & & & & & & & -0.013 & & \\
\hline $7 / 29-7 / 31$ & 35 & & & & & & & & -0.027 & & \\
\hline $7 / 31-8 / 2 / 91$ & 40 & & & & & & & & -0.019 & & \\
\hline $8 / 2-8 / 5$ & 91 & & & & & & & & $-0.01 \overline{8}$ & & \\
\hline $8 / 5-8 / 7$ & 131 & & & & & & & & 0.033 & & \\
\hline $8 / 7-8 / 9$ & 119 & & & & & & & & -0.024 & & \\
\hline $8 / 9-8 / 12$ & 77 & & & & & & & & 0.020 & & \\
\hline $8 / 12-8 / 14$ & 90 & & & & & & & & -0.020 & & \\
\hline $8 / 14-8 / 16$ & 106 & & & & & & & & -0.015 & & \\
\hline
\end{tabular}




\begin{tabular}{|c|c|c|c|c|c|c|c|c|c|c|c|}
\hline Date & $\begin{array}{c}\text { Be }-7 \\
\text { fCi } 1 \text { 两3 }\end{array}$ & $\begin{array}{r}\mathrm{Hz}-2 \mathrm{E} \\
\mathrm{fCi} / \mathrm{m}\end{array}$ & 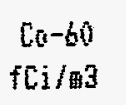 & $\begin{array}{l}\text { Mb-95 } \\
\mathrm{fCi} / \mathbf{6} 3\end{array}$ & $\begin{array}{l}2 r-95 \\
\mathrm{fCi} / \mathrm{n} 3\end{array}$ & $\begin{array}{l}\mathrm{Sib}-103 \\
\mathrm{fCi} / \mathrm{As}\end{array}$ & $\begin{array}{l}\mathrm{Ku}-106 \\
\mathrm{fCi} / \mathbf{4} 3\end{array}$ & $\begin{array}{l}{[5-134} \\
f[j / 1 / 1]\end{array}$ & $\begin{array}{l}\mathrm{C} \leq-137 \\
\mathrm{fCi} / \mathrm{m}\end{array}$ & $\begin{array}{l}{[\mathrm{e}-141} \\
\mathrm{f}[\mathrm{j} / 3\end{array}$ & $\begin{array}{l}{[\mathrm{E}-144} \\
\mathrm{fCi} / \mathrm{3}\end{array}$ \\
\hline $8 / 16-8 / 19$ & 169 & 0.019 & & & & & & & 0.022 & & \\
\hline $8 / 19-8 / 21$ & 122 & & & & & & & & 0.017 & & \\
\hline $8 / 21-8 / 23$ & 122 & & & & & & & & -0.031 & & \\
\hline $8 / 23-8 / 26$ & 71 & & & 0.020 & & & & & -0.019 & & \\
\hline $8 / 26-8 / 28$ & 52 & & & & & & & & $0.0 \mathrm{Eg}$ & & \\
\hline $8 / 28-8 / 30$ & 52 & & & & & & & & -0.028 & & \\
\hline $8 / 30-9 / 3 / 91$ & 121 & 0.013 & & & & & & & 0.011 & & \\
\hline $9 / 3-9 / 4$ & 184 & & & & & & & & -0.054 & & \\
\hline $9 / 4-9 / 6$ & 117 & & & & & & & & -0.019 & & \\
\hline $9 / 6-9 / 9$ & 119 & & & & & & & & -0.021 & & \\
\hline $9 / 9-9 / 11$ & 168 & & & & & & & & 0.032 & & \\
\hline $9 / 11-9 / 13$ & 111 & & & & & & & & 0.032 & & \\
\hline $9 / 13-9 / 16$ & 117 & & & & & & & & 0.037 & & \\
\hline $9 / 16-9 / 18$ & 119 & & & & & & & & 0.084 & & \\
\hline $9 / 18-9 / 20$ & 119 & & & & & & & & 0.021 & & \\
\hline $9 / 20-9 / 23$ & 128 & & & & & & & & 0.016 & & \\
\hline $9 / 23-9 / 25$ & 106 & 0.020 & & & & & & & -0.017 & & \\
\hline $9 / 25-9 / 27$ & 78 & & & & & & & & -0.027 & & \\
\hline $9 / 27-9 / 30$ & 142 & & & & & & & & 0.020 & & \\
\hline $9 / 30-10 / 2 / 91$ & 162 & 0.015 & & & & & & & 0.026 & & \\
\hline $10 / 2-10 / 4$ & 203 & & & & & & & & -0.026 & & \\
\hline $10 / 4-10 / 7$ & 90 & & & & & & & & -0.020 & & \\
\hline $10 / 7-10 / 9$ & 74 & 0.018 & & & & & & & 0.021 & & \\
\hline $10 / 9-10 / 11$ & 185 & & & & & & & & -0.030 & & \\
\hline $10 / 11-10 / 14$ & 144 & & & & & & & & 0.033 & & \\
\hline $10 / 14-10 / 16$ & 138 & & & & & & & & -0.019 & & \\
\hline $10 / 16-10 / 18$ & 173 & & & & & & & & -0.016 & & \\
\hline $10 / 28-10 / 21$ & 140 & & & & & & & & 0.026 & & \\
\hline $10 / 21-10 / 23$ & 126 & & & & 0.02 & & & & 0.050 & & \\
\hline $10 / 23-10 / 25$ & 144 & & & & 0.04 & & & & -0.028 & & \\
\hline $10 / 25-10 / 28$ & 176 & & & & & & & & 0.027 & & \\
\hline $10 / 18-10 / 30$ & 134 & & & & & & & & 0.029 & & \\
\hline $10 / 30-11 / 1 / 91$ & 166 & & & & & & & & 0.051 & & \\
\hline $11 / 1-11 / 4$ & 100 & & & & & & & & -0.013 & & \\
\hline $11 / 4-11 / 6$ & 186 & & & & & & & & 0.019 & & \\
\hline $11 / 6-11 / 8$ & 208 & & & & & & & & -0.051 & & \\
\hline $11 / 8-11 / 11$ & 86 & $0.0 \mathrm{ez}$ & & & & & & & -0.017 & & \\
\hline $11 / 11-11 / 13$ & 212 & & & & & & & & -0.019 & & \\
\hline $11 / 13-11 / 15$ & 113 & & & & & & & & 0.034 & & \\
\hline $11 / 15-11 / 18$ & 133 & & 0.02 & & & & & & 0.034 & & \\
\hline $11 / 18-11 / 20$ & 170 & & & & & & & & -0.026 & & \\
\hline $11 / 20-11 / 22$ & 78 & & & & & & & & -0.026 & & \\
\hline $11 / 22-11 / 25$ & 119 & & & & & & & & -0.016 & & \\
\hline $11 / 25-11 / 27$ & 151 & & & & & & & & -0.022 & & \\
\hline $11 / 27-12 / 1 / 91$ & 137 & & & & & & . & & 0.033 & & \\
\hline $12 / 1-12 / 3$ & 69 & & & & & & & & -0.018 & & \\
\hline $12 / 3-12 / 6$ & 123 & & & & & & & & -0.013 & & \\
\hline $12 / 6-12 / 9$ & 167 & 0.019 & & & & & & & 0.030 & & \\
\hline $12 / 9-12 / 11$ & 107 & & & & & & & & -0.028 & & \\
\hline $12 / 11-12 / 13$ & 115 & & & & & & & & 0.031 & & \\
\hline $12 / 13-12 / 16$ & 142 & & & & & & & & 0.014 & & \\
\hline $12 / 16-12 / 18$ & 160 & & & & & & & & -0.040 & & \\
\hline $15 / 18-15 / 20$ & 143 & & & & & & & & -0.019 & & \\
\hline
\end{tabular}




\begin{tabular}{|c|c|c|c|c|c|c|c|c|c|c|}
\hline & $8 \mathrm{E}^{-}$ & $\begin{array}{r}\mathrm{Hz}-2 \mathrm{2} 2 \\
\mathrm{fCi} / \text { i } 3\end{array}$ & $\begin{array}{r}C n-60 \\
+\mathrm{Ci} / 43\end{array}$ & 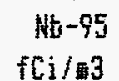 & $\begin{array}{r}2 \mathrm{~T}-95 \mathrm{~s} \\
\mathrm{fCi} / \mathbf{- 1} 3\end{array}$ & 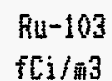 & $\begin{array}{l}\mathrm{Ru}-106 \\
\mathrm{fCS} / \overline{4} 3\end{array}$ & $\begin{array}{l}{[5-134} \\
{[C 1 / 43}\end{array}$ & 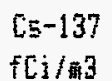 & $\begin{array}{l}\mathrm{CE}-141 \\
\mathrm{fCJ} / \mathrm{J} / 3\end{array}$ \\
\hline
\end{tabular}

\begin{tabular}{|c|c|c|c|c|}
\hline $12 / 20-12 / 23$ & 144 & & & 0.624 \\
\hline $12 / 23-12 / 26$ & 147 & & & 0.009 \\
\hline $12 / 26-12 / 27$ & 96 & & & -0.041 \\
\hline $12 / 27-12 / 30$ & $4 \hat{2}$ & & & -0.021 \\
\hline $12 / 30-1 / 3 / 92$ & 123 & & & -0.013 \\
\hline $1 / 3-1 / 6$ & 48 & & & -0.015 \\
\hline $1 / 6-1 / 8$ & 204 & & & -0.019 \\
\hline $1 / 8-1 / 10$ & 174 & & & 0.034 \\
\hline $1 / 10-1 / 13$ & 123 & & 0.04 & 0.024 \\
\hline $1 / 13-1 / 15$ & 46 & & & -0.019 \\
\hline $1 / 15-1 / 17$ & 170 & & & -0.028 \\
\hline $1 / 17-1 / 20$ & 158 & & & -0.025 \\
\hline $1 / 20-1 / 22$ & 144 & 0.019 & & -0.019 \\
\hline $1 / 22-1 / 24$ & 105 & & & -0.015 \\
\hline $1 / 24-1 / 27$ & 84 & & & -0.019 \\
\hline $1 / 27-1 / 29$ & 127 & & & 0.028 \\
\hline $1 / 29-1 / 31$ & 119 & & & -0.015 \\
\hline $1 / 31-2 / 3 / 92$ & 156 & 0.017 & & 0.013 \\
\hline $2 / 3-2 / 5$ & 175 & & & -0.049 \\
\hline $2 / 5-2 / 7$ & 123 & & & -0.026 \\
\hline $2 / 7-2 / 10$ & 142 & & & -0.020 \\
\hline $2 / 10-2 / 12$ & 123 & & & 0.036 \\
\hline $2 / 12-2 / 14$ & 123 & & & 0.038 \\
\hline $2 / 14-2 / 18$ & 62 & & & 0.027 \\
\hline $2 / 18-2 / 19$ & 15 & & & -0.110 \\
\hline $2 / 19-2 / 21$ & 77 & & & -0.015 \\
\hline $2 / 21-2 / 24$ & 81 & & & 0.021 \\
\hline $2 / 24-2 / 2 b$ & 106 & & & -0.029 \\
\hline $2 / 2 b-2 / 28$ & 76 & & & -0.025 \\
\hline $2 / 28-3 / 2 / 92$ & 107 & & & -0.018 \\
\hline $3 / 2-3 / 4$ & 166 & & & -0.026 \\
\hline $3 / 4-3 / 7$ & 91 & 0.011 & & 0.020 \\
\hline $3 / 9-3 / 11$ & 92 & & & 0.025 \\
\hline $3 / 11-3 / 13$ & 212 & 0.016 & & -0.015 \\
\hline $3 / 13-3 / 16$ & 155 & 0.015 & & 0.018 \\
\hline $3 / 16-3 / 18$ & $18 \overline{9}$ & & & 0.050 \\
\hline $3 / 18-3 / 20$ & 179 & & & 0.060 \\
\hline $3 / 20-3 / 23$ & 124 & & & 0.047 \\
\hline $3 / 23-3 / 25$ & 118 & & & 0.018 \\
\hline $3 / 25-3 / 30$ & 140 & 0.014 & & 0.029 \\
\hline $3 / 30-4 / 1 / 92$ & 98 & & & 0.032 \\
\hline $4 / 1-4 / 3$ & 177 & & & 0.031 \\
\hline $4 / 3-4 / 6$ & 155 & & & 0.029 \\
\hline $4 / 8-4 / 8$ & 164 & & & -0.025 \\
\hline $4 / 8-4 / 10$ & 119 & & & 0.040 \\
\hline $4 / 10-4 / 13$ & 92 & & & -0.026 \\
\hline $4 / 13-4 / 15$ & 234 & 0.054 & & 0.058 \\
\hline $4 / 15-4 / 16$ & 153 & & & 0.105 \\
\hline $4 / 16-4 / 20$ & 120 & & 0.02 & 0.029 \\
\hline $4 / 20-4 / 22$ & 66 & & & 0.031 \\
\hline $4 / 22-4 / 24$ & 155 & & & -0.039 \\
\hline $4 / 24-4 / 27$ & 113 & & & -0.018 \\
\hline $4 / 27-4 / 29$ & 159 & & & -0.023 \\
\hline
\end{tabular}


国

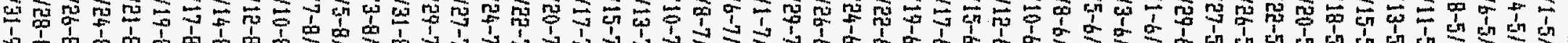

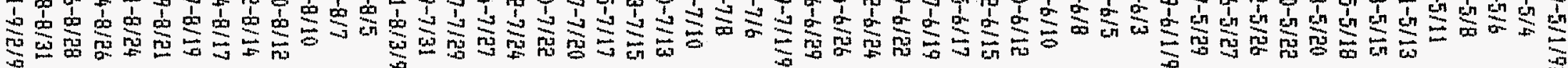

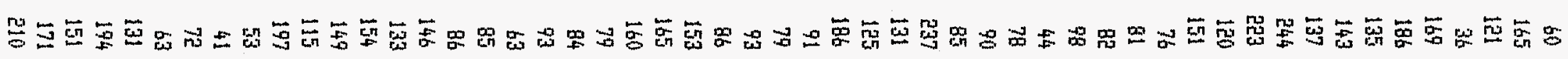

$\stackrel{ }{\mathrm{i}}$

离离密

$\stackrel{8}{9}$

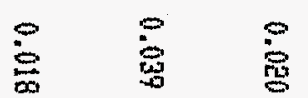

空

总雷

㤩要

营是

ํํำ

욯

옳

总

急品

总蓄

意蓄

营菅

总总

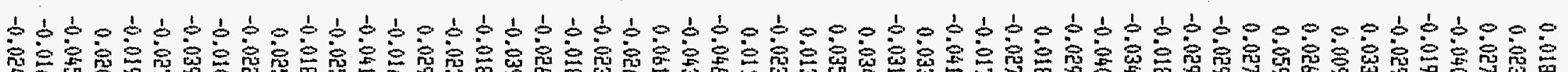
点管 兽螘 查管 
Date Fe-7 Na-22 Co-60 Wb-95 2T-95 Ru-103 Ru-106 C5-134 C5-137 [e-141 Ce-144

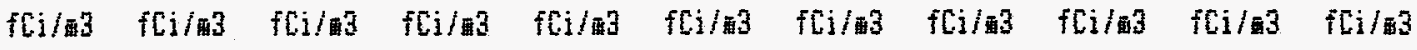

\begin{tabular}{|c|c|c|c|}
\hline $9 / 2-9 / 4$ & 149 & 0.029 & -0.026 \\
\hline $9 / 4-9 / 8$ & 63 & & -0.018 \\
\hline $9 / 8-9 / 9$ & 65 & & -0.055 \\
\hline $9 / 9-9 / 11$ & 94 & & -0.016 \\
\hline $9 / 11-9 / 14$ & 142 & & -0.015 \\
\hline $9 / 14-9 / 16$ & 252 & & -0.026 \\
\hline $9 / 16-7 / 18$ & 140 & & -0.037 \\
\hline $9 / 18-9 / 21$ & 120 & 0.010 & 0.018 \\
\hline $9 / 21-9 / 23$ & 95 & & -0.024 \\
\hline $9 / 23-9 / 25$ & 123 & & -0.047 \\
\hline $9 / 25-9 / 28$ & 52 & & -0.017 \\
\hline $9 / 28-9 / 30$ & 147 & & -0.022 \\
\hline $9 / 30-10 / 2 / 92$ & 193 & & 0.025 \\
\hline $10 / 2-10 / 5$ & 102 & & -0.016 \\
\hline $10 / 5-10 / 7$ & 133 & 0.017 & -0.016 \\
\hline $10 / 7-10 / 9$ & 101 & & -0.020 \\
\hline $10 / 9-10 / 12$ & 172 & & -0.018 \\
\hline $10 / 12-10 / 14$ & 158 & & -0.024 \\
\hline $10 / 14-10 / 16$ & 168 & & -0.027 \\
\hline $10 / 16-10 / 19$ & 176 & 0.021 & -0.018 \\
\hline $10 / 19-10 / 21$ & 178 & & 0.041 \\
\hline $10 / 21-10 / 23$ & 167 & & 0.023 \\
\hline $10 / 23-10 / 26$ & 148 & 0.016 & 0.019 \\
\hline $10 / 2 b-10 / 28$ & 193 & & 0.033 \\
\hline $10 / 28-10 / 30$ & 124 & & 0.028 \\
\hline $10 / 30-11 / 2 / 92$ & 70 & & 0.019 \\
\hline $11 / 2-11 / 4$ & 116 & 0.044 & -0.019 \\
\hline $111 / 4-11 / 6$ & 51 & & -0.023 \\
\hline $11 / 6-11 / 9$ & 62 & & -0.018 \\
\hline $11 / 9-11 / 11$ & 135 & & -0.026 \\
\hline $11 / 11-11 / 13$ & 110 & & 0.019 \\
\hline $11 / 13-11 / 16$ & 195 & & -0.013 \\
\hline $11 / 16-11 / 18$ & 148 & & $-0.02^{2}$ \\
\hline $11 / 18-11 / 20$ & 108 & & 0.030 \\
\hline $11 / 20-11 / 23$ & 71 & & -0.017 \\
\hline $11 / 23-11 / 25$ & 87 & 0.015 & -0.017 \\
\hline $11 / 25-11 / 30$ & 70 & & 0.012 \\
\hline $11 / 30-12 / 2 / 92$ & 142 & & -0.017 \\
\hline $12 / 2-12 / 4$ & 124 & 0.030 & -0.025 \\
\hline $12 / 4-12 / 7$ & 142 & 0.019 & 0.019 \\
\hline $12 / 7-12 / 9$ & 49 & & -0.015 \\
\hline $12 / 9-12 / 11$ & 84 & & -0.020 \\
\hline $12 / 11-12 / 14$ & 45 & & -0.015 \\
\hline $12 / 14-12 / 16$ & 86 & & -0.024 \\
\hline $12 / 16-12 / 18$ & 116 & & $-0.0 \mathrm{e} 7$ \\
\hline $12 / 18-12 / 21$ & 109 & & -0.018 \\
\hline $12 / 21-12 / 23$ & 85 & & -0.025 \\
\hline $12 / 23-12 / 20$ & $17 b$ & & 0.014 \\
\hline $12 / 28-12 / 30$ & 182 & & -0.014 \\
\hline $12 / 30-12 / 31$ & 3 & & -0.048 \\
\hline $12 / 31-1 / 4 / 93$ & 118 & & -0.013 \\
\hline $1 / 4-1 / 6$ & 78 & & $-0,026$ \\
\hline $1 / 6-1 / 8$ & 89 & & -0.026 \\
\hline
\end{tabular}




\begin{tabular}{|c|c|c|c|c|c|c|c|c|c|c|c|}
\hline Doste & $\begin{array}{c}8 \mathrm{E}-7 \\
f[i / m 3\end{array}$ & $\begin{array}{c}\mathrm{Ha}-2 \mathrm{Z} \\
\mathrm{f}\left[\mathrm{Ci} / \mathrm{HI}^{\mathrm{B}} \mathrm{I}\right.\end{array}$ & $\begin{array}{r}C \mathrm{i}-60 \\
\mathrm{fCi} / \mathrm{m}\end{array}$ & $\begin{array}{r}\mathrm{Nt}-95 \mathrm{I} \\
\mathrm{fCi} / \mathrm{t} 3\end{array}$ & $\begin{array}{l}2 t-95 \\
7[j / 63\end{array}$ & $\begin{array}{l}\text { Ru-103 } \\
\mathrm{rCi} / \mathbb{m}^{3}\end{array}$ & 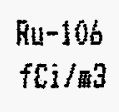 & $\begin{array}{l}{[5-134} \\
f[i / \text { 配 }\end{array}$ & $\begin{array}{l}{[5-13]} \\
f C i / 43\end{array}$ & $\begin{array}{l}\mathrm{CE}-141 \\
\mathrm{fCi} / \mathrm{w} 3\end{array}$ & $\begin{array}{l}\mathrm{Ce}-144 \\
\mathrm{fCi} / \overline{4} 3\end{array}$ \\
\hline $1 / 8-1 / 11$ & 84 & & & & & & & & -0.010 & & \\
\hline $1 / 11-1 / 13$ & E & & & & & & & & -0.022 & & \\
\hline $1 / 13-1 / 15$ & 120 & & & & & & & & -0.023 & & \\
\hline $1 / 15-1 / 18$ & 123 & & & & & & & & $-0.01 z$ & & \\
\hline $1 / 18-1 / 20$ & 28 & & & & & & & & -0.013 & & \\
\hline $1 / 20-1 / 22$ & 379 & & & & & & & & -0.066 & & \\
\hline $1 / 22-1 / 25$ & 92 & & & & & & & & -0.026 & & \\
\hline $1 / 25-1 / 27$ & 138 & 0.026 & & & & & & & -0.020 & & \\
\hline $1 / 27-1 / 29$ & 124 & & & & & & & & -0.042 & & \\
\hline $1 / 29-2 / 1 / 93$ & 202 & 0.030 & & & & & & & -0.018 & & \\
\hline $2 / 1-2 / 3$ & 192 & & & 0.02 & & & & & -0.022 & & \\
\hline $2 / 3-2 / 5$ & 245 & 0.038 & & & & & & & 0.031 & & \\
\hline $2 / 5-2 / 8$ & 117 & & & & & & & & 0.029 & & \\
\hline $2 / 8-2 / 10$ & 74 & & & & & & & & $-0.0 \mathrm{e}$ & & \\
\hline $2 / 10-2 / 12$ & 90 & & & & & & & & -0.040 & & \\
\hline $2 / 12-2 / 16$ & 75 & & & & & & & & -0.014 & & \\
\hline $2 / 1 b-2 / 17$ & $2 h$ & & & & & & & & -0.087 & & \\
\hline $2 / 17-2 / 14$ & 158 & 0.035 & & & & & & & -0.024 & & \\
\hline 2/19-2/22 & 258 & & & & & & & & 0.042 & & \\
\hline $2 / 25-2 / 24$ & 157 & & & & & & & & -0.021 & & \\
\hline $2 / 24-2 / 26$ & 165 & & & & & & & & -0.040 & & \\
\hline $2 / 26-3 / 1 / 93$ & 92 & & & & & & & & -0.018 & & \\
\hline $3 / 1-3 / 3$ & 261 & 0.033 & & & & & & & 0.037 & & \\
\hline $3 / 3-3 / 5$ & 49 & & & & & & & & -0.024 & & \\
\hline $3 / 5-3 / 8$ & 169 & 0.015 & & & & & & & -0.000 & & \\
\hline $3 / 8-3 / 10$ & 132 & & & & & & & & -0.018 & & \\
\hline $3 / 10-3 / 1 \mathrm{E}$ & 155 & 0.018 & & & & & & & 0.015 & & \\
\hline $3 / 12-3 / 15$ & 118 & & & & & & & & 0.015 & & \\
\hline $3 / 15-3 / 17$ & 182 & 0.013 & & & & & & & -0.012 & & \\
\hline $3 / 17-3 / 19$ & 163 & & & & & & & & -0.023 & & \\
\hline $3 / 19-3 / 22$ & 240 & & & & & & & & -0.020 & & \\
\hline $3 / 22-3 / 24$ & 96 & & & & & & & & -0.023 & & \\
\hline $3 / 24-3 / 26$ & 103 & & & & & & & & -0.034 & & \\
\hline $3 / 26-3 / 27$ & 39 & & & & & & & & -0.017 & & \\
\hline $3 / 29-3 / 31$ & 166 & & & & & & & & 0.041 & & \\
\hline $3 / 31-4 / 2 / 93$ & 72 & & & & & & & & 0.036 & & \\
\hline $4 / 2-4 / 5$ & 78 & & & & & & & & -0.028 & & \\
\hline $4 / 5-4 / 7$ & 52 & & & & & & & & -0.016 & & \\
\hline $4 / 7-4 / 8$ & 213 & & & & & & & & -0.083 & & \\
\hline $4 / 8-4 / 12$ & 191 & 0.019 & & & & & & & 0.060 & & \\
\hline $4 / 12-4 / 14$ & 181 & & & & & & & & 0.052 & & \\
\hline $4 / 14-4 / 16$ & 169 & 0.016 & & & & & & & 0.070 & & \\
\hline $4 / 16-4 / 19$ & 198 & 0.017 & & & & & & & 0.027 & & \\
\hline $4 / 19-4 / 21$ & 235 & 0.031 & & & & & & & 0.030 & & \\
\hline $4 / 21-4 / 23$ & 183 & 0.019 & & & & & 0.129 & & 0.030 & & \\
\hline $4 / 23-4 / 26$ & 291 & 0.034 & & & & & & & 0.037 & & \\
\hline $4 / 26-4 / 28$ & 229 & 0.023 & & & & & & & -0.019 & & \\
\hline $4 / 28-4 / 30$ & 248 & 0.039 & & & & & & & $0.05 i$ & & \\
\hline $4 / 30-5 / 3 / 93$ & 206 & 0.024 & & & & & & & -0.021 & & \\
\hline $5 / 3-5 / 5$ & 118 & & & & & & & & 0.031 & & \\
\hline $5 / 5-5 / 7$ & 156 & 0.017 & & & & & & & -0.016 & & \\
\hline $5 / 7-5 / 10$ & 185 & 0.025 & & & & & & & 0.018 & & \\
\hline $5 / 10-5 / 12$ & 205 & 0.031 & & & & & & & 0.032 & & \\
\hline
\end{tabular}




\begin{tabular}{|c|c|c|c|c|c|c|c|c|c|c|c|}
\hline Date & $\begin{array}{c}\mathrm{BE}-7 \\
\{\mathrm{CI} / \mathrm{m} 3\end{array}$ & $\begin{array}{r}\mathrm{Na}-2 \mathrm{Z2} \\
\mathrm{FCi} / \mathrm{m} 3\end{array}$ & $\begin{array}{r}\mathrm{Cr}-60 \\
\mathrm{fCi} / \overline{\mathrm{n}} 3\end{array}$ & 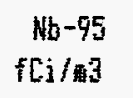 & $\begin{array}{l}2 r-95 \\
\mathrm{f}[\mathrm{i} i / \mathbf{m} 3\end{array}$ & $\begin{array}{l}\mathrm{Ku}-103 \\
\mathrm{fCi} / \mathrm{m} 3\end{array}$ & 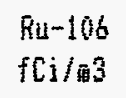 & $\begin{array}{l}\mathrm{Cs}-134 \\
\mathrm{fCi} / 43\end{array}$ & $\begin{array}{l}{[5-137} \\
\{[1 / 6]\end{array}$ & $\begin{array}{l}\mathrm{CE}-141 \\
\mathrm{fCi} / \mathrm{E} 3\end{array}$ & $\begin{array}{l}\mathrm{Ce}-144 \\
\{\mathrm{C} \mathrm{S} / \mathrm{s} 3\end{array}$ \\
\hline $5 / 12-5 / 14$ & 170 & 0.040 & & & & & & & 0.038 & & \\
\hline $5 / 14-5 / 17$ & $18 ?$ & & & & & & & & -0.031 & & \\
\hline $5 / 17-5 / 19$ & 232 & 0.028 & & & & & & & 0.027 & & \\
\hline $5 / 17-5 / 21$ & 121 & & & & & & & & 0.016 & & \\
\hline $5 / 21-5 / 24$ & 162 & 0.024 & & & & & & & -0.020 & & \\
\hline $5 / 24-5 / 26$ & 242 & 0.027 & & & & & & & 0.016 & & \\
\hline $5 / 26-5 / 28$ & 142 & 0.019 & & & & & & & 0.023 & & \\
\hline $5 / 28-6 / 1 / 43$ & 48 & 0.005 & & & & & & & 0.051 & & \\
\hline $6 / 1-6 / 2$ & 76 & & & & & & & & $-0.04 z$ & & \\
\hline $6 / 2-6 / 4$ & 158 & & & & & & & & -0.029 & & \\
\hline $6 / 4-6 / 6$ & 171 & 0.024 & & & & & & & 0.037 & & \\
\hline $6 / 7-6 / 9$ & 153 & & & & & & & & 0.056 & & \\
\hline $6 / 9-6 / 11$ & 175 & & & & & & & & 0.038 & & \\
\hline $6 / 11-6 / 14$ & 154 & & & & & & & & 0.033 & & \\
\hline $6 / 14-6 / 16$ & 127 & & & & & & & & 0.028 & & \\
\hline $6 / 16-6 / 18$ & 162 & & & & & & & & 0.032 & & \\
\hline $6 / 18-6 / 21$ & 166 & & & 0.024 & & & & & -0.018 & & \\
\hline $6 / 21-6 / 23$ & 144 & & & & & & & & -0.026 & & \\
\hline $6 / 23-6 / 25$ & 172 & 0.021 & & & & & & & 0.050 & & \\
\hline $6 / 25-6 / 28$ & 85 & & & & & & & & -0.017 & & \\
\hline $6 / 28-6 / 30$ & 164 & & & & & & & & -0.030 & & \\
\hline $5 / 30-7 / 1 / 93$ & 154 & & & & & & & & -0.041 & & \\
\hline $7 / 1-7 / 6$ & 132 & & & & & & & & -0.027 & & \\
\hline $7 / 6-7 / 7$ & 154 & & & & & & & & 0.048 & & \\
\hline $7 / 7-7 / 9$ & 171 & & & & & & & & 0.024 & & \\
\hline $7 / 9-7 / 12$ & 182 & & & & & & & & 0.038 & & \\
\hline $7 / 12-7 / 14$ & 166 & & & & & & & & 0.047 & & \\
\hline $7 / 14-7 / 16$ & 154 & & & & & & & & 0.031 & & \\
\hline $7 / 16-7 / 19$ & 112 & & & & & & & & -0.021 & & \\
\hline $7 / 19-7 / 21$ & 122 & & & & & & & & 0.026 & & \\
\hline $7 / 21-7 / 23$ & 161 & & & & & & & & 0.032 & & \\
\hline $7 / 23-7 / 26$ & 122 & & & & & & & & 0.015 & & \\
\hline $7 / 2 b-7 / 28$ & 154 & & & & & & & & -0.019 & & \\
\hline $7 / 28-7 / 30$ & 161 & & & & & & & & -0.025 & & \\
\hline $7 / 30-8 / 2 / 93$ & 224 & 0.024 & & & & & & & -0.018 & & \\
\hline $8 / 2-8 / 4$ & 147 & 0.018 & & & & & & & $0.03 \dot{b}$ & & \\
\hline $9 / 4-8 / 6$ & 135 & & & & & & & & -0.027 & & \\
\hline $8 / 6-8 / 7$ & 107 & & & & & & & & -0.020 & & \\
\hline $8 / 9-8 / 11$ & 132 & & & & & & & & -0.027 & & \\
\hline $8 / 11-8 / 13$ & 149 & & & & & & & & 0.037 & & \\
\hline $8 / 13-8 / 16$ & 167 & & & & & & & & 0.039 & $\ldots$ & \\
\hline $8 / 16-8 / 18$ & 195 & & & & & & & & 0.025 & & \\
\hline $8 / 18-8 / 20$ & 147 & & & & & & & & 0.023 & & \\
\hline $8 / 20-8 / 23$ & 140 & 0.014 & & & & & & & 0.040 & & \\
\hline $8 / 23-8 / 25$ & 238 & & & & & & & & -0.014 & & \\
\hline $8 / 25-8 / 27$ & 205 & & & & & & & & 0.106 & & \\
\hline $8 / 27-8 / 30$ & 103 & & & & & & & & -0.018 & & \\
\hline $9 / 30-9 / 1$ & 176 & & & & & & & & 0.040 & & \\
\hline $9 / 1-9 / 7 / 93$ & 100 & 0.008 & & & & & & & 0.019 & & \\
\hline $9 / 7-9 / 8$ & 446 & 0.039 & & & & & & & 0.099 & & \\
\hline $9 / 8-9 / 10$ & 122 & & & & & & & & -0.014 & & \\
\hline $9 / 10-7 / 13$ & 158 & & & & & & & & -0.015 & & \\
\hline $7 / 13-9 / 15$ & 202 & & & & & & & & -0.030 & & \\
\hline
\end{tabular}




\begin{tabular}{|c|c|c|c|c|c|c|c|c|c|c|c|}
\hline Dáte & 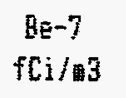 & $\begin{array}{c}1 \mathrm{a}-22 \\
\mathrm{fri} / \mathrm{m}\end{array}$ & $\begin{array}{l}C n-60 \\
5 \mathrm{Cl} / \mathrm{ma}\end{array}$ & 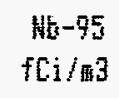 & $\begin{array}{l}2 \mathrm{i}-95 \\
\mathrm{f}[\mathrm{i} / \mathrm{s} / \mathrm{m}\end{array}$ & $\begin{array}{l}\text { Ru-103 } \\
\text { fCi/as }\end{array}$ & $\begin{array}{l}\mathrm{Ru}-106 \\
\mathrm{fCi} / \mathrm{m}^{3} 3\end{array}$ & $\begin{array}{l}{[5-134} \\
f \mathrm{ci} / \mathrm{w}^{2} 3\end{array}$ & $\begin{array}{l}\text { C5-137 } \\
\text { fCi } 1 \text { - } 33\end{array}$ & $\begin{array}{l}\mathrm{Ce}-141 \\
\mathrm{fCi} / \mathrm{E} 3\end{array}$ & $\begin{array}{l}\text { CE-144 } \\
\text { fCi/m }\end{array}$ \\
\hline $9 / 15,9 / 17$ & 103 & & & & & & & & -0.031 & & \\
\hline $9 / 17-9 / 20$ & 115 & & 0.058 & & & & & & -0.018 & & \\
\hline $9 / 20-9 / 22$ & 137 & & & & & & & & -0.016 & & \\
\hline $9 / 22-9 / 24$ & 175 & & & & & & & & $-0.02 ?$ & & \\
\hline $9 / 24-9 / 27$ & 192 & & & & & & & & -0.020 & & \\
\hline $9 / 27-9 / 29$ & 199 & 0.018 & & & & & & & -0.014 & & \\
\hline $9 / 29-10 / 1 / 93$ & 157 & & & & & & & & -0.048 & & \\
\hline $10 / 1-10 / 4$ & 269 & & & & & & & & -0.020 & & \\
\hline $10 / 4-10 / 6$ & 149 & & & & & & & & -0.026 & & \\
\hline $10 / 6-10 / 8$ & 158 & & & & & & & & -0.026 & & \\
\hline $10 / 8-10 / 11$ & 140 & & & & & & & & -0.019 & & \\
\hline $10 / 11-10 / 12$ & 134 & & & & & & & & -0.046 & & \\
\hline $10 / 12-10 / 13$ & 200 & & & & & & & & -0.057 & & \\
\hline $10 / 13-10 / 14$ & 168 & & & & & & & & -0.053 & & \\
\hline $10 / 14-10 / 15$ & 170 & & & & & & & & -0.048 & & \\
\hline $10 / 15-10 / 18$ & 143 & & & & & & & & -0.017 & & \\
\hline $10 / 18-10 / 19$ & $\overline{41}$ & & & & & & & & -0.051 & & \\
\hline $10 / 19-10 / 20$ & 100 & & & & & & & & -0.050 & & \\
\hline $10 / 20-10 / 21$ & 106 & & & & & & & & -0.032 & & \\
\hline $10 / 21-10 / 22$ & 145 & & & & & & & & -0.046 & & \\
\hline $10 / 22-10 / 25$ & 186 & & & & & & & & -0.018 & & \\
\hline $10 / 25-10 / 26$ & 237 & & & & & & & & $-0.04 \dot{b}$ & & \\
\hline $10 / 2 b-10 / 27$ & 185 & & & & & & & & -0.050 & & \\
\hline $10 / 27-10 / 28$ & 201 & & & & & & & & -0.044 & & \\
\hline $10 / 28-10 / 29$ & 310 & & & & & & & & -0.044 & & \\
\hline $10 / 29-11 / 1 / 93$ & 59 & & & & & & & & -0.019 & & \\
\hline $11 / 1-11 / 2$ & 56 & & & & & & & & -0.049 & & \\
\hline $11 / 2-11 / 3$ & 82 & & & & & & & & -0.041 & & \\
\hline $11 / 3-11 / 4$ & 139 & & & & & & & & -0.647 & & \\
\hline $11 / 4-11 / 5$ & 225 & & & & & & & & 0.081 & & \\
\hline $11 / 5-11 / 8$ & 80 & & & & & & & & -0.017 & & \\
\hline $11 / 8-11 / 10$ & 69 & & & & & & & & -0.018 & & \\
\hline $11 / 10-11 / 12$ & 190 & & & & & & & & -0.020 & & \\
\hline $11 / 12-11 / 15$ & 166 & & & & & & & & 0.020 & & \\
\hline $11 / 15-11 / 17$ & 105 & & & & & & & & -0.026 & & \\
\hline $11 / 17-11 / 19$ & 137 & & & & & & & & -0.027 & & \\
\hline :i1/19-11/22 & 162 & & & & & & & & -0.016 & & \\
\hline $11 / 22-11 / 24$ & 274 & 0.050 & & & & & & & 0.021 & & \\
\hline $11 / 24-11 / 29$ & 158 & & & & & & & & 0.024 & & \\
\hline $11 / 29-12 / 1 / 93$ & 238 & & & & & & & & -0.044 & & \\
\hline $12 / 1-12 / 3$ & 125 & & & & & & & & -0.013 & & \\
\hline $12 / 3-12 / 6$ & 107 & 0.014 & & & & & & & -0.013 & & \\
\hline $12 / 6-12 / 8$ & 74 & & & & & & & & -0.015 & & \\
\hline $12 / 9-12 / 10$ & 113 & & & & & & & & 0.023 & & \\
\hline $12 / 10-12 / 13$ & 172 & 0.018 & & & & & & & 0.027 & & \\
\hline $12 / 13-12 / 15$ & 182 & 0.026 & & & & & & & 0.054 & & \\
\hline $12 / 15-12 / 17$ & 44 & & & & & & & & -0.013 & & \\
\hline $12 / 17-12 / 20$ & 89 & & & & & & & & 0.072 & & \\
\hline $12 / 20-12 / 22$ & 5 & & & & & & & & 0.090 & & \\
\hline $12 /[2-1 / 27$ & 61 & & & & & & & & 0.092 & & \\
\hline $12 / 27-12 / 29$ & 185 & & & & & & & & -0.027 & & \\
\hline $12 / 29-12 / 30$ & 128 & & & & & & & & 0.127 & & \\
\hline $12 / 30-1 / 3 / 74$ & 168 & 0.015 & & & & & & & 0.030 & & \\
\hline
\end{tabular}




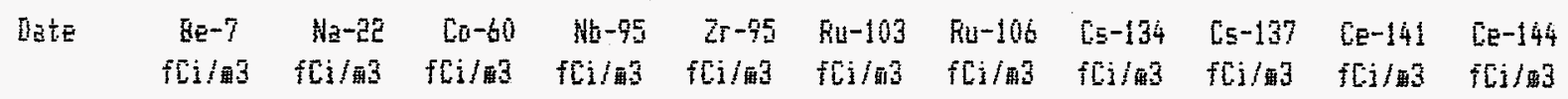

\begin{tabular}{|c|c|c|c|}
\hline $1 / 3-1 / 5$ & 64 & & -0.015 \\
\hline $1 / 5-1 / 7$ & 77 & & 0.033 \\
\hline $1 / 7-1 / 10$ & 135 & & -0.011 \\
\hline $1 / 10-1 / 12$ & 120 & & 0.017 \\
\hline $1 / 12-1 / 14$ & 41 & & -0.019 \\
\hline $1 / 14-1 / 17$ & 175 & 0.019 & -0.013 \\
\hline $1 / 17-1 / 19$ & 160 & & -0.015 \\
\hline $\mid / 19-1 / 21$ & 209 & & -0.017 \\
\hline $1 / 21-1 / 24$ & 183 & & 0.047 \\
\hline $1 / 24-1 / 26$ & 207 & 0.020 & 0.035 \\
\hline $1 / 2 b-1 / \overline{2} 8$ & 95 & & -0.019 \\
\hline $1 / 29-1 / 31$ & 146 & & $-0.0 \mathrm{R} 9$ \\
\hline $1 / 31-2 / 2 / 94$ & 177 & & -0.023 \\
\hline $2 / 2-2 / 4$ & 148 & & -0.018 \\
\hline $2 / 4-2 / 7$ & 170 & 0.017 & 0.015 \\
\hline $2 / 7-2 / 9$ & 144 & 0.012 & 0.015 \\
\hline $2 / 9-2 / 11$ & 44 & & -0.022 \\
\hline $2 / 11-2 / 14$ & $5 \hat{L}$ & & $-0.01 \mathrm{~g}$ \\
\hline $2 / 14-2 / 16$ & 114 & & -0.015 \\
\hline $2 / 16-2 / 18$ & 173 & 0.023 & 0.031 \\
\hline $2 / 18-2 / 22$ & 164 & 0.015 & -0.010 \\
\hline $2 / 22-2 / 23$ & 127 & & -0.036 \\
\hline $2 / 23-2 / 25$ & 104 & & -0.015 \\
\hline $2 / 25-2 / 28$ & 207 & 0.025 & -0.013 \\
\hline $2 / 28-3 / 2 / 94$ & 156 & & 0.023 \\
\hline $3 / 2-3 / 4$ & 89 & & -0.015 \\
\hline $3 / 4-3 / 7$ & 147 & 0.020 & 0.025 \\
\hline $3 / 7-3 / 9$ & 168 & & 0.055 \\
\hline $3 / 9-3 / 11$ & 152 & 0.016 & $-0,015$ \\
\hline $3 / 11-3 / 14$ & 289 & 0.039 & 0.014 \\
\hline $3 / 14-3 / 16$ & 185 & & -0.025 \\
\hline $3 / 16-3 / 18$ & 248 & 0.018 & $0.0 \mathrm{e2}$ \\
\hline $3 / 18-3 / 21$ & 241 & 0.030 & 0.030 \\
\hline $3 / 21-3 / 23$ & 178 & 0.023 & 0.121 \\
\hline $3 / 23-3 / 25$ & 171 & 0.019 & 0.083 \\
\hline $3 / 25-3 / 28$ & 140 & & 0.063 \\
\hline $3 / 28-3 / 30$ & 196 & 0.026 & 0.026 \\
\hline $3 / 30-3 / 31$ & 166 & & -0.041 \\
\hline $3 / 31-4 / 4 / 94$ & 194 & 0.008 & 0.027 \\
\hline $4 / 4-4 / 6$ & 241 & & 0.050 \\
\hline $4 / 6-4 / 8$ & 122 & & $-0.0 \mathrm{e} 2$ \\
\hline $4 / 8-4 / 11$ & 247 & 0.027 & -0.017 \\
\hline $4 / 11-4 / 13$ & 200 & $0.02 \theta$ & 0.022 \\
\hline $4 / 13-4 / 15$ & 257 & 0.035 & 0.024 \\
\hline $4 / 15-4 / 18$ & 202 & 0.026 & -0.015 \\
\hline $4 / 18-4 / 20$ & 314 & 0.037 & 0.095 \\
\hline $4 / 20-4 / 22$ & 201 & & -0.024 \\
\hline $4 / 22-4 / 25$ & 250 & 0.041 & -0.022 \\
\hline $4 / 25-4 / 27$ & 232 & 0.039 & -0.020 \\
\hline $4 / 27-4 / 29$ & 216 & & 0.087 \\
\hline $4 / 29-5 / 2 / 94$ & 279 & 0.030 & 0.020 \\
\hline $5 / 2-5 / 4$ & 115 & & 0.036 \\
\hline $5 / 4-5 / 6$ & 49 & & -0.021 \\
\hline
\end{tabular}




\begin{tabular}{|c|c|c|c|c|c|c|c|c|c|c|}
\hline Date & $\begin{array}{c}\text { Be-7 } \\
f C \mathrm{CH} / \mathrm{A3}\end{array}$ & $\begin{array}{r}\mathrm{Ha}-\mathrm{zE} \\
\mathrm{fCi} / \mathrm{a}\end{array}$ & $\begin{array}{r}50-60 \\
f[i / m 3\end{array}$ & $\begin{array}{r}\mathrm{Hb}-95 \\
\mathrm{fCj} / \text { m }^{3} 3\end{array}$ & $\begin{array}{r}2 \mathrm{r}-75 \\
\mathrm{fCi} / \mathrm{m}\end{array}$ & $\begin{array}{l}5 u-103 \\
5[\mathrm{C} / \mathrm{A} 3\end{array}$ & $\begin{array}{l}\text { Fu-10b } \\
\text { fCi/ } / 3\end{array}$ & $\begin{array}{l}C E-134 \\
\{\mathrm{Ci} / \mathrm{A} 3\end{array}$ & $\begin{array}{l}{[5-137} \\
\mathrm{fC} \mathrm{I} / \mathrm{3} 3\end{array}$ & $\begin{array}{l}{[E-141} \\
{[C j / \text { 雨 } 3}\end{array}$ \\
\hline
\end{tabular}

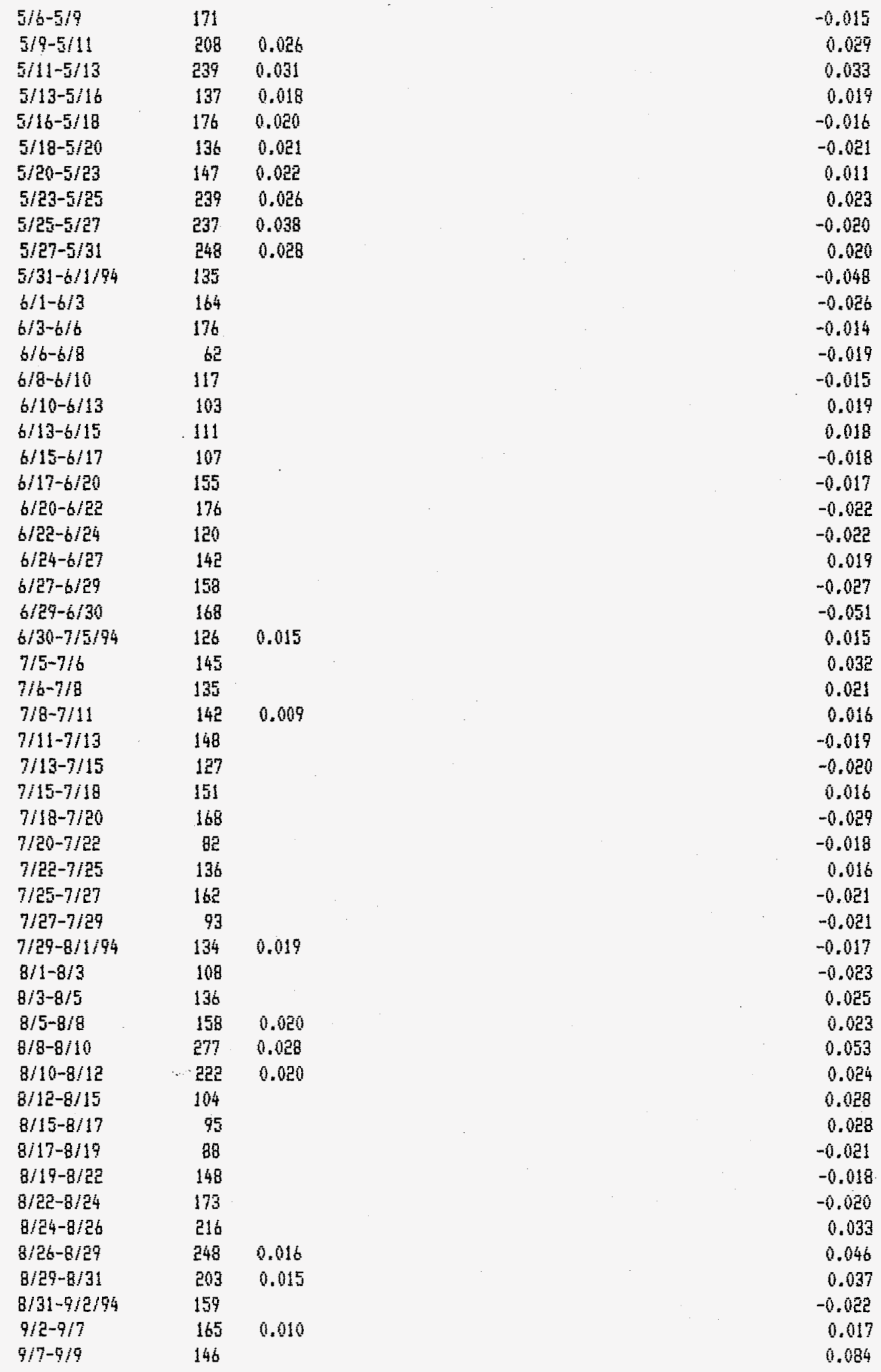




\begin{tabular}{|c|c|c|c|c|c|c|c|c|c|c|c|}
\hline Dete & 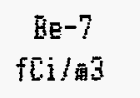 & $\begin{array}{r}\mathrm{Na}-2 \mathrm{2} \\
\mathrm{fCi} / \mathrm{m}\end{array}$ & $\begin{array}{r}\mathrm{Ca}-60 \\
\mathrm{fCi} / \mathbf{m} 3\end{array}$ & $\begin{array}{r}\mathrm{WL}-95 \\
\mathrm{fCi} / \overline{\mathrm{m}} 3\end{array}$ & $\begin{array}{r}2 \mathrm{r}-9 \mathrm{~s} \\
\mathrm{f}[\mathrm{si} / \mathrm{w}\end{array}$ & $\begin{array}{l}6 u-103 \\
6 \mathrm{Cs} / \mathrm{m}\end{array}$ & $\begin{array}{l}\text { Ku- } 10 \mathrm{~b} \\
\mathrm{fCi} / \mathrm{w3}\end{array}$ & $\begin{array}{l}{[5-154} \\
f[\mathrm{i} / / 3\end{array}$ & 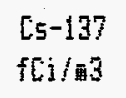 & $\begin{array}{l}{[\mathrm{E}-14 \mathrm{1}} \\
\mathrm{fC \textrm {B }} / \mathrm{B}\end{array}$ & $\begin{array}{l}\text { CE-14 } \\
\text { fCi } / \text { its }\end{array}$ \\
\hline $9 / 9-9 / 12$ & 201 & 0.024 & & & & & & & 0.037 & & \\
\hline $9 / 12-9 / 14$ & 250 & 0.014 & & & & & & & 0.047 & & \\
\hline $4 / 14-9 / 16$ & 269 & & & & & & & & 0.0 .3 & & \\
\hline $9 / 16-9 / 19$ & 130 & & & & & & & & 0.037 & & \\
\hline $9 / 19-9 / 21$ & 108 & 0.023 & & & & & & & -0.020 & & \\
\hline $9 / 21-9 / 23$ & 209 & & 0.02 & & & & & & 0.043 & & \\
\hline $9 / 23-9 / 26$ & 171 & & & & & & & & 0.028 & & \\
\hline $9 / 26-9 / 28$ & 180 & & & & & & & & -0.022 & & \\
\hline $9 / 28-9 / 30$ & 237 & & & & & & & & 0.028 & & \\
\hline $9 / 30-10 / 3 / 94$ & 208 & & & & & & & & 0.019 & & \\
\hline $10 / 3-10 / 5$ & 149 & & 0.02 & & & & & & -0.020 & & \\
\hline $10 / 5-10 / 7$ & 145 & & & & & & & & -0.019 & & \\
\hline $10 / 7-10 / 10$ & 162 & 0.012 & & & & & & & 0.012 & & \\
\hline $10 / 10-10 / 12$ & 187 & & & & & & & & -0.025 & & \\
\hline $10 / 12-10 / 14$ & 143 & & & & & & & & -0.025 & & \\
\hline $10 / 14-10 / 17$ & 209 & & & & & & & & -0.020 & & \\
\hline $10 / 17-10 / 19$ & 277 & 0.017 & & & & & & & 0.016 & & \\
\hline $10 / 19-10 / 24$ & 145 & & & & & & & & -0.013 & & \\
\hline $10 / 24-10 / 26$ & 230 & 0.016 & & & & & & & 0.018 & & \\
\hline $10 / 26-$ & & & & & & & & & & & \\
\hline $10 / 22-10 / 31$ & 140 & 0.014 & & & & & & & 0.017 & & \\
\hline $10 / 31-11 / 2 / 94$ & 125 & & & & & & & & -0.024 & & \\
\hline $11 / 2-11 / 7$ & 186 & & & & & & & & 0.020 & & \\
\hline $11 / 7-$ & & & & & & & & & & & \\
\hline $11 / 9-11 / 11$ & 128 & & & & & & & & 0.021 & & \\
\hline $11 / 11-11 / 14$ & 311 & 0.016 & & & & & & & -0.012 & & \\
\hline $11 / 14-11 / 16$ & 266 & 0.017 & & & & & & & -0.016 & & \\
\hline $11 / 16-11 / 18$ & 116 & & & & & & & & $-0.0 \mathrm{eE}$ & & \\
\hline $11 / 1 a-11 / 21$ & 162 & & & & & & & & -0.017 & & \\
\hline $11 / 21-11 / 23$ & 207 & 0.021 & & & & & & & -0.019 & & \\
\hline $11 / 23-11 / 28$ & 183 & & & & & & & & 0.013 & & \\
\hline $11 / 28-11 / 30$ & 264 & & & & & & & & -0.026 & & \\
\hline $11 / 30-$ & & & & & & & & & & & \\
\hline $12 / 2-12 / 5 / 94$ & 140 & & & & & & & & -0.017 & & \\
\hline $12 / 5-12 / 7$ & 215 & & & & & & & & -0.042 & & \\
\hline $12 / 7-12 / 9$ & 105 & & & & & & & & -0.016 & & \\
\hline $12 / 9-12 / 12$ & 232 & & & & & & & & -0.064 & & \\
\hline $12 / 12-12 / 14$ & 241 & & & & & & & & -0.027 & & \\
\hline $12 / 14-12 / 16$ & 163 & & & & & & & & -0.023 & & \\
\hline $12 / 1 b-12 / 19$ & 96 & & & & & & & & -0.016 & & \\
\hline $12 / 19-12 / 21$ & 74 & & & & & & & & 0.034 & & \\
\hline 12/21-12/22 & $9 b$ & & & & & & & & -0.028 & & \\
\hline $12 / 22-12 / 27$ & 173 & & & & & & & & -0.010 & & \\
\hline $12 / 27-12 / 28$ & 232 & & & & & & & & -0.049 & & \\
\hline $12 / 28-12 / 30$ & 165 & & & & & & & & -0.028 & & \\
\hline $12 / 30-1 / 3 / 95$ & 185 & 0.015 & & & & & & & -0.010 & & \\
\hline $1 / 3-1 / 4$ & 247 & & & & & & & & -0.027 & & \\
\hline $1 / 4-1 / 6$ & 229 & & & & & & & & -0.028 & & \\
\hline $1 / 6-1 / 9$ & 582 & & & & & & & & 0.034 & & \\
\hline $1 / 9-1 / 11$ & 157 & & & & & & & & 0.028 & & \\
\hline $1 / 11-1 / 13$ & 134 & & & & & & & & 0.036 & & \\
\hline $1 / 13-1 / 16$ & 124 & & & & & & & & -0.018 & & \\
\hline $1 / 16-1 / 18$ & 101 & & & & & & & & -0.025 & & \\
\hline
\end{tabular}




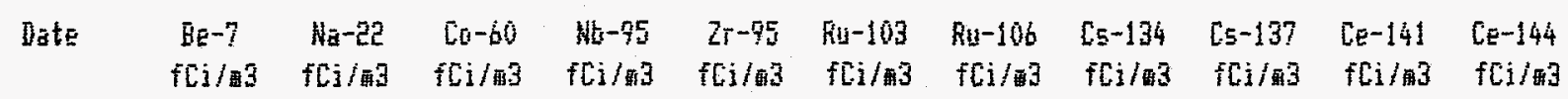

\begin{tabular}{|c|c|c|c|c|}
\hline $1 / 18-1 / 20$ & 71 & & & 0.030 \\
\hline $1 / 20-1 / 23$ & 112 & & & -0.014 \\
\hline $1 / 23-1 / 25$ & 191 & & & -0.020 \\
\hline $1 / 25-1 / 27$ & 208 & & & -0.026 \\
\hline $1 / 27-1 / 30$ & 152 & & & -0.017 \\
\hline $1 / 30-2 / 1 / 95$ & 109 & & & $-0.0 \mathrm{EE}$ \\
\hline $2 / 1-2 / 3$ & 148 & & & $-0.0 \mathrm{es}^{5}$ \\
\hline $2 / 3-2 / 6$ & 215 & & & 0.015 \\
\hline $2 / 6-2 / 8$ & $14 b$ & 0.029 & & -0.023 \\
\hline $2 / 8-2 / 10$ & 212 & & & -0.026 \\
\hline $2 / 10-2 / 13$ & 183 & & & -0.015 \\
\hline $2 / 13-2 / 15$ & 188 & 0.018 & & -0.015 \\
\hline $2 / 15-2 / 17$ & 65 & & & -0.023 \\
\hline $2 / 17-2 / 21$ & 109 & & & -0.009 \\
\hline $2 / 21-2 / 22$ & 68 & & & -0.043 \\
\hline 2/E2-2/24 & 173 & & & -0.026 \\
\hline 2/24-2/27 & 249 & 0.017 & & 0.021 \\
\hline $2 / 27-3 / 1 / 95$ & 189 & 0.030 & 0.040 & 0.040 \\
\hline $3 / 1-3 / 3$ & $11 \overline{2}$ & & & -0.030 \\
\hline $3 / 3-3 / 6$ & 166 & & 0.030 & 0.024 \\
\hline $3 / 6-3 / 6$ & 116 & & & 0.042 \\
\hline $3 / 8-3 / 10$ & 178 & & & 0.030 \\
\hline $3 / 10-3 / 13$ & 326 & & & 0.032 \\
\hline $3 / 13-3 / 15$ & 282 & & & 0.044 \\
\hline $3 / 15-3 / 17$ & 275 & 0.021 & & 0.037 \\
\hline $3 / 17-3 / 20$ & 164 & 0.017 & & 0.030 \\
\hline $3 / 20-3 / 22$ & 229 & 0.016 & & 0.076 \\
\hline $3 / 22-3 / 24$ & 248 & & & 0.111 \\
\hline $3 / 24-3 / 27$ & 192 & 0.026 & & 0.121 \\
\hline $3 / 27-3 / 29$ & 212 & 0.037 & & 0.196 \\
\hline $3 / 29-3 / 31$ & 157 & & & 0.054 \\
\hline $3 / 31-4 / 3 / 95$ & 146 & & & 0.051 \\
\hline $4 / 3-4 / 5$ & 290 & & & 0.085 \\
\hline $4 / 5-4 / 7$ & 229 & 0.027 & & 0.024 \\
\hline $4 / 7-4 / 10$ & 187 & 0.020 & & 0.025 \\
\hline $4 / 10-4 / 12$ & 137 & & & 0.044 \\
\hline $4 / 12-4 / 13$ & 69 & & & -0.023 \\
\hline $4 / 13-4 / 17$ & 235 & 0.030 & & 0.021 \\
\hline $4 / 17-4 / 19$ & 283 & 0.028 & & 0.025 \\
\hline $\begin{array}{l}4 / 19-4 / 21 \\
4 / 21-\end{array}$ & 223 & 0.026 & & 0.035 \\
\hline $4 / 24-4 / 2 b$ & 14.5 & & & 0.022 \\
\hline
\end{tabular}

